\title{
THE RELATIONSHIP BETWEEN MOLECULAR GAS, H I, AND STAR FORMATION IN THE LOW-MASS,
} LOW-METALLICITY MAGELLANIC CLOUDS

\author{
Katherine E. Jameson ${ }^{1}$, Alberto D. Bolatto ${ }^{1}$, Adam K. Leroy ${ }^{2}$, Margaret Meixner ${ }^{3}$, Julia Roman-Duval ${ }^{3}$, \\ Karl Gordon $^{3}$, Annie Hughes ${ }^{4}$, Frank P. Israel ${ }^{5}$, Monica Rubio ${ }^{6}$, Remy Indebetouw ${ }^{7,8}$, Suzanne C. Madden ${ }^{9}$, \\ Caroline Bot $^{10}$, Sacha Hony ${ }^{11}$, Diane Cormier ${ }^{11}$, Eric W. Pellegrini ${ }^{12}$, Maud Galametz ${ }^{13}$, and George Sonneborn ${ }^{14}$ \\ ${ }^{1}$ Astronomy Department and Laboratory for Millimeter-wave Astronomy, University of Maryland, College Park, MD 20742, USA; kjameson@ astro.umd.edu \\ ${ }^{2}$ Department of Astronomy, The Ohio State University, 4051 McPherson Laboratory, 140 West 18th Avenue, Columbus, OH 43210, USA \\ Space Telescope Science Institute, 3700 San Martin Dr., Baltimore, MD 21218, USA \\ 4 Max-Planck-Institut für Astronomie, Königstuhl 17 D-69117 Heidelberg, Germany \\ ${ }^{5}$ Sterrewacht Leiden, Leiden University, P.O. Box 9513, 2300 RA Leiden, The Netherlands \\ ${ }^{6}$ Departamento de Astronoma, Universidad de Chile, Casilla 36-D, Chile
${ }^{7}$ Department of Astronomy, University of Virginia, P.O. Box 400325, Charlottesville, VA 22904, USA \\ ${ }^{8}$ National Radio Astronomy Observatory, 520 Edgemont Road, Charlottesville, VA 22903, USA \\ ${ }^{9}$ Laboratoire AIM, CEA, Université Paris VII, IRFU/Service d'Astrophysique, Bat. 709, F-91191 Gif-sur-Yvette, France \\ ${ }_{11}^{10}$ Observatoire Astronomique de Strasbourg, Université de Strasbourg, CNRS, UMR 7550, 11 rue de l'Université, F-67000 Strasbourg, France \\ ${ }^{11}$ Institut für Theoretische Astrophysik, Zentrum für Astronomie, Universität Heidelberg, Albert-Ueberle-Str. 2, D-69120 Heidelberg, Germany \\ ${ }^{12}$ Department of Physics and Astronomy, University of Toledo, 2801 West Bancroft Street, Toledo, OH 43606, USA \\ ${ }_{13}$ European Southern Observatory, Karl-Schwarzschild-Str. 2, D-85748 Garching-bei-München, Germany \\ ${ }^{14}$ NASA Goddard Space Flight Center, Observational Cosmology Laboratory, Code 665, Greenbelt, MD 20771, USA \\ Received 2015 October 23; revised 2016 April 11; accepted 2016 April 25; published 2016 June 27
}

\begin{abstract}
The Magellanic Clouds provide the only laboratory to study the effects of metallicity and galaxy mass on molecular gas and star formation at high $(\sim 20 \mathrm{pc})$ resolution. We use the dust emission from HERITAGE Herschel data to map the molecular gas in the Magellanic Clouds, avoiding the known biases of CO emission as a tracer of $\mathrm{H}_{2}$. Using our dust-based molecular gas estimates, we find molecular gas depletion times $\left(\tau_{\text {dep }}^{\text {mol }}\right)$ of $\sim 0.4 \mathrm{Gyr}$ in the Large Magellanic Cloud and $\sim 0.6$ in the Small Magellanic Cloud at $1 \mathrm{kpc}$ scales. These depletion times fall within the range found for normal disk galaxies, but are shorter than the average value, which could be due to recent bursts in star formation. We find no evidence for a strong intrinsic dependence of the molecular gas depletion time on metallicity. We study the relationship between the gas and the star formation rate across a range of size scales

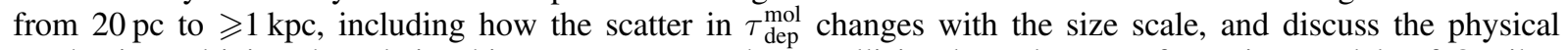
mechanisms driving the relationships. We compare the metallicity-dependent star formation models of Ostriker et al. and Krumholz to our observations and find that they both predict the trend in the data, suggesting that the inclusion of a diffuse neutral medium is important at lower metallicity.
\end{abstract}

Key words: galaxies: dwarf - galaxies: evolution - ISM: clouds - Magellanic Clouds

Supporting material: data behind figures

\section{INTRODUCTION}

Star formation plays a critical role in shaping how galaxies form and evolve. Understanding the molecular gas content of low-mass, low-metallicity galaxies and its relationship to the star formation rate is necessary to understand how the gas mass fractions evolve with redshift (e.g., Tacconi et al. 2010; Genzel et al. 2012) and how the star formation efficiency depends on galaxy mass and metallicity (e.g., Bolatto et al. 2011; Krumholz et al. 2011; Saintonge et al. 2011). Both are critical for understanding the "galaxy mass function" and drivers of the star formation history of the universe.

Our current knowledge of the extragalactic relationship between gas and star formation comes from studies of mostly high-metallicity, high-mass nearby galaxies that use ${ }^{12} \mathrm{CO}$ to trace the molecular gas. The original work to quantitatively compare the star formation rate to the gas density by Schmidt (1959) found a power-law relationship, generally referred to as the "star formation law." More recent studies of the extragalactic star formation law follow the work of Kennicutt $(1989,1998)$, which used primarily disk-averaged measurements of the surface density of total gas $\left(\Sigma_{\mathrm{gas}}=\Sigma_{\mathrm{H}_{2}}+\Sigma_{\mathrm{H}_{\mathrm{I}}}\right)$ and star formation rate $\left(\Sigma_{\mathrm{SFR}}\right)$. They found that the relationship between $\Sigma_{\text {gas }}$ and $\Sigma_{\text {SFR }}$ follows a power-law distribution $\left(\Sigma_{\mathrm{SFR}} \propto \Sigma_{\text {gas }}^{1+p}\right)$. Studies at higher resolution found that the general power-law trend continued, but only within the molecular-dominated regimes and that $\Sigma_{\mathrm{mol}}$ and $\Sigma_{\mathrm{SFR}}$ follow an approximately linear power-law relation (Bigiel et al. 2008, 2011; Schruba et al. 2011; Rahman et al. 2012). At lower gas surface densities, where $\mathrm{H}_{\mathrm{I}}$ dominates the total gas budget, they see a steep fall-off in the relationship. Resolved galaxy studies show that while the total gas continues to be correlated with the star formation rate within galaxies, the molecular gas correlates best with the star formation rate.

Due to the nearly linear power-law slope of the relationship between $\Sigma_{\text {mol }}$ and $\Sigma_{\text {SFR }}$, a convenient way to quantify the relationship is the molecular gas depletion time: $\tau_{\text {dep }}^{\text {mol }}=\Sigma_{\text {mol }} / \Sigma_{\text {SFR }}$. The depletion time can be thought of as the amount of time it would take to deplete the current reservoir of molecular gas given the current star formation rate. Most of the resolved data for samples of galaxies achieve resolutions of several hundred parsecs to $\sim 1 \mathrm{kpc}$ (Bigiel et al. 2008, 2011; Leroy et al. 2008, 2013b; Rahman et al. 2012) and all find similar values for the average molecular gas depletion time of $\tau_{\text {dep }}^{\text {mol }} \sim 2$ Gyr. The weak dependence of $\tau_{\text {dep }}^{\text {mol }}$ on the galactic 
properties and environment (Leroy et al. 2013b) suggest that star formation is a local process based on the conditions within giant molecular clouds (GMCs).

The conclusions from studies of mostly high-mass, highmetallicity disk galaxies may not extend to lower metallicity star-forming dwarf galaxies where the interstellar medium (ISM) is dominated by atomic gas. The lack of metals produce different physical conditions that potentially affect the molecular gas fraction and how star formation proceeds within the galaxy. For example, the galaxies will have lower dust-togas ratios, which results in lower extinctions and higher photodissociation rates. The Large and Small Magellanic Clouds (LMC, SMC) provide ideal laboratories to study the physics of star formation at low mass, $M{ }^{*}$, ${ }_{\text {LMC }}=2 \times 10^{9} M_{\odot}$ and $M^{*}, \mathrm{SMC}=3 \times 10^{8} \quad M_{\odot}$ (Skibba et al. 2012), and low metallicity, $Z_{\mathrm{LMC}} \sim 1 / 2 Z_{\odot}$ (Russell \& Dopita 1992) and $Z_{\mathrm{SMC}} \sim 1 / 5 Z_{\odot}$ (Dufour 1984; Kurt et al. 1999; Pagel 2003), due to their proximity and our ability to achieve high spatial resolution $(\sim 10 \mathrm{pc})$.

Tracing the molecular gas at low metallicity is difficult because $\mathrm{CO}$, the most common tracer of $\mathrm{H}_{2}$, emits weakly and is often undetected. The Magellanic Clouds have been studied extensively in ${ }^{12} \mathrm{CO}$ with the earliest surveys completed using the Columbia $1.2 \mathrm{~m}$ telescope (Cohen et al. 1988; Rubio et al. 1991). The early survey of both Clouds completed by Israel et al. (1993) using the Swedish-ESO Submillimetre Telescope showed the $\mathrm{CO}$ emission to be under-luminous compared to the Milky Way by a factor of $\sim 3$ in the LMC and $\sim 10$ in the SMC. Since then, many large-scale surveys have been completed for the LMC (Fukui et al. 2008; Wong et al. 2011) and SMC (Rubio et al. 1993; Mizuno et al. 2001; Muller et al. 2010). $\mathrm{H}_{2}$ gas is expected to be more prevalent than $\mathrm{CO}$ at low metallicity due to the increased ability of $\mathrm{H}_{2}$ to self-shield against dissociating UV photons compared to $\mathrm{CO}$. Both observations and modeling suggest that $\sim 30 \%-50 \%$ of the $\mathrm{H}_{2}$ in the solar neighborhood resides in a "CO-faint" phase (e.g., Grenier et al. 2005; Wolfire et al. 2010; Planck Collaboration et al. 2011), similar to the estimated fraction of $\mathrm{H}_{2}$ in the LMC (e.g., Roman-Duval et al. 2010; Leroy et al. 2011). Studies of the SMC have found that this phase encompasses $80 \%-90 \%$ of all the $\mathrm{H}_{2}$ (Israel 1997; Pak et al. 1998; Leroy et al. 2007, 2011; Bolatto et al. 2011), likely dominating the molecular reservoir available for star formation.

Using dust emission to estimate the molecular gas in lowmetallicity systems avoids the biases of $\mathrm{CO}$ and can trace "COfaint" molecular gas. This method of tracing the molecular gas using dust emission in the Magellanic Clouds was first applied by Israel (1997) using IRAS data, and later by Leroy et al. (2007, 2009) in the SMC and Bernard et al. (2008) in the LMC using Spitzer data. Bolatto et al. (2011) further refined the methodology and created a map of $\mathrm{H}_{2}$ in the SMC using dust continuum emission from Spitzer and studied the spatial correlation between the atomic gas, molecular gas, and star formation rate. When using the dust-based molecular gas estimate, they found that $\tau_{\text {dep }}^{\text {mol }}$ is consistent with the values seen in more massive disk galaxies. Combining the dust-based molecular gas estimate with the atomic gas traced by $\mathrm{H}_{\mathrm{I}}$ showed that the analytic star formation models of Krumholz et al. (2009) and Ostriker et al. (2010) predicted the trend in the data.

In this work we produce an estimate of the molecular gas using dust emission traced by Herschel in the LMC and SMC.
While the SMC is lower metallicity, the geometry is poorly constrained and it shows clear signs of disturbance from interaction with the LMC and the Milky Way, which makes it problematic for comparisons against models created for galactic disks. We adopt a higher inclination angle for the SMC than was used in Bolatto et al. (2011) to explore how that affects the results. The LMC is nearly face-on with a wellconstrained inclination angle and has a clear disk morphology, which minimizes the uncertainty in the analysis.

We compare the new dust-based molecular gas estimates and atomic gas to the star formation rate in both galaxies and to the existing studies of large disk galaxies. In Sections 2 and 3 we outline the observations and how we convert them to physical quantities. Section 4 presents the main results of this study, focusing on the relationship between molecular gas and star formation, and the effect of scale. We discuss the implications of the results and compare the observations to star formation model predictions in Section 5. Finally, we summarize the conclusions from this study of the LMC and SMC in Section 6.

\section{OBSERVATIONS}

\subsection{Herschel Data}

The far-IR images come from the Herschel Inventory of the Agents of Galaxy Evolution in the Magellanic Clouds key project (HERITAGE; Meixner et al. 2013). HERITAGE mapped both the LMC and SMC at 100, 160, 250, 350, and $500 \mu \mathrm{m}$ with the Spectral and Photometric Imaging Receiver (SPIRE), and the Photodetector Array Camera and Spectrometer (PACS) instruments. Information on the details of the data calibration, reduction, and uncertainty can be found in Meixner et al. (2013).

For this work, we apply further background subtraction. First, we remove the foreground Milky Way cirrus emission. Following Gordon et al. (2014) (based on Bot et al. 2004), we estimate the foreground cirrus emission by using the relationship between IR dust emission and H I from Desert et al. (1990) and scaling the integrated $\mathrm{H}_{\mathrm{I}}$ intensity map over the velocities of the Milky Way emission in the direction of the LMC by applying the conversion factors $1.073,1.848,1.202$, and 0.620 (MJy sr ${ }^{-1} / 10^{2-} \mathrm{cm}^{-2}$ ) for $100 \mu \mathrm{m}, 160 \mu \mathrm{m}, 250 \mu \mathrm{m}$, and $350 \mu \mathrm{m}$ images, respectively. The median estimated cirrus emission was 5.7, 9.9, 6.4, and $3.3 \mathrm{MJy} \mathrm{sr}^{-1}$ for the 100,160 , 250 , and $350 \mu \mathrm{m}$ images.

Second, we set the images to comparable zero-points: the outskirts of the PACS images were set to the COBE and IRAS data emission levels while the outskirts of the SPIRE images were set to zero due to the lack of similar large-scale coverage at the longer wavelengths. After subtracting the cirrus emission, we chose six regions in the outskirts of the LMC with no emission in the Herschel or $\mathrm{H}_{\mathrm{I}}$ images, fit a plane to the median values of the regions, and subtract the plane. The cirrus subtraction and background subtraction had primarily minor effects on the images, with the final image values being lower by $7 \%, 9 \%, 2 \%$, and $2 \%$ on average for the 100,160 , 250 , and $350 \mu \mathrm{m}$ images in regions with a signal-to-noise ratio $(\mathrm{S} / \mathrm{N})>3$.

\subsection{H I Data}

The neutral atomic gas data come from $21 \mathrm{~cm}$ line observations of $\mathrm{HI}$. We use the LMC H I map from Kim et al. (2003) and the SMC H I map from Stanimirović et al. 
(1999), both combine Australian Telescope Compact Array (ATCA) and Parkes $64 \mathrm{~m}$ radio telescope data. The interferometric ATCA data set the map resolution at $1^{\prime}(r \sim 20 \mathrm{pc}$ in the SMC and $r \sim 15 \mathrm{pc}$ in the LMC), but the data are sensitive to all size scales due to the combination of interferometric and single-dish data.

The observed brightness temperature of the $21 \mathrm{~cm}$ line emission is converted to $\mathrm{HI}$ column density $\left(N_{\mathrm{HI}}\right)$ assuming optically thin emission using

$$
N_{\mathrm{H}}=1.823 \times 10^{18} \frac{\mathrm{cm}^{-2}}{\mathrm{~K} \mathrm{~km} \mathrm{~s}^{-1}} \int T_{B}(v) d v .
$$

We find rms column densities of $8.0 \times 10^{19} \mathrm{~cm}^{-2}$ in the LMC map and $5.0 \times 10^{19} \mathrm{~cm}^{-2}$ in the SMC map. We convert column density to surface mass density $\left(\Sigma_{\mathrm{HI}}\right)$ using

$$
\Sigma_{\mathrm{HI}}=1.4 \cos i\left(8.0 \times 10^{-21} \frac{M_{\odot} \mathrm{pc}^{-1}}{\mathrm{~cm}^{-2}}\right) N_{\mathrm{H} \mathrm{I}},
$$

where the factor of 1.4 accounts for $\mathrm{He}$ and $i$ is the inclination angle.

While the assumption of optically thin $\mathrm{H}$ I emission is likely appropriate throughout much of the galaxies, there are regions with optically thick emission, which would cause $N_{\mathrm{H}}$ to be underestimated. While a statistical correction for $\mathrm{H}_{\text {I }}$ optical depth in the SMC exists (Stanimirović et al. 1999), none exists for the LMC. Additionally, nearby surveys of $\mathrm{H}_{\text {I }}$ (i.e., THINGS; Walter et al. 2008) make no optical depth corrections. We chose not to make any optical depth corrections to the $\mathrm{H}$ I maps as the statistical corrections in the SMC are generally small (increases the total $\mathrm{H}$ I mass by $10 \%$; Stanimirović et al. 1999) and an accurate optical depth correction would require assuming a spin temperature.

\subsection{CO Data}

We use integrated ${ }^{12} \mathrm{CO}(1-0)$ intensity maps from the $4 \mathrm{~m}$ NANTEN radio telescope (half power beam width of $2 ! 6$ at $115 \mathrm{GHz}$ ) for the LMC (Fukui et al. 2008) and SMC (Mizuno et al. 2001). The LMC and SMC velocity integrated maps have typical $3 \sigma$ noise of $\sim 1.2 \mathrm{~K} \mathrm{~km} \mathrm{~s}^{-1}$ and $\sim 0.45 \mathrm{~K} \mathrm{~km} \mathrm{~s}^{-1}$, respectively. For the LMC, there is also the higher resolution and sensitivity Magellanic Mopra Assessment (MAGMA) Survey, which used the $22 \mathrm{~m}$ Mopra telescope of the Australia Telescope National Facility to follow-up the NANTEN survey with $40^{\prime \prime}$ angular resolution and $1 \sigma$ sensitivity of $0.2 \mathrm{~K} \mathrm{~km} \mathrm{~s}^{-1}$ (Wong et al. 2011). However, the MAGMA survey is not complete as they only mapped regions with detected $\mathrm{CO}$ in the NANTEN map. Because the CO maps are only used to identify molecular regions and do not affect the final resolution of our molecular gas maps, we use the higher coverage NANTEN maps in our molecular gas mapping process and then, in the LMC, we compare the final dust-based molecular gas maps to the higher resolution MAGMA data.

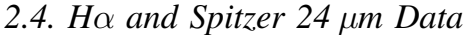

We combine images of $\mathrm{H} \alpha$ and $24 \mu \mathrm{m}$ dust emission to trace recent star formation. For the LMC we use the calibrated, continuum-subtracted $\mathrm{H} \alpha$ map from the Southern $\mathrm{H} \alpha$ Sky Survey Atlas (Gaustad et al. 2001) at 0!'8 resolution. We correct the $\mathrm{H} \alpha$ maps for the line-of-sight Milky Way extinction using $A_{V}(\mathrm{LMC})=0.2 \mathrm{mag}$ and $A_{V}(\mathrm{SMC})=0.1 \mathrm{mag}$ (Schlafly \& Finkbeiner 2011). We found background emission outside the

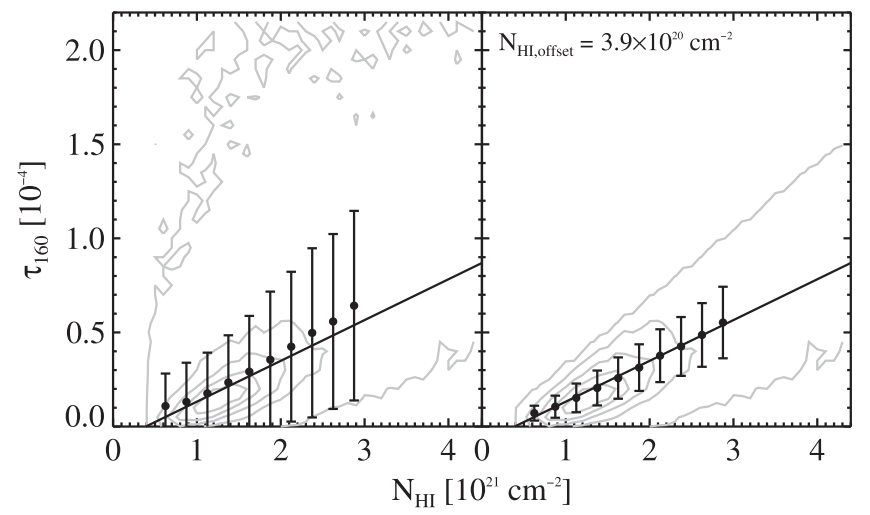

Figure 1. Both plots show the relationship between $N_{\mathrm{HI}}$ and $\tau_{160}$ (from the BEMBB dust modeling) in the LMC with the right-hand plot showing the best representation of the relationship between $N_{\mathrm{HI}}$ and $\tau_{160}$ as the lines of sight with molecular gas have been removed. The contour levels correspond to the full extent of the distribution, $20 \%, 40 \%, 60 \%$, and $80 \%$ of the maximum density of points. The black points show the medians in $2.5 \times 10^{20} \mathrm{~cm}^{-2} N_{\mathrm{H} \mathrm{I}}$ bins with error bars showing $1 \sigma$ of the distribution of measurements within the bin. The plots show the two-stage iteration used to fit the offset in the distribution: the left plot has points near bright $\mathrm{CO}$ emission masked and the right plot has masked points near bright $\mathrm{CO}$ and points with estimated $N_{\text {mol }}>0.5 N_{\mathrm{HI}}$ based on the first iteration. The typical error on $\tau_{160}$ is $\sim 1 \times 10^{-5}$ in regions with predominately $\mathrm{H}_{\mathrm{I}}$ gas, which is similar to the $1 \sigma$ spread in the distribution in the bins. This suggests that the correlation between $N_{\mathrm{H} \mathrm{I}}$ and $\tau_{160}$ is intrinsically very tight and approximately linear, showing that the dust is a good tracer of the gas. We find the offset in $N_{\mathrm{HI}}$ from the fit to the medians in the second iteration. The $N_{\mathrm{H}}$ offset is $\sim 5 \times 10^{20} \mathrm{~cm}^{-2}$ throughout most of the LMC (see Appendix B for further details).

LMC, on the order of $10 \%$ of the total flux observed in the main part of the galaxy, likely from the diffuse Milky Way $\mathrm{H} \alpha$ emission. We apply additional background subtraction by removing a polynomial fit to the regions outside the galaxy. In the SMC, we use the continuum-subtracted $\mathrm{H} \alpha$ map from the Magellanic Cloud Emission Line Survey (MCELS; Smith \& MCELS Team 1999) at 2"!3 resolution. For both the SMC and LMC we use the Multiband Imaging Photometer $24 \mu \mathrm{m}$ map from the Spitzer Survey "Surveying the Agents of Galaxy Evolution" (SAGE; Meixner et al. 2006; Gordon et al. 2011).

\subsection{Distances and Inclination Angles}

To convert observational measurements to surface mass density $(\Sigma)$, we need both the distance to the galaxy and inclination angle $(i)$. For the LMC, we use an inclination angle of $i=35^{\circ}$, which is the approximate intermediate value of the three fits to stellar proper motions and line-of-sight velocity measurements in van der Marel \& Kallivayalil (2014), which range from $i=26^{\circ} .2 \pm 5^{\circ} .9$ to $i=39^{\circ} .6 \pm 4.5$, and is consistent with their previous work that found $i=34.7 \pm 6.2$ (van der Marel \& Cioni 2001). We assume that the inclination of the stellar disk is comparable to the gas disk given the disk-like morphology of the LMC. While Kim et al. (1998) fit an inclination angle to the $\mathrm{H}_{\mathrm{I}}$ kinematics, they found it was unreliable and much higher than the morphological fit $\left(i=22^{\circ}\right.$ $\pm 6^{\circ}$ ). Ultimately, Kim et al. (1998) adopted the inclination angle found from the stellar dynamics.

The inclination of the SMC is poorly constrained due to its irregular morphology. Recent work by Scowcroft et al. (2016) shows that assuming a disk with an inclination angle inaccurately represents the detailed morphology of the SMC. However, comparing the SMC to the LMC and studies of other 


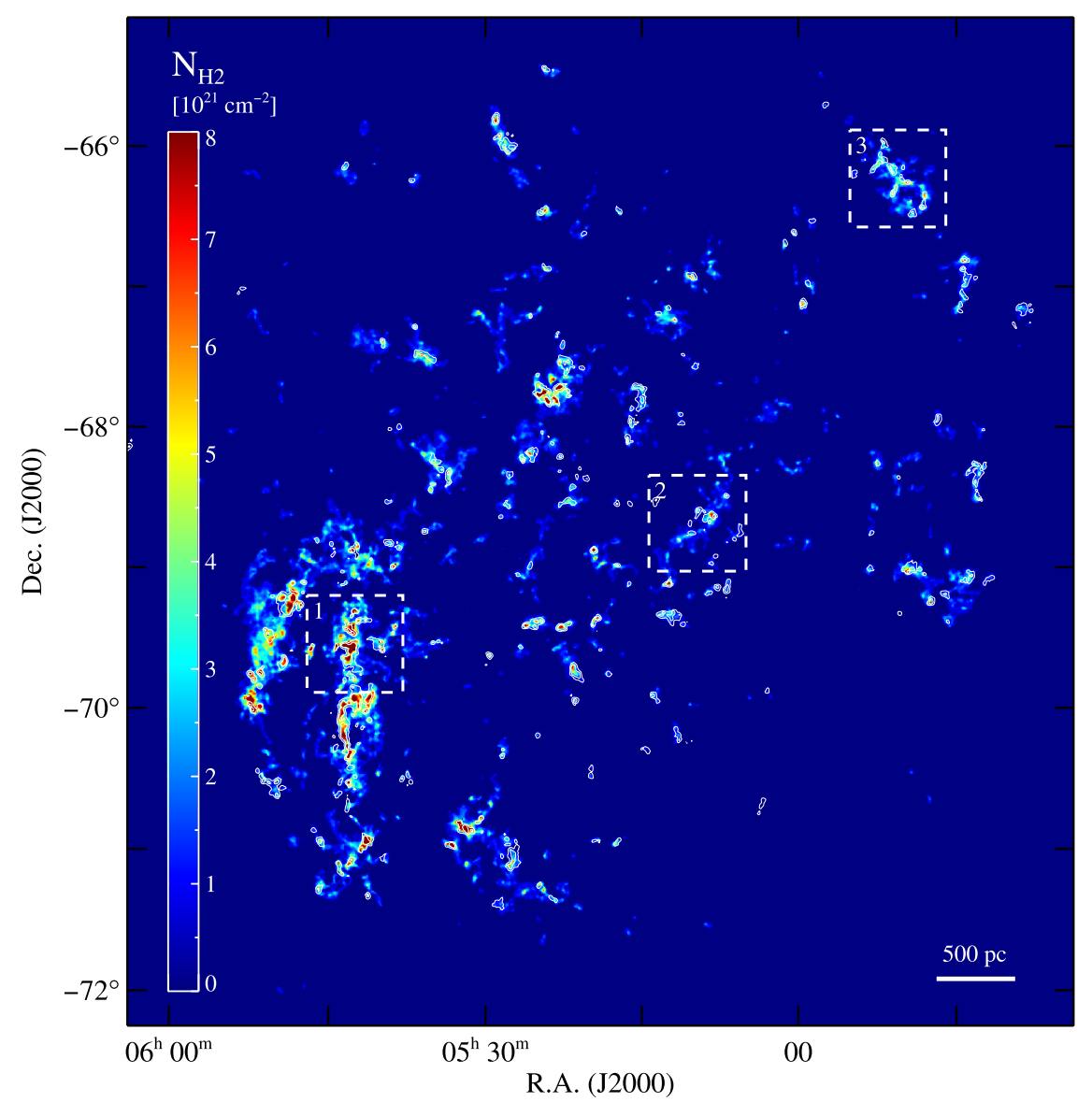

Figure 2. $\mathrm{H}_{2}$ column density $\left(N_{\mathrm{H}_{2}}\right)$ map of the LMC at $\sim 5$ pc resolution $\left(\theta=20^{\prime \prime}, 1\right.$ beam per pixel sampling) produced by modeling the dust continuum emission from Herschel 100, 160, 250, and $350 \mu \mathrm{m}$ observations from HERITAGE (Meixner et al. 2013) using a modified blackbody. The white contours show the $1.2 \mathrm{~K} \mathrm{~km} \mathrm{~s}^{-1}(3 \sigma)$ and $5 \mathrm{~K} \mathrm{~km} \mathrm{~s}^{-1}$ levels of the MAGMA DR3 CO map $\left(\theta=40^{\prime \prime}\right)$, which covered regions with prior CO detection. Assuming a Galactic conversion factor of $X_{\mathrm{CO}}=2 \times 10^{20} \mathrm{~cm}^{-2}\left(\mathrm{~K} \mathrm{~km} \mathrm{~s}^{-1}\right)^{-1}$, the contour levels correspond to column densities of $2.4 \times 10^{20} \mathrm{~cm}^{-2}$ and $1 \times 10^{21} \mathrm{~cm}^{-2}$. The dashed white boxes indicate the three regions in Figure 4. There is excellent agreement between the dust-based molecular gas map and the CO map even though the CO is not directly used to produce the map.

(The data used to create this figure are available.)

galaxies requires knowing the mass surface densities and adopting the simple model of an inclined disk. Bolatto et al. (2011) adopted $i=40^{\circ} \pm 20^{\circ}$ based on the analysis of the $\mathrm{H} \mathrm{I}$ rotation curve by Stanimirović et al. (2004). The recent estimate of the SMC inclination based on three-dimensional structure traced by cepheid variable stars found $i=74^{\circ} \pm 9^{\circ}$ (Haschke et al. 2012), which is consistent with the previous studies using cepheids (Caldwell \& Coulson 1986; Groenewegen 2000). While cepheids, as old stars, may not trace the gaseous disk, a new analysis of the $\mathrm{H}$ I rotation also indicates a higher possible inclination of $i \approx 60^{\circ}-70^{\circ}$ (P. Teuben 2016, private communication). A higher inclination angle scales the surface mass densities to lower values. We adopt $i=70^{\circ}$ for the inclination of the SMC and compare to the previous results in Bolatto et al. (2011) to determine how the higher inclination angle affects the results.

\section{METHODOLOGY}

\subsection{Estimating Molecular Gas from Infrared Dust Emission}

We combine the dust emission with a self-consistently estimated gas-to-dust ratio to estimate the total amount of gas. By removing the atomic gas, we are left with an estimate of the amount of molecular gas. The benefit of this method, particularly at low metallicity, is its ability to trace $\mathrm{H}_{2}$ where $\mathrm{CO}$ has photo-dissociated. This method is based on the previous work by Israel (1997) and Leroy et al. (2011) in both Magellanic Clouds, Dame et al. (2001) in the Milky Way, Bernard et al. (2008) in the LMC, and Leroy et al. $(2007,2009)$ and Bolatto et al. (2011) in the SMC, all of which have demonstrated that dust is a reliable tracer of the molecular gas. In Figure 1, we show that the optical depth of the dust correlates well with $N_{\mathrm{H}}$, which represents the majority of the gas, and the $1 \sigma$ scatter in the distribution is comparable to the uncertainty of $\tau_{160}$ of $\sim 1 \times 10^{-5}$, suggesting there is an intrinsically tight relationship. The variation in the relationship between $N_{\mathrm{H} \text { I }}$ and $\tau_{160}$ that is observed in nearby clouds (see below) could contribute to the observed scatter. The dust is also well correlated with the molecular gas traced by ${ }^{12} \mathrm{CO}$, which is shown for the SMC in Figure 4 in Lee et al. (2015a) using the HERITAGE and MAGMA data. We summarize the specific steps in our methodology, which closely follow the methodology by Leroy et al. (2009) for the SMC, but with improvements allowed by the increased IR coverage and resolution from Herschel. 
Following Leroy et al. (2009) and Bolatto et al. (2011), we model the dust emission in order to obtain the optical depth of the dust emission at $160 \mu \mathrm{m}\left(\tau_{160}\right)$. We use the results from two different dust emission fitting techniques for the LMC, one presented in this paper and another from Gordon et al. (2014), both based on the assumption of modified blackbody emission, $S_{\nu} \propto \nu^{\beta} B_{\nu}\left(T_{d}\right)$. We describe the fitting techniques in more detail in Appendix A. For the SMC, we only produce one molecular gas map using the modeling results from Gordon et al. (2014) since Bolatto et al. (2011) produced a molecular gas map using a fixed $\beta$ simple modified blackbody model and a similar methodology. The Gordon et al. (2014) dust modeling may produce a more accurate measure of $T_{d}$ since it allows $\beta$ to vary while reducing the amount of degeneracy between $T_{d}$ and $\beta$ (Dupac et al. 2003; Shetty et al. 2009) by accounting for the correlated errors between the Herschel bands.

While the dust temperature along the line-of sight throughout the Magellanic Clouds likely has a distribution of temperatures (Bernard et al. 2008; Galliano et al. 2011; Galametz et al. 2013), the assumption of a single dust temperature on the small spatial scales we cover $(\sim 20 \mathrm{pc})$ is reasonable since temperature mixing is restricted. Leroy et al. (2011) ran both simple modified blackbody fits and more complex dust models from Draine \& $\mathrm{Li}$ (2007) to find $\tau_{160}$ using the Spitzer data for the LMC and SMC, and found that both produced similar results. A future follow-up study of Gordon et al. (2014) will run more complex dust modeling of the HERITAGE Herschel data.

This study focuses on using dust emission as a means to estimate the amount of molecular gas, which does not require a measurement of the dust mass. By only using $\tau_{160}$ we avoid making any assumptions about the conversion to dust mass, which would introduce a further layer of uncertainty. We define our effective gas-to-dust $\left(\delta_{\mathrm{GDR}}\right)$ ratio in terms of $\tau_{160}$,

$$
\delta_{\mathrm{GDR}}=\Sigma_{\mathrm{HI}} / \tau_{160}
$$

such that any proportionality constant between the IR intensity and $\tau_{160}$ will be incorporated into $\delta_{\mathrm{GDR}}$ and not affect our final results.

We expect, in principle, that the relationship between $N_{\mathrm{H} \mathrm{I}}$ and $\tau_{160}$ should go through the origin, but our measurements show indications of an offset (see Figure 1). We regionally fit and then remove the offset and find that the relationship has a positive and roughly constant offset in $N_{\mathrm{H} \text { I }}$ in both the LMC $\left(N_{\mathrm{H}} \sim 4 \times 10^{20} \mathrm{~cm}^{-2}\right)$ and $\operatorname{SMC}\left(N_{\mathrm{H}} \sim 1.5 \times 10^{21} \mathrm{~cm}^{-2}\right)$. A similar offset was observed by Leroy et al. (2011), Bolatto et al. (2011), and Roman-Duval et al. (2014). As opposed to Bolatto et al. (2011), we remove the offset to avoid overestimates when creating maps of the gas-to-dust ratios, which would result in higher estimates of the total amount of gas. This offset could be due to a layer of $\mathrm{H}$ I gas with little to no dust, it could be due to the issues with background subtraction with the Herschel images (particularly in the LMC where the HERITAGE maps to not extend much past the main part of the galaxy), or some combination of the two effects. Another possibility is that the relationship between $N_{\mathrm{H} \text { I }}$ and $\tau_{160}$ is nonlinear and the slope (gas-to-dust ratio) decreases at low $N_{\mathrm{H}}$, which we explore as part of the systematic uncertainty estimation (see Sections 3.1.1 and 4.1.2). Determining the true nature of the offset is beyond the scope of this work, but warrants further investigation. We subtract the offset in $N_{\mathrm{H} \text { I }}$ from the $\mathrm{H}_{\mathrm{I}}$ map and use the offset-subtracted map for the rest of the analysis. For further discussion on the offset subtraction see Appendix B.

Steps to produce molecular gas map:

1. Model the dust emission in the Herschel images to obtain $\tau_{160}$ (see Appendix A for more details).

2. Fit the $\mathrm{H}_{\mathrm{I}}$ offset in the $N_{\mathrm{H} \text { I }}$ versus $\tau_{160}$ distribution regionally (see Appendix B for more details).

3. Produce first iteration map of the spatially varying effective gas-to-dust ratio $\left(\delta_{\mathrm{GDR}}\right)$ at $500 \mathrm{pc}$ scales determined from the diffuse regions $\left(\Sigma_{\text {gas }}=\Sigma_{\mathrm{H}_{\mathrm{I}}}\right)$

a. Compute $\delta_{\mathrm{GDR}}$ for each pixel.

b. Mask all pixels that likely have molecular gas: all regions within $2^{\prime}$ of bright $\mathrm{CO}$ emission $\left(I_{\mathrm{CO}}>3 \sigma\right)$.

c. Use averaging of nearest neighbors to iteratively fill in the masked (molecular) regions in the map.

d. Convolve map with symmetric Gaussian with $\mathrm{FWHM}=500 \mathrm{pc}$.

4. Estimate $\Sigma_{\text {mol }}$ using the first iteration of the smoothed effective $\delta_{\mathrm{GDR}}$ :

$$
\Sigma_{\mathrm{mol}}=\left(\delta_{\mathrm{GDR}} \Sigma_{\mathrm{dust}}\right)-\Sigma_{\mathrm{HI}} .
$$

5. Produce second iteration of map of spatially varying $\delta_{\mathrm{GDR}}$ smoothed to $500 \mathrm{pc}$. Same as step 4 with the modification that both regions within $2^{\prime}$ of bright $\mathrm{CO}$ emission $\left(I_{\mathrm{CO}}>3 \sigma\right)$ and points that have estimated $\Sigma_{\text {mol }}>0.5 \Sigma_{\mathrm{H} \mathrm{I}}$ are masked.

6. Produce final map of $\Sigma_{\text {mol }}$ map using the second iteration of the smoothed $\delta_{\mathrm{GDR}}$ map.

The final steps in producing the molecular gas maps remove unphysical artifacts. First, we remove small regions of estimated $\mathrm{H}_{2}$ that are likely spurious by masking pixels that have positive molecular gas in less than $50 \%$ of the pixels surrounding them within a $4^{\prime} \times 4^{\prime}$ box $(12 \times 12$ pixels in the modified blackbody map from this work and $4 \times 4$ pixels in the maps from Gordon et al. (2014); $60 \times 60 \mathrm{pc}$ in the LMC and $\sim 70 \times 70 \mathrm{pc}$ in the SMC). Generally, this removes emission smaller than $\sim 2^{\prime}$ ( $r \sim 30 \mathrm{pc}$ in the LMC and $r \sim 35 \mathrm{pc}$ in the SMC)-two times the beam size of the lower resolution $\mathrm{H}$ I data - and regions of negative values (from underestimated total gas). Second, we median-filter the map over 3 pixels $\left(\sim 1^{\prime}\right.$ in the LMC map from this work) to smooth out the $\Sigma_{\text {mol }}$ map and remove spikes that are unphysical and below the resolution of the $\mathrm{H}_{\mathrm{I}}$ map, largely due to the residual striping from the HERITAGE PACS images (Meixner et al. 2013).

There are a few caveats to this methodology that can potentially bias our molecular gas estimate. In addition to tracing the molecular gas (including any "CO-faint" component), our methodology may also trace optically thick and/or cold $\mathrm{H}_{\mathrm{I}}$ gas that emits disproportionately to the optically thin H I. Stanimirović et al. (1999) takes a statistical approach and estimates the optical depth correction in the SMC based on column density using the absorption line measurements from Dickey et al. (2000) and finds the correction only changes the total $\mathrm{H}_{\mathrm{I}}$ mass by $\sim 10 \%$. Lee et al. (2015b) takes a similar approach to estimate an optical depth correction in the Milky Way and finds that the correction only increases the mass of $\mathrm{H}$ I in the Perseus molecular cloud by $\sim 10 \%$. Braun (2012) attempted to measure the $\mathrm{H}_{\mathrm{I}}$ optical depth from the flattening of the line profile in M31, M33, and the LMC, and found nonnegligible optical depth corrections for high column densities $\left(22<\log \mathrm{N}_{\mathrm{H}}<23\right)$ in compact $(\sim 100 \mathrm{pc})$ regions, which increases the total $\mathrm{HI}_{\mathrm{I}}$ mass by $\sim 30 \%$. The Braun (2012) 
Table 1

Total Molecular Gas Mass Estimates for the LMC and SMC

\begin{tabular}{|c|c|c|c|c|}
\hline & Data & Dust Fitting $^{\mathrm{a}}$ & Method & $M_{\mathrm{mol}}\left[10^{7} M_{\odot}\right]^{\mathrm{b}}$ \\
\hline \multicolumn{5}{|c|}{ LMC } \\
\hline 1 & Herschel $100-350 \mu \mathrm{m}$ & $\mathrm{MBB}, \beta=1.8$ & $\delta_{\mathrm{GDR}} \operatorname{map}^{\mathrm{c}}$ & 9.9 \\
\hline 2 & Herschel $100-500 \mu \mathrm{m}$ & BEMBB, $0.8<\beta<2.5$ & $\delta_{\mathrm{GDR}} \operatorname{map}^{\mathrm{c}}$ & 6.3 \\
\hline 3 & Herschel $100-350 \mu \mathrm{m}$ & $\mathrm{MBB}, \beta=1.5$ & $\delta_{\mathrm{GDR}} \mathrm{map}^{\mathrm{c}}$ & 6.8 \\
\hline 4 & Herschel $100-350 \mu \mathrm{m}$ & $\mathrm{MBB}, \beta=2.0$ & $\delta_{\mathrm{GDR}} \mathrm{map}^{\mathrm{c}}$ & 10.1 \\
\hline 5 & Herschel $100-500 \mu \mathrm{m}$ & BEMBB, $0.8<\beta<2.5$ & $\delta_{\mathrm{GDR}} \operatorname{map}^{\mathrm{c}}$, no $\mathrm{H}_{\mathrm{I}}$ offset & 13.4 \\
\hline 6 & Herschel $100-500 \mu \mathrm{m}$ & BEMBB, $0.8<\beta<2.5$ & $\mathrm{GDR}=540^{\mathrm{d}}$ & 3.9 \\
\hline 7 & Herschel $100-350 \mu \mathrm{m}$ & $\mathrm{MBB}, \beta=1.8$ & $\delta_{\mathrm{GDR}} \operatorname{map}^{\mathrm{c}}, \delta_{\mathrm{GDR}, \mathrm{dense}}=0.5 \delta_{\mathrm{GDR}, \text { map }}$ & 4.5 \\
\hline 8 & Herschel $100-500 \mu \mathrm{m}$ & BEMBB, $0.8<\beta<2.5$ & $\delta_{\mathrm{GDR}} \mathrm{map}^{\mathrm{c}}, \delta_{\mathrm{GDR}, \mathrm{dense}}=0.5 \delta_{\mathrm{GDR}, \mathrm{map}}$ & 4.0 \\
\hline \multicolumn{5}{|c|}{ - } \\
\hline 9 & Herschel $100-500 \mu \mathrm{m}$ & BEMBB, $0.8<\beta<2.5$ & $\delta_{\mathrm{GDR}} \operatorname{map}^{\mathrm{c}}$ & 2.0 \\
\hline
\end{tabular}

Notes.

${ }^{\mathrm{a}} \mathrm{MBB}=$ modified blackbody, $\mathrm{BEMBB}=$ broken emissivity modified blackbody.

${ }^{\mathrm{b}}$ Assuming $d_{\mathrm{LMC}}=50 \mathrm{kpc}$ and $d_{\mathrm{SMC}}=62 \mathrm{kpc}$.

${ }^{\mathrm{c}}$ Map of spatially varying $\delta_{\mathrm{GDR}}$, see Section 3.1.

${ }^{\mathrm{d}}$ Does not include factor of 1.36 contribution from helium.

estimate relies on the assumption of Gaussian line profiles to look for flattening of the $\mathrm{H}_{\mathrm{I}}$ line due to optical depth, which is a difficult measurement in the low $\mathrm{S} / \mathrm{N}$ data. McKee et al. (2015) find $\sim 30 \%$ to be the appropriate $\mathrm{H}_{\mathrm{I}}$ optical depth correction for the solar neighborhood based on the average correction factors found using absorption line measurements in the plane of the Milky Way. Fukui et al. (2015), on the other hand, find more extreme opacity correction factors, as high as a factor of $\sim 2$ in the plane of the Milky Way using a relationship between $N_{\mathrm{HI}}$ and the optical depth at $353 \mathrm{GHz}$ from Planck. The possible $\mathrm{H}_{\mathrm{I}}$ opacity corrections coming from a variety of methods and data show that the factors are uncertain.

The manner in which the optical depth correction will affect our molecular gas estimates is complex. It can increase the $\mathrm{H}_{\mathrm{I}}$ column density in the regions used to estimate the gas-to-dust ratio, leading to an increase in the total gas estimated in the molecular regions, and/or in the molecular regions, resulting in a decrease in the amount of molecular gas. We choose to use the H I statistical opacity corrections from Stanimirović et al. (1999) and Lee et al. (2015b) to explore how correcting for optical depth effects our methodology in Section 4.1.3.

We note that, in Perseus where the structure of the molecular cloud is resolved, Lee et al. (2015b) compares their map of $\mathrm{H}_{\mathrm{I}}$ with the statistical optical depth correction to their inferred "CO-faint" gas, observing that the structures are not spatially coincident (see Figure 4 and Lee et al. 2015b). This suggests that the "CO-faint" gas cannot be explained by optically thick H I alone. Additionally, Lee et al. (2015b) comment that their opacity corrected H I map does not show the the sharp peaks seen in maps from Braun (2012).

Our methodology also relies on the assumption that the gasto-dust ratio in the diffuse, atomic gas is the same in the molecular regions; we only measure the relationship between gas and dust in the atomic phase. There is observational evidence that the gas-to-dust ratio may vary from the diffuse to the dense gas in the Magellanic Clouds (Bot et al. 2004; Roman-Duval et al. 2014). In the Milky Way, Planck results show an factor of 2 increase in the far-IR dust optical depth per unit column density $\left(\tau_{250} / N_{\mathrm{H}}\right)$ from the diffuse to the dense gas (Planck Collaboration et al. 2011), which would could indicate a lower gas-to-dust ratio in the dense gas. Both optically thick
$\mathrm{HI}$ and a decrease in the gas-to-dust ratio from the diffuse to the dense gas would mimic the effect of molecular gas and would result in our methodology overestimating the amount of molecular gas. We explore how these factors could affect our measurement of $\mathrm{H}_{2}$ in our systematic uncertainty estimate.

\subsubsection{Map Sensitivity and Uncertainty}

We use a Monte Carlo method to estimate the uncertainty in our molecular gas maps and determine the sensitivity levels. For the maps produced with the dust fitting from this work, we select three sub-regions (shown in Figure 2) with different levels of molecular gas (high, moderate, and low). We add normally distributed noise with an amplitude equal to the uncertainty to each of the Herschel bands and fit $T_{d}$ for a fixed $\beta$ for each sub-region and then calculate $\tau_{160}$. For the dust modeling results from Gordon et al. (2014), we add normally distributed noise to the $\tau_{160}$ maps with an amplitude equal to the uncertainty estimates from Gordon et al. (2014). Finally, we add noise to the H I map and create new $\Sigma_{\text {mol }}$ maps. The process is repeated 100 times for each of the different maps. We use the distribution of $\Sigma_{\mathrm{mol}}$ for each pixel from the Monte Carlo realizations to estimate a realistic uncertainty. The sensitivity of the maps is estimated by finding the lowest $\Sigma_{\text {mol }}$ that is consistently recovered at $\geqslant 2 \sigma$.

We know that the systematic uncertainty from the methodology will dominate the uncertainty in our molecular gas maps (Leroy et al. 2009; Bolatto et al. 2011). To estimate the level of systematic uncertainty, we see how changes to various aspect of the mapping methodology affect the estimated total molecular mass $M_{\text {mol }}$ (which includes the factor of 1.4 to account for $\mathrm{He}$ ). We explore the effects of different assumptions in the dust modeling and determination of the gas-to-dust ratio, and produces map that:

1. change the value of $\beta$ in our dust modeling and re-run the fitting with $\beta=1.5$ and $\beta=2.0$;

2. do not remove an $\mathrm{H}_{\mathrm{I}}$ offset, which explores the idea that the relationship between $N_{\mathrm{HI}}$ and $\tau_{160}$ may not be linear at low column densities; 
Table 2

Global Properties

\begin{tabular}{lcc}
\hline \hline Property & LMC & SMC \\
\hline$M_{\text {mol }}^{\text {dust }}$ & $6.3_{-3.2}^{+6.3} \times 10^{7} M_{\odot}$ & $2.0_{-1.0}^{+2.0} \times 10^{7} M_{\odot}$ \\
$L_{\mathrm{CO}}$ & $7 \times 10^{6} \mathrm{~K} \mathrm{~km} \mathrm{~s}^{-1 \mathrm{a}}$ & $1.7 \times 10^{5} \mathrm{Km} \mathrm{s}^{-1 \mathrm{~b}}$ \\
$M_{\mathrm{H} \mathrm{I}}$ & $4.8 \times 10^{8} M_{\odot}{ }^{\mathrm{c}}$ & $3.8 \times 10^{8} M_{\odot}{ }^{\mathrm{d}}$ \\
$M_{*}$ & $2 \times 10^{9} M_{\odot}^{\mathrm{e}}$ & $3 \times 10^{8} M_{\odot}^{\mathrm{f}}$ \\
$\mathrm{SFR}^{\mathrm{d}}$ & $0.20 M_{\odot} \mathrm{yr}^{-1}$ & $0.033 M_{\odot} \mathrm{yr}^{-1}$ \\
$\tau_{\text {dep }}^{\text {mol }}$ & $0.37_{-0.19}^{+0.37} \mathrm{Gyr}$ & $0.61_{-0.31}^{+0.61} \mathrm{Gyr}$ \\
\hline
\end{tabular}

Notes.

${ }^{\mathrm{a}}$ Fukui et al. (2008), no sensitivity cuts.

b Mizuno et al. (2001), no sensitivity cuts.

${ }^{\mathrm{c}}$ Staveley-Smith et al. (2003).

d Stanimirović et al. (1999).

e Skibba et al. (2012).

${ }^{\mathrm{f}}$ Assuming $A_{V}(\mathrm{LMC})=0.2 \mathrm{mag}$ and $A_{V}(\mathrm{SMC})=0.1 \mathrm{mag}$.

3. apply a single gas-to-dust ratio using the high and low values from Roman-Duval et al. (2014) (as opposed to using the map of $\delta_{\mathrm{GDR}}$ );

4. scale the $\delta_{\mathrm{GDR}}$ map down by a factor of 2 in the molecular regions to account for a possible change in the gas-to-dust ratio from the diffuse to the dense gas, where we define the dense gas as regions in the map that are likely to have molecular gas (step 5 in Section 3.1);

5. apply a single gas-to-dust ratio for the diffuse gas and a lower value for the dense gas using the values from Roman-Duval et al. (2014), where the dense gas value is applied to regions with bright $\mathrm{CO}$ emission (as in RomanDuval et al. 2014).

For the versions of the maps where we use gas-to-dust ratios found in Roman-Duval et al. (2014), we use the maps of $\Sigma_{\text {dust }}$ in place of $\tau_{160}$. We use the range in $M_{\text {mol values to estimate the }}$ amount of systematic uncertainty in our molecular gas estimate.

\subsubsection{Estimating $\mathrm{H}_{2}$ from $\mathrm{CO}$}

For the purposes of this work, we want to compare the amount of $\mathrm{H}_{2}$ traced by detected, bright ${ }^{12} \mathrm{CO}$ emission to the molecular gas traced by the dust emission. To convert the $\mathrm{CO}$ intensity $\left(I_{\mathrm{CO}}\right)$ into column density of mass, we use the following equations:

$$
\begin{aligned}
& N\left(\mathrm{H}_{2}\right)=X_{\mathrm{CO}} I_{\mathrm{CO}} \\
& M_{\mathrm{mol}}=\alpha_{\mathrm{CO}} L_{\mathrm{CO}},
\end{aligned}
$$

where proportionality constants appropriate for Galactic gas are $X_{\mathrm{CO}}=2 \times 10^{20} \mathrm{~cm}^{-2}\left(\mathrm{~K} \mathrm{~km} \mathrm{~s}^{-1}\right)^{-1}$ and $\alpha_{\mathrm{CO}}=4.3 M_{\odot}(\mathrm{K} \mathrm{km}$ $\left.\mathrm{s}^{-1} \mathrm{pc}^{2}\right)^{-1}, I_{\mathrm{CO}}$ is the integrated intensity of the ${ }^{12} \mathrm{CO}$ $J=1 \rightarrow 0$ transition (in $\mathrm{K} \mathrm{km} \mathrm{s}^{-1}$ ), and $L_{\mathrm{CO}}$ is the luminosity of the same transition (in $\mathrm{K} \mathrm{km} \mathrm{s}^{-1} \mathrm{pc}^{2}$ ). On small spatial scales and in CO-bright regions, using the Galactic values is a good approximation (Bolatto et al. 2008).

\subsection{Tracing Recent Star Formation}

We use $\mathrm{H} \alpha$, locally corrected for extinction using $24 \mu \mathrm{m}$ emission, to trace the star formation rate surface density $\left(\Sigma_{\mathrm{SFR}}\right)$. Following Bolatto et al. (2011), we use the star formation rate calibration by Calzetti et al. (2007) to convert $\mathrm{H} \alpha$ and $24 \mu \mathrm{m}$ luminosities:

$$
\begin{aligned}
\operatorname{SFR}\left(M_{\odot} \mathrm{yr}^{-1}\right)= & 5.3 \times 10^{-42}[L(\mathrm{H} \alpha) \\
& +(0.031 \pm 0.006) L(24 \mu \mathrm{m})]
\end{aligned}
$$

where luminosities are in erg $\mathrm{s}^{-1}$ and $L(24 \mu \mathrm{m})$ is expressed as $\nu L(\nu)$. The average contribution from $24 \mu \mathrm{m}$ to the total star formation rate is $\sim 20 \%$ in the LMC and $\sim 10 \%$ in the SMC. A significant fraction $(\sim 40 \%)$ of the $\mathrm{H} \alpha$ emission in both the LMC and SMC is diffuse. We include all of the $\mathrm{H} \alpha$ emission in this analysis since Pellegrini et al. (2012) showed that all of the ionizing photons could have originated from $\mathrm{H}$ in regions from massive stars (see Appendix $\mathrm{C}$ for further discussion). The rms background value of the star formation rate map is $1 \times 10^{-4} M_{\odot} \mathrm{yr}^{-1} \mathrm{kpc}^{-2}$ in the LMC and $4 \times 10^{-4} M_{\odot} \mathrm{yr}^{-1} \mathrm{kpc}^{-2}$ in the SMC.

This conversion to star formation rate assumes an underlying broken power-law Kroupa initial mass function (IMF) and was calibrated against Paschen- $\alpha$ emission for individual starforming regions. Ideally, $\mathrm{H} \alpha$ and $24 \mu \mathrm{m}$ emission would only be used for size scales that fully sample the IMF and sustain star formation for $>10 \mathrm{Myr}$; for smaller scales, pre-main sequence stars are more appropriate and a better indicator of the current star formation rate. Hony et al. (2015) found that the star formation rate from pre-main sequence stars matches that from $\mathrm{H} \alpha$ at scales of $\sim 150 \mathrm{pc}$ in the N66 region in the SMC. Our highest resolution of $\sim 20 \mathrm{pc}$ resolves $\mathrm{H}$ II regions, and the mapping of the star formation rate on these scales is questionable. Nonetheless, we apply the star formation rate conversion even to our highest resolution data to allow us to compare to other studies and investigate the relationships in terms of a physical quantity, although it is important to keep these limitations in mind when interpreting the results.

\subsection{Convolving to Lower Resolutions}

To produce the lower resolution molecular gas maps, we first convolve the maps from the Herschel beam to a Gaussian with FWHM of $30^{\prime \prime}$ for the $\beta=1.8$ map (appropriate for the $350 \mu \mathrm{m}$ image resolution) and $40^{\prime \prime}$ for the BEMBB map (appropriate for the $500 \mu \mathrm{m}$ image resolution) using the kernels from Aniano et al. (2011). We then produce the range of lower resolution maps (from $20 \mathrm{pc}$ to $\sim 1 \mathrm{kpc}$ ) by convolving the images of $\Sigma_{\mathrm{SFR}}, \Sigma_{\mathrm{mol}}$, and $\Sigma_{\mathrm{H} \text { I }}$ with a Gaussian kernel with FWHM $=\sqrt{\left(r^{2}-r_{0}^{2}\right)}$, where $r$ is the desired resolution and $r_{0}$ is the starting resolution of the image. The images are then resampled to have approximately independent pixels (one pixel per resolution element). To mitigate edge effects from the convolution, we remove the outer two pixels (two beams) for all resolution images of the LMC. In the SMC, we remove two outer pixels for $r \leqslant 600 \mathrm{pc}$ and remove one pixel from the edges for $r \geqslant 700 \mathrm{pc}$ due to the small size of the images.

\section{RESULTS}

\subsection{Molecular Gas in the Magellanic Clouds}

We find molecular gas fractions that are comparable to the Milky Way in the LMC (17\%), but much lower in the SMC (3\%). These molecular gas fractions come from our new estimates of the total molecular gas mass: we find a total molecular gas mass (including $\mathrm{He}$ ) in the LMC of $M_{\mathrm{LMC}}^{\mathrm{mol}}=6.3_{-3.2}^{+6.3} \times 10^{7} \quad M_{\odot}$ and $M_{\mathrm{SMC}}^{\mathrm{mol}}=1.3_{-0.65}^{+1.3} \times 10^{7} M_{\odot}$ 


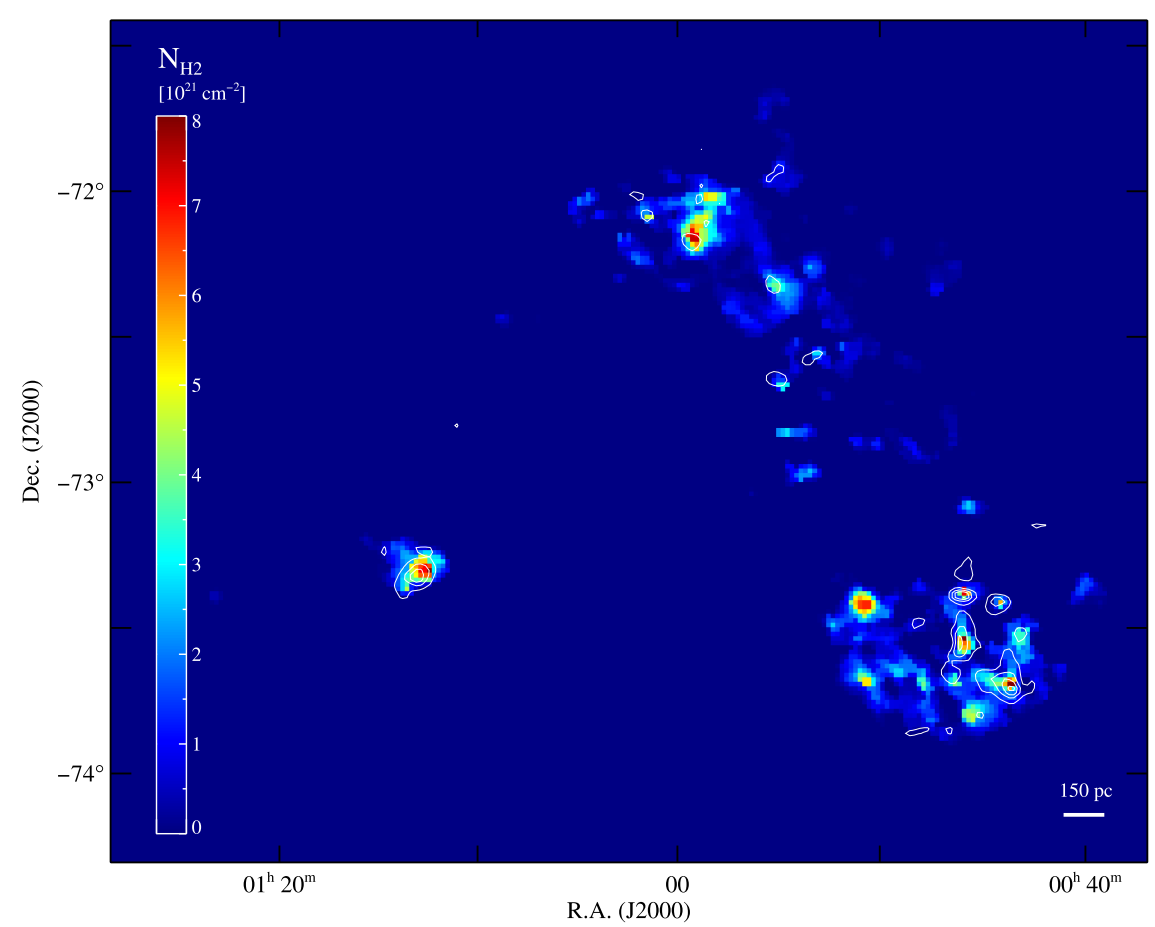

Figure 3. $\mathrm{H}_{2}$ column density $\left(N_{\mathrm{H}_{2}}\right)$ map of the SMC at $\sim 10 \mathrm{pc}$ resolution $\left(\theta=40^{\prime \prime}, 1\right.$ beam per pixel sampling) produced by modeling the dust continuum emission from Herschel 100, 160, 250, 350, and $500 \mu \mathrm{m}$ observations from HERITAGE (Meixner et al. 2013) using the BEMBB dust modeling results from Gordon et al. (2014). The white contours show the $0.45(3 \sigma), 1,1.5$, and $2 \mathrm{~K} \mathrm{~km} \mathrm{~s}^{-1}$ levels of the NANTEN CO map $\left(\theta=1^{\prime}\right)$.

(The data used to create this figure are available.)

in the SMC. These values are the sums (with no cuts) of our fiducial molecular gas maps that use the broken emissivity modified blackbody (BEMBB) dust modeling results from Gordon et al. (2014) with a spatially varying $\delta_{\mathrm{GDR}}$ and include a factor of 2 systematic uncertainty. Table 1 shows the results from our exploration of varying the map making methodology to estimate the systematic uncertainty combined with estimates of the molecular gas mass from the literature. In Table 2 we list the integrated properties of both galaxies. The molecular gas maps are sensitive to $\Sigma_{\text {mol }} \sim 15 M_{\odot} \mathrm{pc}^{-2}\left(\sim 7 \times 10^{20} \mathrm{~cm}^{-2}\right)$ based on the Monte Carlo estimates, which is comparable to the sensitivity of the SMC map from Bolatto et al. (2011). Our molecular gas fraction in the SMC is lower than previous estimates (Leroy et al. 2007; Bolatto et al. 2011), but is consistent with the factor of $\sim 2$ estimate of systematic uncertainty for all of the estimates (see Appendix D for further discussion).

The fiducial molecular gas maps $(\beta=1.8$, broken emissivity modified blackbody (BEMBB) dust modeling) were produced using maps of the effective dust-to-gas ratio $\left(\delta_{\mathrm{GDR}}\right)$ that had average values for $N_{\mathrm{H}} / \tau_{160}$ of $1.8 \pm 0.6 \times 10^{25} \mathrm{~cm}^{-2}$ (LMC $\beta=1.8), 1.3 \pm 0.3 \times 10^{25} \mathrm{~cm}^{-2}$ (LMC BEMBB), and $4.8 \pm$ $0.9 \times 10^{25} \mathrm{~cm}^{-2}$ (SMC BEMBB). In the Milky Way, Planck Collaboration et al. (2014) found $N_{\mathrm{HI}} / \tau_{160}=1.1 \times 10^{25} \mathrm{~cm}^{-2}$ in the diffuse ISM. Our $N_{\mathrm{H}} / \tau_{160}$ values are on average a factor of $\sim 1.5$ (LMC) and $~ 4.4$ (SMC) times higher than the diffuse Milky Way ISM, which is consistent with the expectation that the gas-to-dust ratio should increase with decreasing metallicity.

Given the total NANTEN CO luminosities of $L(\mathrm{CO})_{\mathrm{LMC}}=7 \times 10^{6} \mathrm{~K} \mathrm{~km} \mathrm{~s}^{-1} \mathrm{pc}^{2}$ and $L(\mathrm{CO})_{\mathrm{SMC}}=1.7 \times$ $10^{5} \mathrm{~K} \mathrm{~km} \mathrm{~s}^{-1} \mathrm{pc}^{2}$ (using no sensitivity cuts), we find
$\alpha_{\mathrm{CO}}^{\mathrm{LMC}}=10_{-6}^{+9}$ and $\alpha_{\mathrm{CO}}^{\mathrm{SMC}}=76_{-38}^{+77}$, where all units for $\alpha_{\mathrm{CO}}$ are given in $M_{\odot}\left(\mathrm{K} \mathrm{km} \mathrm{s}^{-1} \mathrm{pc}^{2}\right)^{-1}$. Compared to the Milky Way value of $\alpha_{\mathrm{CO}}=4.3$ (Bolatto et al. 2013), the conversion factor for the LMC is $\sim 2$ times higher and the SMC is $\sim 17$ times higher. Our $\alpha_{\mathrm{CO}}$ values are comparable to the dust-based $\alpha_{\mathrm{CO}}$ found by Leroy et al. (2011) of $6.6\left(\mathrm{~K} \mathrm{~km} \mathrm{~s}^{-1} \mathrm{pc}^{2}\right)^{-1}$ and 53-85 $\left(\mathrm{K} \mathrm{km} \mathrm{s}^{-1} \mathrm{pc}^{2}\right)^{-1}$ for the LMC and SMC, respectively.

\subsubsection{Structure of the Molecular Gas}

One of the most striking results is the similarity of the structure of the molecular gas traced by dust to that traced by CO throughout the entire LMC (see Figures 2 and 4) and SMC (see Figure 3). Since our methodology only indirectly uses the CO map as a mask (see Section 3.1) the similarity is confirmation that our methodology traces the structure of the gas. Figure 4 shows that both dust modeling techniques produce maps with similar structure, although the BEMBB map tends to predict systematically lower amounts of $\mathrm{H}_{2}$.

The details of the structures traced by $\mathrm{CO}$ are different from the dust-based molecular gas map. All of the regions shown in Figure 4 show molecular gas traced by dust, but not by $\mathrm{CO}$ at the $3 \sigma$ level. This is likely a layer of self-shielded $\mathrm{H}_{2}$ where $\mathrm{CO}$ has mostly dissociated, as expected from models (Wolfire et al. 2010; Glover \& Mac Low 2011). The same is generally true for the SMC, but having only the lower resolution full coverage NANTEN ${ }^{12} \mathrm{CO}$ map $(r=2 ! 6)$ makes detailed comparison of the structure difficult. Conversely, Region 2 in Figure 4 shows a molecular gas cloud traced by $\mathrm{CO}$ and not by the dust-based method. As discussed in Leroy et al. (2009), one possible explanation is that the dust is cold and faintly emitting in the far-IR, below the sensitivity of the HERITAGE Herschel 
Region 1
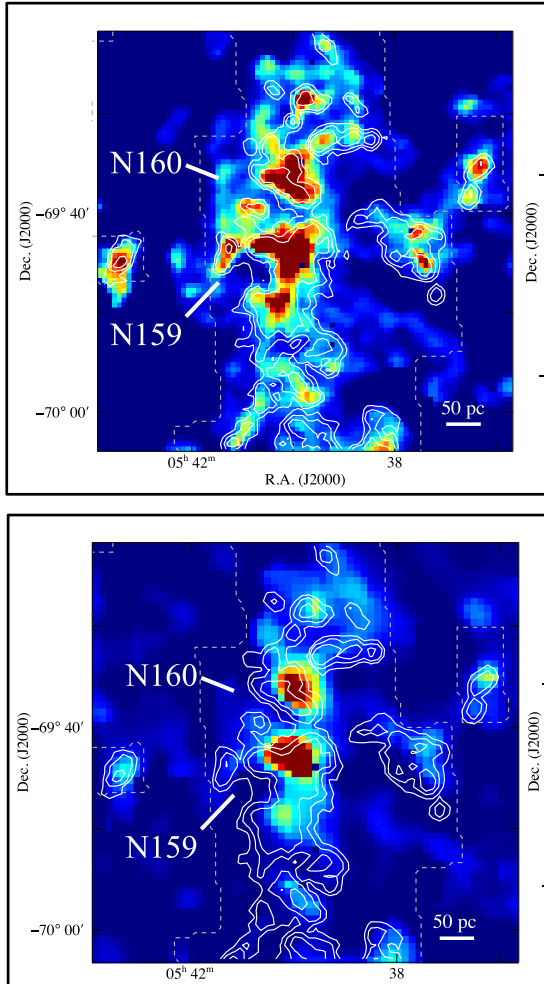

R.A. (J2000)
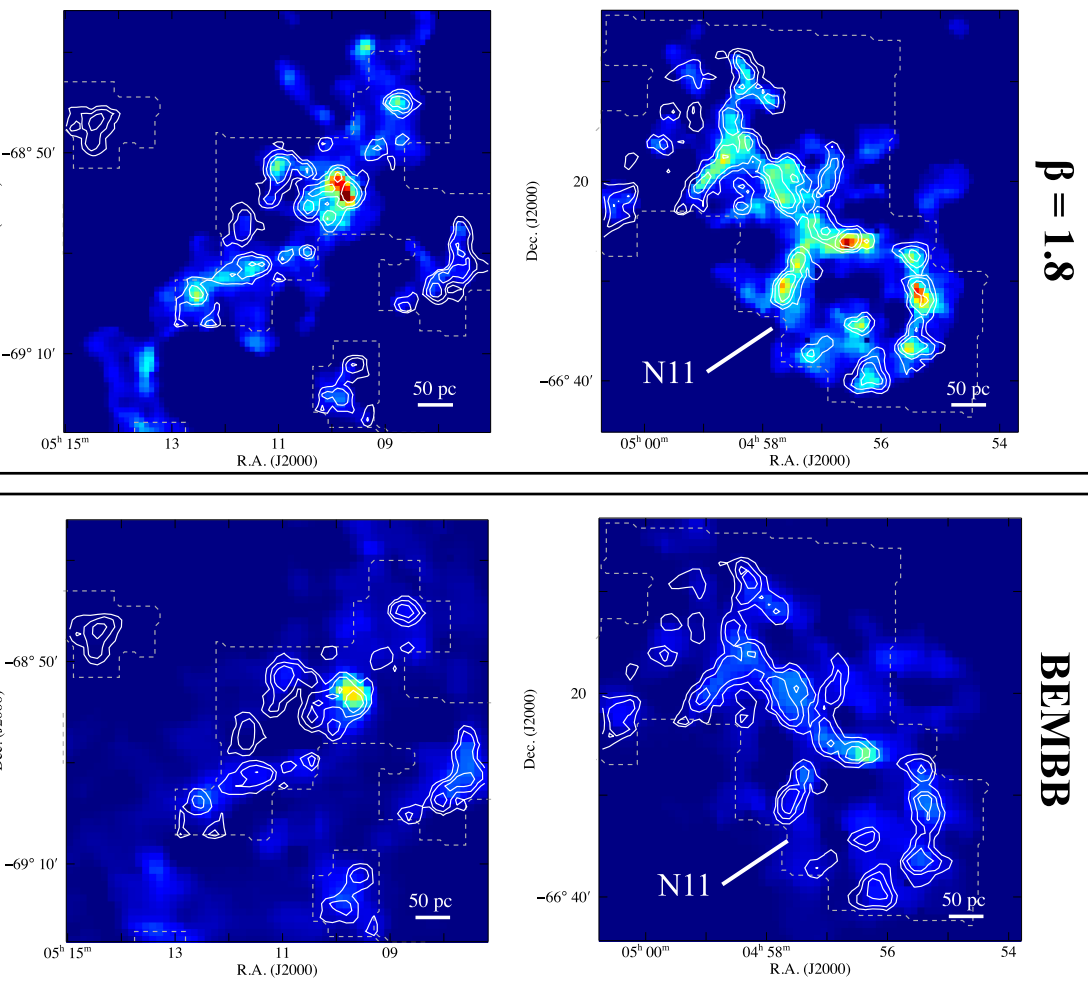

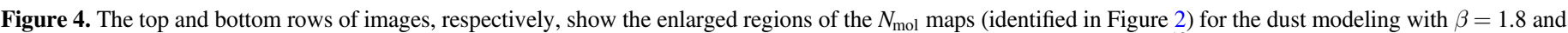

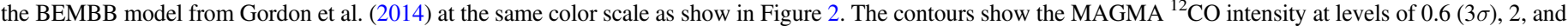

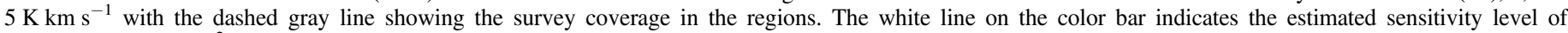

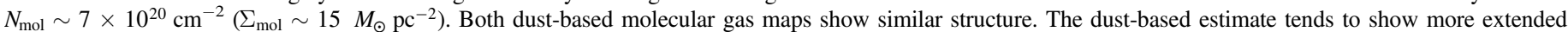

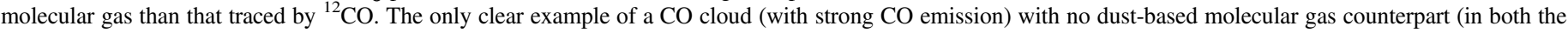
LMC and SMC) is found in the northeast of Region 2. The difference in intensity demonstrates the systematic uncertainty in the methodology.

images. The peak in the $\mathrm{CO}$ emission of this cloud is detected from $250-500 \mu \mathrm{m}$, but only weakly detected at $160 \mu \mathrm{m}$ and marginally detected $(\sim 3 \sigma)$ at $100 \mu \mathrm{m}$, consistent with the interpretation of cold dust. There are a few other detections of $\mathrm{CO}$ without a dust-based molecular gas counterpart, although the cloud in Region 2 is the clearest example with the strongest $\mathrm{CO}$ emission.

\subsubsection{Systematic Uncertainty}

The systematic uncertainty comes from the different possible assumptions that can be made in the dust modeling and the method of measuring the gas-to-dust ratio. Because the statistical errors are typically small, the systematic uncertainty dominates the total uncertainty in the molecular gas mapping methodology (Leroy et al. 2009; Bolatto et al. 2011). We present the range of our total molecular gas mass estimates $\left(M_{\mathrm{mol}}\right)$ in Table 1 (we list all $M_{\mathrm{mol}}$ estimates alongside estimates from the literature in Table 3 in Appendix D). We use the range of as a means to gauge the amount of total systematic uncertainty and we look at the variation between the two fiducial molecular gas maps (Table 1 rows 1 and 2) with different dust modeling assumptions to determine the amount of systematic uncertainty due to assumptions in the dust modeling.

The two lowest $M_{\text {mol }}$ estimates that we found use a single gas-to-dust ratio of 380 from Roman-Duval et al. (2014) and use the upper estimates for the gas-to-dust ratios for the diffuse and the dense gas from Roman-Duval et al. (2014) of $\mathrm{GDR}_{\text {diffuse }}=540$ and $\mathrm{GDR}_{\text {dense }}=330$ (see rows 6 and 10 in Table 3 in Appendix D). These maps have large regions of negative values from where the estimated total gas is less than the $\mathrm{H}_{\mathrm{I}}$, which causes only small areas of estimated $\mathrm{H}_{2}$ and the low $M_{\text {mol }}$ values, which would be due to using too low a value of the gas-to-dust ratio. The value for $M_{\text {mol }}$ with GDR $=380$ is less than the total molecular gas you would obtain by applying a Galactic CO-to- $\mathrm{H}_{2}$ conversion factor to the low resolution NANTEN CO map, which is a lower limit on the total molecular gas since a higher conversion factor should be appropriate when the $\mathrm{CO}$ structure is unresolved. We do not consider these values of $M_{\text {mol }}$ when estimating the systematic uncertainty in the total molecular gas mass.

The difference between the highest (row 5) and lowest (row 6 ) molecular gas mass is $\sim 3.5$. The minimum $M_{\text {mol }}$ estimate (row 6) comes from assuming a single gas-to-dust ratio of 540, which is the highest value found by Roman-Duval et al. (2014). This $M_{\text {mol }}$ estimate is only a factor of $\sim 1.5$ lower than using a spatially varying $\delta_{\mathrm{GDR}}$ applied to the same BEMBB dust modeling results. The maximum value comes from using the BEMBB modeling that does not remove an $\mathrm{H}_{\mathrm{I}}$ offset (row 5), which allows for a possible nonlinear relationship in $N_{\mathrm{H} \text { I }}$ versus $\tau_{160}$ (see Section 3.1.1 and Appendix B). This would be an overestimate if the relationship between $N_{\mathrm{H} \text { I }}$ versus $\tau_{160}$ is 
linear since it will artificially increase the $\delta_{\mathrm{GDR}}$ values in the maps. Allowing for a difference in the gas-to-dust ratio in the diffuse and dense gas by scaling down $\delta_{\mathrm{GDR}}$ in the dense gas reduces $M_{\text {mol }}$ by a factor of $\sim 2$ for the $\beta=1.8$ map and $\sim 1.5$ for the BEMBB map. We conclude that our molecular gas estimate is good to within a factor of $\sim 2$, which agrees with the estimates from similar methodologies by Leroy et al. (2009, 2011) and Bolatto et al. (2011).

We compare the effects of different dust modeling techniques by using $\beta=1.8$ and BEMBB maps while keeping all other aspects of the methodology the same (using a spatially varying $\left.\delta_{\mathrm{GDR}}\right)$. Figure 4 shows the difference between the molecular gas maps using the $\beta=1.8$ and BEMBB modeling (top and bottom rows, respectively). The BEMBB map is a factor of $\sim 2$ lower for molecular gas column density estimates than using the fits from the $\beta=1.8$ model. The difference in values between the two maps shows no variation as a function of $\tau_{160}$, which indicates that the dust models do not produce systematically different results in the dense gas as compared to the diffuse. The $\tau_{160}$ values from the BEMBB modeling (Gordon et al. 2014) tend to be higher than from the $\beta=1.8$ modeling, largely due to differences in the fitted dust temperatures $\left(T_{d}\right)$. The BEMBB modeling tends to fit higher $T_{d}$, which is a result of the range of $\beta$ values combined with the degeneracy between $\beta$ and $T_{d}$ (Dupac et al. 2003; Shetty et al. 2009): fitting a lower $\beta$ value to the same data will result in an increase in $T_{d}$. An increase in $\tau_{160}$ produces lower effective gas-to-dust ratio $\left(\delta_{\mathrm{GDR}}\right)$ and a lower estimate of the amount of molecular gas. Our adopted factor of $\sim 2$ systematic uncertainty is consistent with the variation seen between the two maps.

\subsubsection{Estimating the Effect of the Optical Depth of $\mathrm{H}_{\mathrm{I}}$}

We apply the statistical optical depth corrections from Stanimirović et al. (1999) for the SMC and Lee et al. (2015b) for the Milky Way to $N_{\mathrm{H} \text { I }}$ maps to estimate how accounting for optically thick $\mathrm{H}_{\mathrm{I}}$ could affect our molecular gas estimate. There is no comparable optical depth correction for the LMC, so we apply the statistical corrections for the lower metallicity SMC and higher metallicity Milky Way to estimate a range of possible effects. Applying the Lee et al. (2015b) correction to the LMC H I produces a maximum correction factor of 1.43 and shifts the top $5 \%$ of $N_{\mathrm{H}}$ from $>3.1 \times 10^{21} \mathrm{~cm}^{-2}$ to $>4.0 \times 10^{21} \mathrm{~cm}^{-2}$, whereas the Stanimirovic et al. (1999) correction produces a maximum correction factor of 1.36 and shifts the top $5 \%$ to $>3.3 \times 10^{21} \mathrm{~cm}^{-2}$. Applying the Stanimirovic et al. (1999) correction to the SMC produces a maximum correction factor of 1.48 and shifts the top 5\% of the $N_{\mathrm{H} \text { I }}$ from $>4.4 \times 10^{21} \mathrm{~cm}^{-2}$ to $>5.2 \times 10^{21} \mathrm{~cm}^{-2}$. In the LMC, both of the statistical optical depth corrections decrease the total molecular gas mass estimate by $\sim 5 \%$ while in the $\mathrm{SMC}$ it increases the total molecular gas mass by a factor of $\sim 2$, both are within our estimate of the systematic uncertainty.

The molecular gas estimate changes because the amount of $\mathrm{H}_{\mathrm{I}}$ in the diffuse regions, where we determine the effective gasto-dust ratio, increases or decreases with respect to the amount of $\mathrm{H}_{\mathrm{I}}$ in the molecular regions. Optical depth corrections in the diffuse gas will increase the effective gas-to-dust ratio and increase the estimate of the total amount of gas. If the optical depth corrections in the molecular regions are similar to the corrections in the diffuse regions, as is the case in the SMC, the total amount of gas will increase and the molecular gas estimate will increase. If the optical depth corrections in the molecular regions are larger than in the diffuse, more of the total gas estimate will be due to $\mathrm{H}$ I as opposed to $\mathrm{H}_{2}$ and the molecular gas estimate will decrease, as is the case in the LMC. Ultimately, the decrease in the molecular gas mass estimate in the LMC is negligible, which indicates that optical depth effects do not significantly contribute to our molecular gas estimate.

\subsubsection{Comparison to Previous Work}

The molecular gas maps we present are improvements upon previous dust-based $\mathrm{H}_{2}$ estimates given the availability of the Herschel data with increased sensitivity and coverage of the far-IR combined with improvements in the methodology and more extensive estimation of the systematic uncertainty. Table 1 includes the existing dust-based molecular gas mass estimates for LMC and SMC from the literature. While some of the total molecular mass values are outside the range of the estimate from this work, they can all be reconciled and explained by differences in methodology and limitations in the data. For a more detailed explanation of the differences in the $M_{\mathrm{mol}}$ estimates from previous works see Appendix D.

\subsection{Molecular Gas and Star Formation}

Understanding whether or not metallicity and galaxy mass affect the conversion of gas into stars is important for understanding galaxy evolution throughout cosmic time. The relationship between molecular gas and star formation rate has been studied extensively in nearby, high-metallicity, starforming galaxies. With the dust-based molecular gas estimates of the nearby Magellanic Clouds, we are in a unique position to probe how the relationship between the molecular gas and star formation rate behave as a function of metallicity and the size scale considered. Figure 5 shows the relationships for the LMC and SMC using the new dust-based molecular map at the highest resolution of $20 \mathrm{pc}, 200 \mathrm{pc}$ (the scale where multiple star-forming regions are being averaged), and $1 \mathrm{kpc}$ (comparable to the ${ }^{12} \mathrm{CO}$ surveys of nearby galaxies).

We compare the relationship between the molecular gas and the star formation rate in the SMC and LMC to that for the HERACLES sample of nearby galaxies by Leroy et al. (2013b). The HERACLES sample resolves the galaxies and compares the gas and star formation at a resolution of $\sim 1 \mathrm{kpc}$. Figure 6 shows that the LMC and SMC data (convolved to a comparable resolution of $1 \mathrm{kpc}$ ) lie within the scatter in the data for high-metallicity, star-forming galaxies, although above the main cluster of data points for a given molecular gas surface density.

\subsubsection{Molecular Gas Depletion Time}

A convenient way to quantify the relationship between molecular gas and star formation is in terms of the amount of time it would take to deplete the current reservoir of gas given the current rate of star formation, the molecular gas depletion time:

$$
\tau_{\text {dep }}^{\text {mol }}=\Sigma_{\text {mol }} / \Sigma_{\mathrm{SFR}}
$$

The data for the LMC and SMC appear consistent with a welldefined depletion time. We find average molecular gas depletion times at $1 \mathrm{kpc}$ scales of $\sim 0.4 \mathrm{Gyr}$ in the LMC and $\sim 0.6$ Gyr in the SMC. Weighting the average of $\tau_{\text {dep }}^{\text {mol by the }}$ 

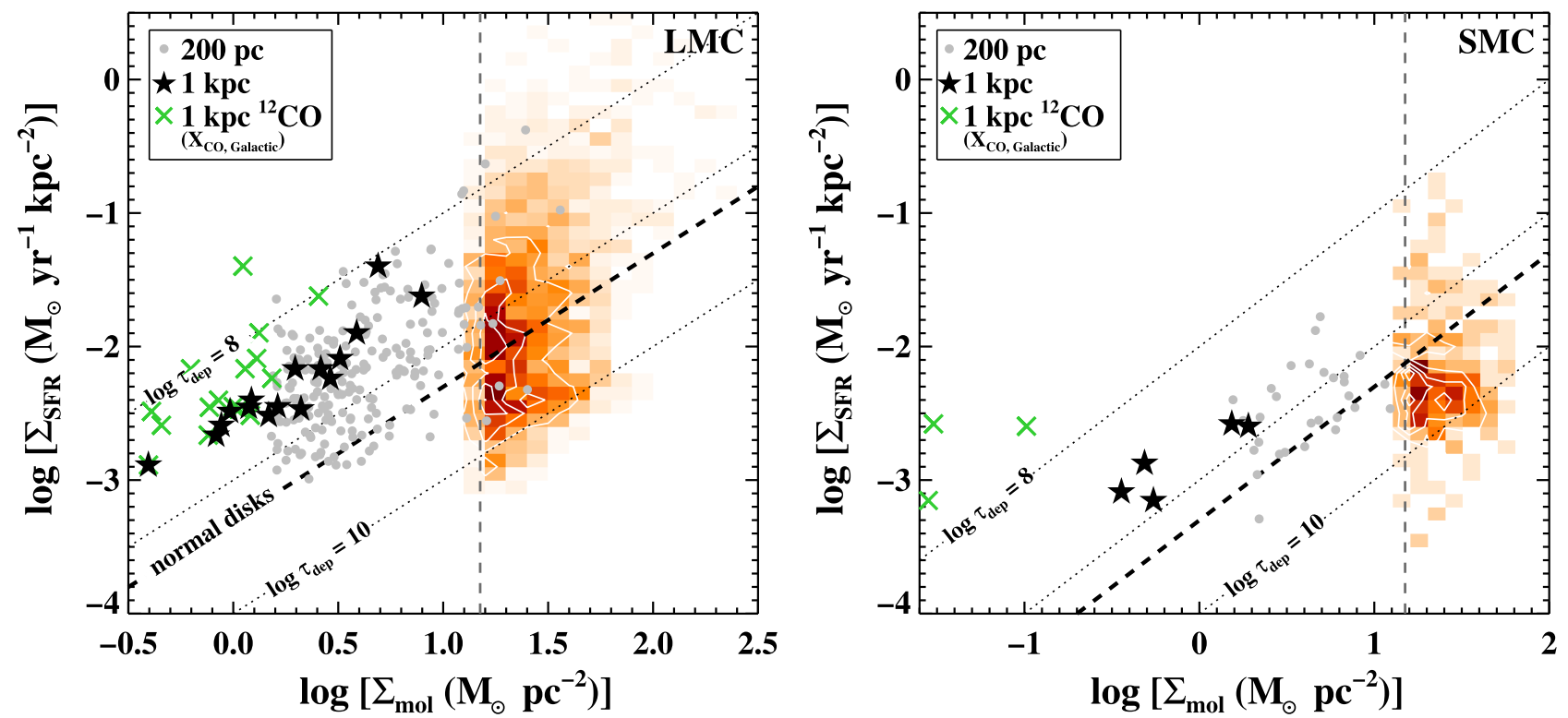

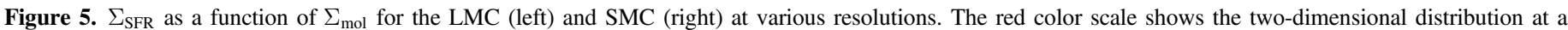

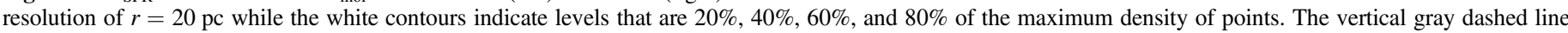

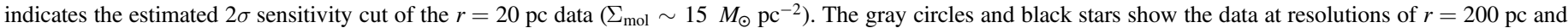

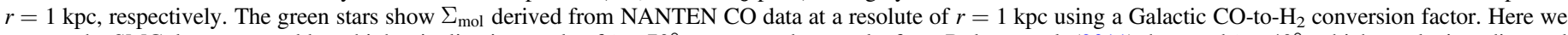

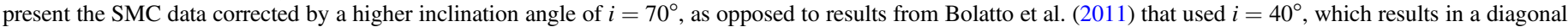

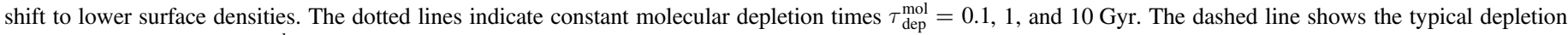
time for normal galaxies $\tau_{\text {dep }}^{\text {mol }} \sim 2$ Gyr (Bigiel et al. 2008, 2011; Rahman et al. 2012; Leroy et al. 2013b).

molecular gas mass and star formation rate does not significantly affect the averages at $1 \mathrm{kpc}$ scales; at $200 \mathrm{pc}$ scales, weighting of the average typically changes the value of

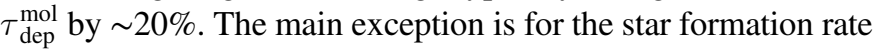
weighted $\tau_{\text {dep }}^{\text {mol }}$ average in the LMC, which is shorter by $\sim 50 \%$ and likely due to the significant contribution of 30 Doradus at these scales. The range of possible molecular gas depletion times given the factor of up to $\sim 2$ systematic uncertainty in the molecular gas estimate is $\sim 0.2-1.2$ Gyr. This is shorter than the molecular gas depletion time found for the SMC by Bolatto et al. (2011) of $\tau_{\text {dep }}^{\text {mol }} \sim 1.6 \mathrm{Gyr}$ at $1 \mathrm{kpc}$ resolution, but within the factor of 2 systematic uncertainty on both estimates. The molecular gas depletion time found in the Magellanic Clouds is lower than the average value of $\sim 2$ Gyr for nearby normal disk galaxies at comparable $\sim 1 \mathrm{kpc}$ size scales, but within the range of observed values for the STING sample (Rahman et al. 2012) and the larger HERACLES sample (Bigiel et al. 2008, 2011; Leroy et al. 2013b).

Figure 7 shows that the median $\tau_{\text {dep }}^{\text {mol }}$ is $\sim 2-3$ Gyr at the highest resolution of $20 \mathrm{pc}$. The molecular gas depletion time changes with resolution because the peaks in the molecular gas are physically separated from the peaks in the star formation rate at scales where the star-forming regions are spatially resolved. The tendency of low star formation rates at the peaks in the molecular gas, and low to no molecular gas at the peaks in the star formation rate ( $\tau_{\text {dep }}^{\mathrm{mol}}$ is only defined for regions with

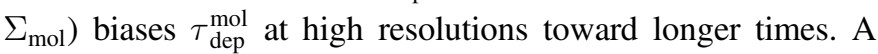
scale of $200 \mathrm{pc}$ is typically large enough to include both the recent star formation and the molecular gas and sample starforming regions at a range of evolution stages (Schruba et al. 2011). While the molecular gas depletion time gets closer to the integrated $\tau_{\text {dep }}^{\text {mol }}$ value at a scale of $200 \mathrm{pc}$, the median $\tau_{\text {dep }}^{\text {mol }}$ reaches the integrated value at $\sim 500 \mathrm{pc}$ in the LMC and SMC.

The lower metallicities of the SMC and LMC and the lack of a metallicity bias in our dust-based molecular gas estimate allow us to investigate whether there is any trend in $\tau_{\text {dep }}^{\text {mol with }}$ metallicity. Figure 9 shows that there is no clear trend in the average molecular gas depletion times when comparing the LMC and SMC to the HERACLES sample of galaxies. Leroy et al. (2013b) also saw no trend with metallicity as long as they allowed for a variable $\mathrm{CO}-$ to- $\mathrm{H}_{2}$ conversion factor. We also compare our measurements to the integrated $\tau_{\text {dep }}^{\text {mol }}$ using a metallicity-dependent CO-to- $\mathrm{H}_{2}$ conversion factor for the Herschel Dwarf Galaxy Survey (DGS; Cormier et al. 2014). Over the range of metallicities studied, the main cause of variations in the molecular gas depletion time does not appear to be metallicity.

\subsection{Correlation Between Gas and Star Formation Rate from 20 pc to $1 \mathrm{kpc}$ Size Scales}

We use the Spearman's rank correlation coefficient to quantitatively gauge how well the gas correlates with star formation rate at different size scales. Spearman's rank correlation coefficient $\left(r_{s}\right)$ measures the degree to which two quantities monotonically increase $\left(r_{s}>0\right)$ or decrease $\left(r_{s}<0\right)$. We computed the $3 \sigma$ confidence intervals using the Fisher $z$ transformation, which is appropriate for bivariate normal distributions. Figure 8 shows the rank correlation coefficient as a function of resolution for the relationship between star formation rate and molecular gas and atomic gas.

The change in the rank correlation coefficient with resolution is similar for both the LMC and SMC. As expected for atomicdominated galaxies, the correlation of $\Sigma_{\text {gas }}$ versus $\Sigma_{\text {SFR }}$ follows that of $\Sigma_{\mathrm{HI}}$ and $\Sigma_{\mathrm{SFR}}$, therefore we only show $\Sigma_{\mathrm{HI}}$ versus $\Sigma_{\mathrm{SFR}}$ in Figure 8 . The correlation between $\Sigma_{\mathrm{H} \text { I }}$ versus $\Sigma_{\mathrm{SFR}}$ in both 


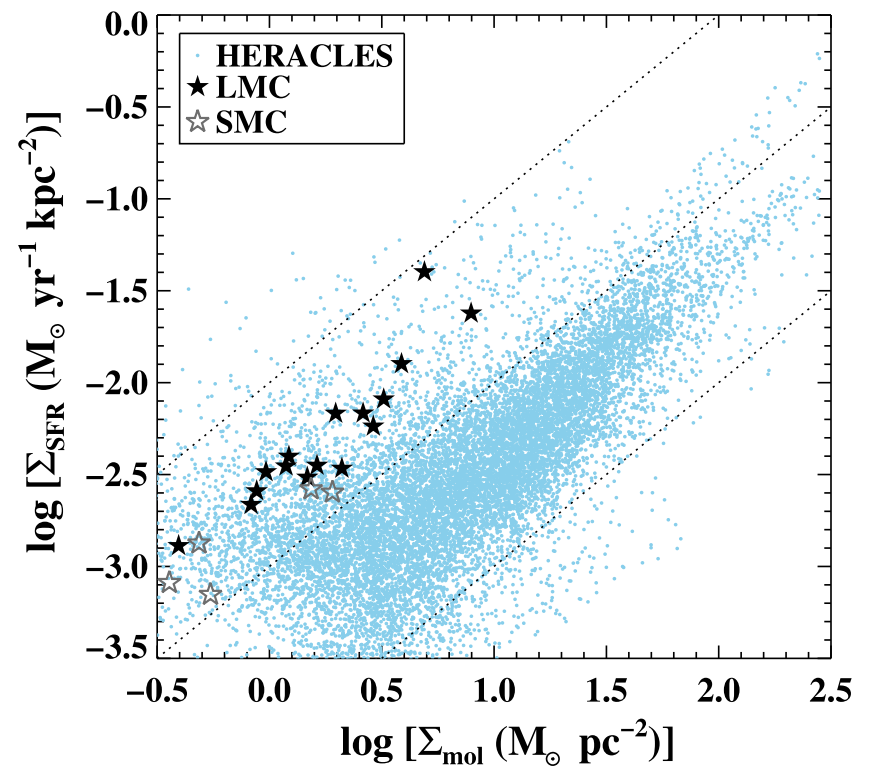

Figure 6. $\Sigma_{\mathrm{SFR}}$ vs. $\Sigma_{\mathrm{mol}}$ for the $r \sim 1 \mathrm{kpc}$ data from the HERACLES sample of nearby star-forming galaxies (Leroy et al. 2013b) (blue), where the $\Sigma_{\mathrm{mol}}$ is estimated using ${ }^{12} \mathrm{CO}$ with a Galactic $\mathrm{CO}-$ to- $\mathrm{H}_{2}$ conversion factor. The $r \sim$ $1 \mathrm{kpc}$ data for the LMC (filled stars) and SMC (open stars) are over plotted. The LMC and SMC points fall within the full distribution for the HERACLES sample, but offset above the main distribution.

the LMC and SMC is high $\left(r_{s} \sim 0.6-0.7\right)$ at the smallest size scale of $20 \mathrm{pc}$ and remains high across all size scales. The correlation of $\mathrm{H}_{\mathrm{I}}$ with the star formation rate, even at small spatial scales, is due to the extended nature of both components combined with the general trend that regions with more total gas have more star formation and more molecular gas.

The $\Sigma_{\mathrm{H}_{2}}$ versus $\Sigma_{\mathrm{SFR}}$ distribution reaches the maximum correlation coefficient of $r_{s} \sim 0.9$ at a size scales $\sim 200 \mathrm{pc}$, past which it is better correlated than the relationship with $\mathrm{HI}$. While the $\mathrm{HI}_{\mathrm{I}}$ is correlated with the star formation rate tracer, we see that molecular gas is best correlated with recent star formation in the LMC and SMC at size scales $\gtrsim 200 \mathrm{pc}$. The $200 \mathrm{pc}$ scale indicates the average size scale where both molecular gas and the star formation rate tracer, $\mathrm{H} \alpha$, are found together and enough independent star-forming regions at different evolutionary stages (i.e., different ratios of $\mathrm{H} \alpha$ to molecular gas) are averaged together. While the correlation peaks at $200 \mathrm{pc}$, the average molecular gas depletion time decreases until it reaches the integrated value at a size scale of $\sim 500-700 \mathrm{pc}$ in the LMC and SMC. The molecular gas and star formation rate tracer have a strong positive correlation, stronger than that with $\mathrm{H}$, supporting the physical connection between molecular gas and recent massive star formation.

\section{DISCUSSION}

We discuss our findings on the relationship between gas and star formation in the Magellanic Clouds using our new dustbased molecular gas maps. By comparing our results to existing observational studies of mainly massive, high-metallicity, molecular-dominated galaxies, simulations, and theoretical models of star formation, we provide insight into the physical mechanisms that drive star formation.

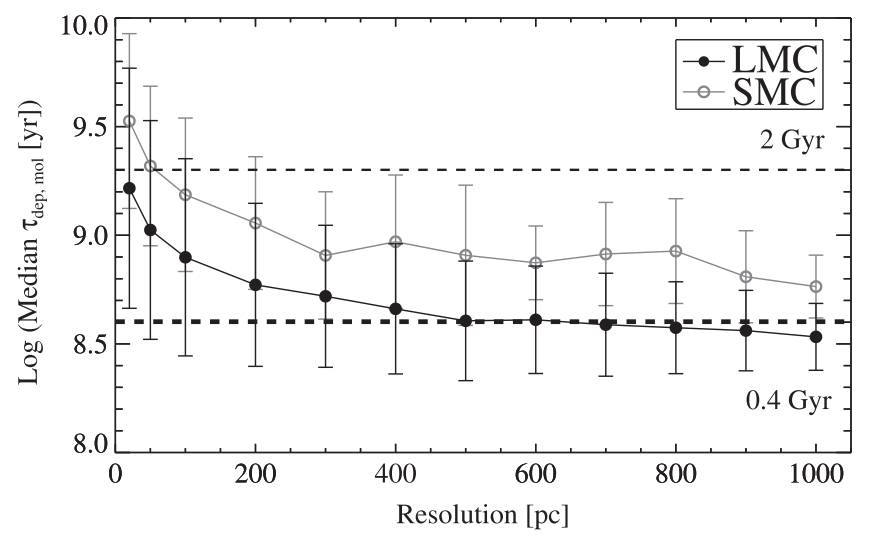

Figure 7. Median molecular gas depletion time as a function of resolution. Black filled and open gray circles show the data for the LMC and SMC, respectively. The error bars show $1 \sigma$ on the mean. The upper dashed line shows $\tau_{\text {dep }}^{\text {mol }}=2 \mathrm{Gyr}$, the average for normal galaxies, and the lower dashed line shows $\tau_{\text {dep }}^{\text {mol }}=0.4 \mathrm{Gyr}$, the integrated depletion time for both the LMC and SMC. The LMC and SMC $\tau_{\text {dep }}^{\text {mol }}$ reach the integrated value of $\sim 0.4$ Gyr and $\sim 0.6 \mathrm{Gyr}$, respectively, at large $(>500 \mathrm{pc})$ scales.

\section{1. $\tau_{\mathrm{dep}}^{\mathrm{mol}}$ in the Magellanic Clouds}

The range of possible molecular gas depletion times for the LMC and SMC at $1 \mathrm{kpc}$ scales given the systematic uncertainty in our estimate of the molecular gas of $\sim 0.2-1.2 \mathrm{Gyr}$ falls below the average $\sim 2 \mathrm{Gyr}$ found for nearby normal disk galaxies. This is consistent with the previous work by Bolatto et al. (2011) that found $\tau_{\text {dep }}^{\mathrm{mol}}=1.6 \mathrm{Gyr}$ at $1 \mathrm{kpc}$ scales in the SMC using similar dust-based molecular gas estimates, with the value being higher due to a higher estimate of the molecular gas. The shorter molecular gas depletion times do not appear to be directly due the lower metallicities as there is no trend in

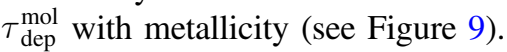

The other remaining environmental factors, besides metallicity, that could affect the ratio of the amount of molecular gas to the amount of current star formation are the lower galaxy masses of the Magellanic Clouds and the interaction between the LMC, SMC, and Milky Way (Besla et al. 2012). Lower mass galaxies tend to have lower dark matter and stellar densities, making them more susceptible to stochastic bursts of star formation. Both the star formation histories of the SMC and LMC (Harris \& Zaritsky 2004, 2009) indicate that there have been recent bursts in star formation in both galaxies. A burst in star formation over a short period of time could lead to a depletion of the molecular gas reservoir combined with higher star formation rates that together can produce low $\tau_{\text {dep }}^{\text {mol }}$ values.

The molecular gas depletion time in M33 is $~ 0.5$ Gyr when the diffuse $\mathrm{H} \alpha$ emission is included (Schruba et al. 2010), which is comparable to our measurements of the Magellanic Clouds. If the diffuse ionized gas (DIG) is removed from the $\mathrm{H} \alpha$ emission, then the molecular gas depletion time increases to $\sim 1$ Gyr. This highlights the importance of understanding the connection between the DIG and recent massive star formation as it represents a significant fraction of the $\mathrm{H} \alpha$ emission and changes $\tau_{\text {dep }}^{\text {mol }}$ Rahman et al. (2011) found a similar increase in

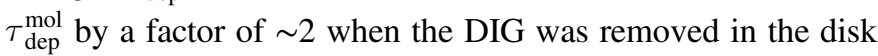
galaxy NGC 4254. If the diffuse ionized component is excluded in the star formation rate determination, the $\tau_{\text {dep }}^{\text {mol in }}$ M33, LMC, and SMC is $\sim 1$ Gyr. 


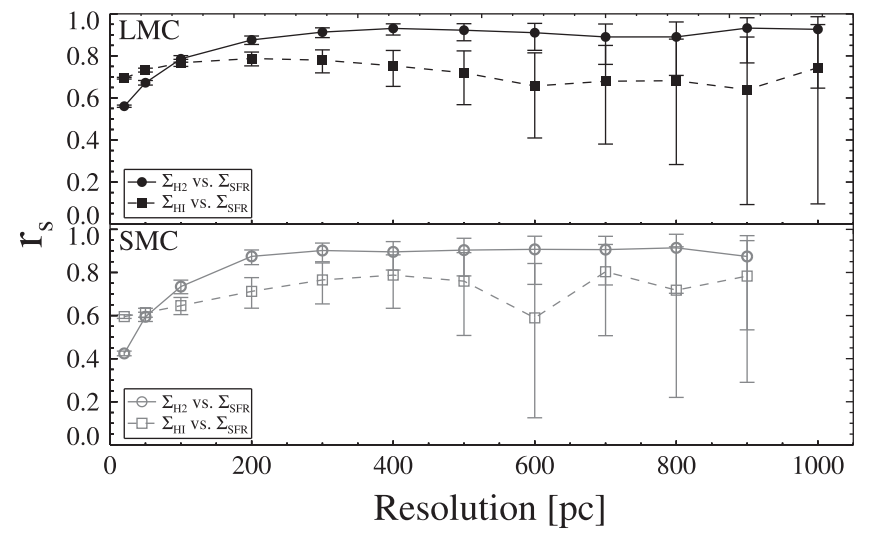

Figure 8. The Spearman rank correlation coefficient $\left(r_{s}\right)$ as a function of image resolution for the $\Sigma_{\mathrm{H}_{2}}$ vs. $\Sigma_{\mathrm{SFR}}$ (circles with solid line) and $\Sigma_{\mathrm{HI}}$ vs. $\Sigma_{\mathrm{SFR}}$ (squares with dashed line) distributions. The top plot shows the rank correlations for the LMC and the bottom show those for the SMC. The error bars show the $99.75 \%$ confidence interval $(\sim 3 \sigma)$ of the measured rank correlation coefficient. The correlation between $\mathrm{H}_{\mathrm{I}}$ and the star formation rate remains at a constant, high level of $r_{s} \sim 0.7$ across size scales in part due to the extended nature of both the $\mathrm{H}$ i gas and $\mathrm{H} \alpha$ emission that dominates the star formation rate. The correlation between $\mathrm{H}_{2}$ and star formation rate reaches a maximum value of $r_{s} \sim 0.9$ at a size scale of $200 \mathrm{pc}$, which is the expected size scale to average over enough individual star-forming regions to sample a range of evolutionary states.

Like the Magellanic Clouds, M33 is low-mass, atomicdominated, and has likely interacted with M31 within the past 0.5-2 Gyr (Davidge \& Puzia 2011). The LMC, SMC, and M33 show evidence for bursts in the star formation history within the last Gyr and the most recent epochs show lower star formation rates, which suggest that the star-forming gas reservoir has been depleted. The observed shorter depletion times appear to be caused by catching these galaxies after a period of higher star formation rate and does not necessarily indicate that these low-mass, low-metallicity galaxies are forming stars differently from normal disk galaxies.

Saintonge et al. (2011) also found that for the volumelimited COLD GASS survey, lower stellar mass galaxies $\left(\sim 10^{10} M_{\odot}\right)$ had shorter depletion times of $\sim 0.5 \mathrm{Gyr}$. While consistent with the integrated depletion times in the LMC and $\mathrm{SMC}$, the data are not completely comparable since a value for the CO-to- $\mathrm{H}_{2}$ conversion factor has to be assumed and singledish CO observations from the COLD GASS survey will mainly detect the central regions of the galaxies. Saintonge et al. (2011) conjecture that the shorter depletion time is due to the tendency for smaller galaxies to have more "bursty" star formation. Similarly, Cormier et al. (2014) suggest that the observed short molecular gas depletion depletion times for their DGS sample of dwarf galaxies are due to recent bursts in star formation. Kauffmann et al. (2003) found that low redshift galaxies with stellar mass $<3 \times 10^{10} M_{\odot}$ in the Sloan Digital Sky Survey have younger stellar populations and that the star formation histories are correlated with the stellar surface density, also indicative of recent bursts in star formation as seen in the LMC, SMC, and M33. In Figure 10, we show the

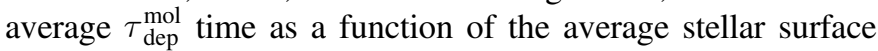
density $\left(\Sigma_{*}\right)$ for the LMC, SMC, and the HERACLES sample of galaxies and see that all of the low molecular depletion times are found at low $\Sigma_{*}$. The fact that low-mass galaxies are more susceptible to stochastic star formation can produce bursts in

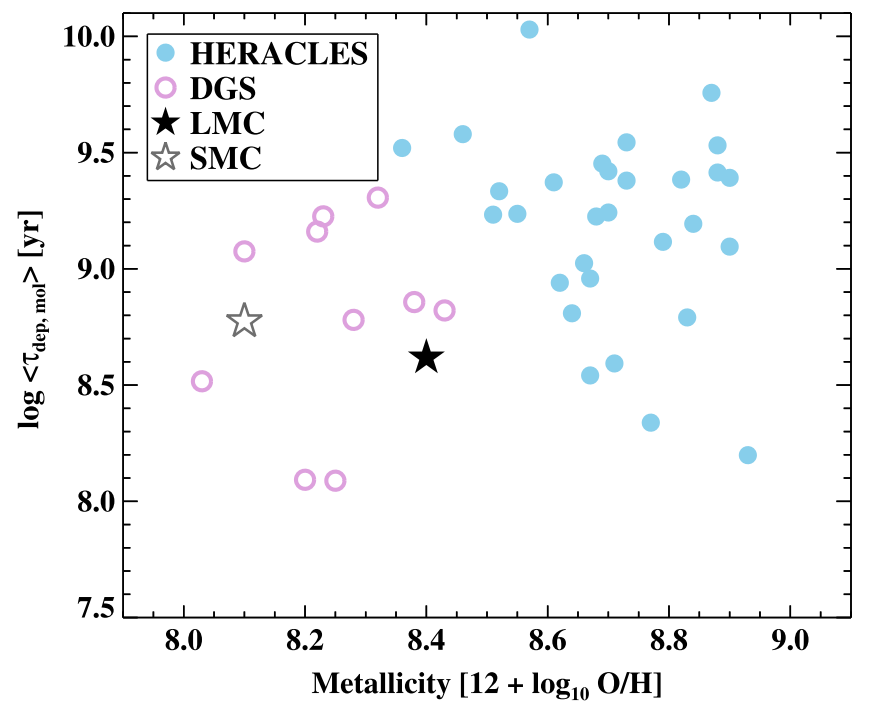

Figure 9. The galaxy-averaged molecular gas depletion time $\left(\left\langle\Sigma_{\mathrm{mol}}\right\rangle /\left\langle\Sigma_{\mathrm{SFR}}\right\rangle\right)$ with metallicity for the HERACLES sample (light blue points), LMC (black filled stars), and SMC (gray open stars). We have taken the average $\Sigma_{\text {mol }}$ and $\Sigma_{\text {SFR }}$ of the $1 \mathrm{kpc}$ LMC and SMC data, which are comparable measurements to the $\sim 1 \mathrm{kpc}$ resolution HERACLES data. We also include the integrated molecular gas depletion times $\left(M\left(\mathrm{H}_{2}\right) /\right.$ star formation rate) from the DGS using a metallicity-dependent CO-to- $\mathrm{H}_{2}$ conversion factor from Cormier et al. (2014). While there is a large amount of scatter, there does not appear to be any strong trend with metallicity.

star formation (Hopkins et al. 2014) and lead to shorter molecular gas depletion times.

\subsection{Physical Interpretation of the Scatter in $\tau_{\mathrm{dep}}^{\mathrm{mol}}$}

The scatter in the $\Sigma_{\text {mol }}-\Sigma_{\text {SFR }}$ relationship, which we quantify in terms of the scatter in $\log \tau_{\text {dep }}^{\text {mol }}$, can be produced by both physical mechanisms and the imperfect nature of the observable tracers of the physical quantities. The previous observational work that focused on the scatter in $\tau_{\text {dep }}^{\text {mol }}$, or the "break down" of the $\Sigma_{\text {mol }}-\Sigma_{\text {SFR }}$ relationship, by Schruba et al. (2010), Verley et al. (2010), and Onodera et al. (2010), studied the $\Sigma_{\text {mol }}-\Sigma_{\text {SFR }}$ relationship in M33 over $\gtrsim 100$ pc size scales. Schruba et al. (2010) compared $\tau_{\text {dep }}^{\text {mol }}$ found for apertures centered on $\mathrm{CO}$ peaks to apertures centered on $\mathrm{H} \alpha$ peaks for various aperture sizes from 75 to $1200 \mathrm{pc}$ and found that the $\tau_{\text {dep }}^{\text {mol }}$ values differed for $\mathrm{CO}$ and $\mathrm{H} \alpha$ peaks for $\lesssim 300 \mathrm{pc}$ size scales. There are a number of possible causes of the difference between the $\mathrm{CO}$ and $\mathrm{H} \alpha$ molecular gas depletions times: a difference in evolutionary stage of the star-forming region, drift of the young stars from their parent cloud, actual variation in $\tau_{\text {dep }}^{\text {mol }}$ differences in how the observables map to physics quantities, and noise in the maps. Schruba et al. (2010) identify the evolution of individual star-forming regions as the likely cause for the variations.

At high resolution (scales of $\sim 20-50 \mathrm{pc}$ ), the star formation and molecular gas are resolved into discrete regions that span a range evolutionary stages (e.g., Kawamura et al. 2009; Fukui \& Kawamura 2010) and have different ratios of molecular gas to star formation rate tracers. Averaging over larger size scales samples regions at a range of evolutionary stages resulting in a "time-averaged" $\tau_{\text {dep }}^{\text {mol }}$. The change in the scatter in the molecular gas depletion time $(\sigma)$ with resolution informs us about whether the star-forming regions are spatially correlated due to synchronization of star formation by a large-scale 
process. We see this effect in Figure 5; the scatter in $\tau_{\text {dep }}^{\text {mol }}$ decreases as the size scales are increased.

Theoretical studies can be used to explore which mechanism produces the scatter in the $\Sigma_{\text {mol }}-\Sigma_{\text {SFR }}$ relationship. We compare our results in the SMC and LMC to the hydrodynamical simulations of galaxies by Feldmann et al. (2011) and to the analytical model by Kruijssen \& Longmore (2014). Both provide predictions of the amount of scatter in the $\Sigma_{\text {mol }}-\Sigma_{\text {SFR }}$ relationship for small size scales $(50 \mathrm{pc}$ for predictions from Kruijssen \& Longmore 2014, and 300 pc for the simulations from Feldmann et al. 2011) and how the scatter changes with size scale.

The simulations by Feldmann et al. (2011) show that the time-averaging of the star formation rate (or, our inability to measure the instantaneous star formation rate) combined with $\Sigma_{\text {mol }}$ estimates that are instantaneous alone can generate most of the scatter observed in the $\Sigma_{\mathrm{SFR}}-\Sigma_{\mathrm{H}_{2}}$ relation. If we possessed a perfect, instantaneous tracer of the star formation rate, then we would expect to see high star formation rates while there is still a large amount of molecular gas. As the molecular gas is depleted and destroyed by the previous episode of star formation, both the molecular gas and star formation rate would decrease. Instead, we observe the tracers of the star formation rate (namely $\mathrm{H} \alpha$ ) peak when the molecular gas is partially or mostly dissipated because the tracers show the average star formation rate over up to $\sim 10 \mathrm{Myr}$ (Kennicutt \& Evans 2012). The time evolution of star-forming regions alone does not cause the observed offset between the observed star formation rate and molecular gas, rather the time-averaging of the star formation rate combined with the time evolution of star-forming regions produces different ratios of molecular gas to star formation rate and scatter in $\tau_{\text {dep }}^{\mathrm{mol}}$. Hony et al. (2015) show evidence of this effect in the N66 region of the SMC where the star formation rate from $\mathrm{H} \alpha$ disagrees with that from pre-main sequence stars at small $(\sim 6-150 \mathrm{pc})$ size scales.

Kruijssen \& Longmore (2014) quantify how the scatter in the $\Sigma_{\text {gas }}-\Sigma_{\text {SFR }}$ relationship should change with the size scale due to the incomplete statistical sampling of independent starforming regions, including the effect of the different timescales associated with the gas and star formation tracers discussed by Feldmann et al. (2011), and add the additional scatter associated with incomplete sampling of star formation rate tracers from the initial stellar mass function (IMF), and the spatial drift between gas and stars. The model requires having an estimate of the lifetime of GMCs $\left(t_{\text {gas }}\right)$, the timescale for the star formation rate tracer $\left(t_{\text {stars }}\right)$, the time where both the gas and star formation rate overlap $\left(t_{\text {over }}\right)$, the typical separation between independent star-forming regions $(\lambda)$, the flux ratio between peaks in the overlap phase and in isolation for the gas and star formation $\left(\beta_{1}, \beta_{2}\right)$, the scatter due to the time evolution of gas and star formation flux $\left(\sigma_{\text {evol,1g }}, \sigma_{\text {evol,1s }}\right)$, the scatter due to the mass spectrum $\left(\sigma_{\mathrm{MF}}\right)$, and the observational error $\left(\sigma_{\mathrm{obs}}\right)$. The predictions for the scale dependence of the scatter in the gas depletion time from Kruijssen \& Longmore (2014) agree with the predictions by Feldmann et al. (2011) at size scales $>300$ pc where the two are directly comparable. Kruijssen \& Longmore (2014) find that the scatter varies from $\sim 0.9$ dex at $50 \mathrm{pc}$ scales to $\sim 0.2 \mathrm{dex}$ at $1 \mathrm{kpc}$. The trend in the prediction of scatter (valid for their fiducial parameter values for disks and dwarfs) is consistent with the observation from the

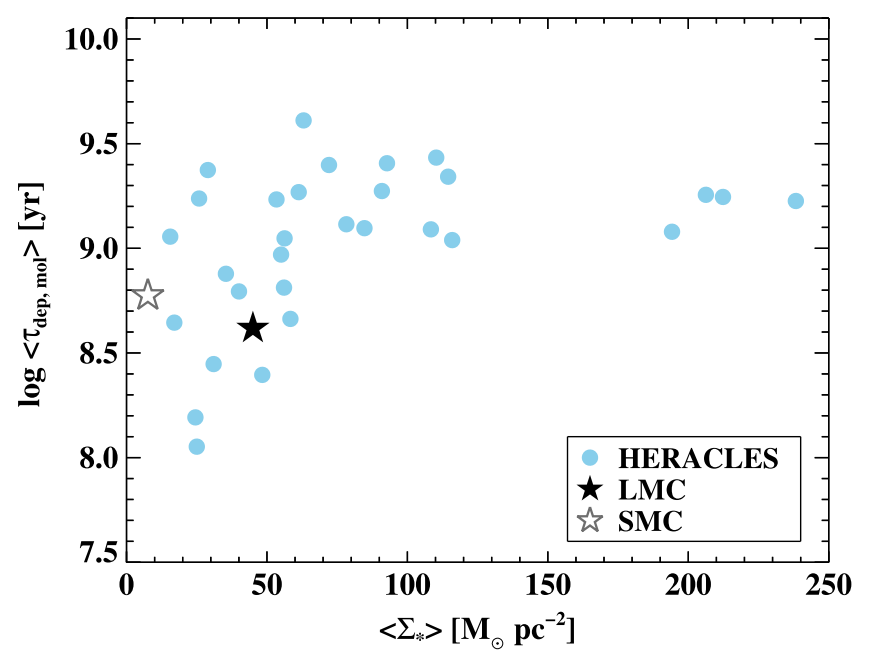

Figure 10. Average molecular gas depletion time as a function of the average stellar surface density for the disk-averaged HERACLES sample (Leroy et al. $2013 b$ ) in blue with the filled and open stars showing the galaxy-averaged data for the LMC and SMC, respectively. The HERACLES sample data used a Galactic CO-to- $\mathrm{H}_{2}$ conversion factor and $\Sigma_{*}$ was measured for the HERACLES sample, LMC, and SMC using $I_{3.6 \mu \mathrm{m}}$ from Spitzer and the conversion from Zibetti et al. (2009). We see that the LMC and SMC points

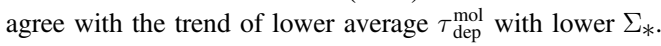

HERACLES galaxies (Leroy et al. 2013b) and M33 (Schruba et al. 2010).

For the LMC and SMC predictions of the scatter from Kruijssen \& Longmore (2014), we use estimates of the input parameters based on observational data when possible. For both the LMC and SMC, we set $t_{\text {stars }}=6 \mathrm{Myr}$ based on the lifetime of $\mathrm{H} \alpha$, make the assumption that $\beta_{1}, \beta_{2}=1$, and set $\lambda=150 \mathrm{pc}$, the typical Toomre length (for $\Sigma_{\text {gas }} \sim 10 M_{\odot} \mathrm{pc}^{-2}$ and $\Omega \sim 0.03 \mathrm{Myr}^{-1}$ ). In the LMC, we use the results of Kawamura et al. (2009) to set $t_{\mathrm{gas}}=26 \mathrm{Myr}$ and $\sigma_{\mathrm{MF}}=0.4 \mathrm{dex}$ (the mean logarithmic scatter of the Class I GMC mass). For the upper limit in the LMC, we take $t_{\text {over }}=0 \mathrm{Myr}$ and $\sigma_{\text {evol,1g }}$, $\sigma_{\text {evol,1s }}=0.3 \mathrm{dex}$, based on a linear time evolution to or from zero. For the lower limit in the LMC, we adopt $t_{\text {over }}=3 \mathrm{Myr}$ (the supernova timescale) and $\sigma_{\text {evol,1g }}, \sigma_{\text {evol,1s }}=0.15 \mathrm{dex}$; while it can vary from $0-0.3$ dex, half the amount of scatter as linear evolution is a reasonable lower limit since the parameters must be $>0$ due to the existence of molecular clouds without massive stars and $\mathrm{H}_{\text {II }}$ regions without molecular clouds. We note that Kruijssen \& Longmore (2014) assume that the galactic star formation rate is roughly constant over the entire lifetime of the GMCs ( 30 Myr), which stands in contrast to the multiple bursts over the past $\sim 50 \mathrm{Myr}$ identified in the star formation history of the both LMC (Harris \& Zaritsky 2009) and SMC (Harris \& Zaritsky 2004).

In the LMC, where the morphology is more clearly a disk and the metallicity is not much lower than solar metallicity, we observe scatter at the level of $\sim 0.45 \mathrm{dex}$ at $\sim 100 \mathrm{pc}$ and $\sim 0.18$ dex at $\sim \mathrm{kpc}$ scales. The Feldmann et al. (2011) simulations show that the behavior of the scatter in $\log \tau_{\text {dep }}^{\text {mol }}$ with an averaging size scale from $\sim 100-1000 \mathrm{pc}$ for the solar metallicity and radiation field are remarkably similar to the observations for the LMC. The simulations from Feldmann et al. (2011) predict scatter of $\sim 0.4-0.6 \mathrm{dex}$ at $\sim 100 \mathrm{pc}$ scales and $\sim 0.1-0.3 \mathrm{dex}$ at $\sim \mathrm{kpc}$ scales for their fiducial solar metallicity simulations (across the range of their parameter exploration). The Kruijssen \& Longmore (2014) model 
produces a range in the predicted scatter in $\log \tau_{\text {dep }}^{\text {mol }}$ in the LMC of $0.46-0.51 \mathrm{dex}$ at $100 \mathrm{pc}$ scales and $0.19-0.23 \mathrm{dex}$ at $1 \mathrm{kpc}$ scales, which are comparable to the results from the Feldmann et al. (2011) and close to the observed values for the LMC (see Figure 11). The dominant source of scatter at large $(>100 \mathrm{pc})$ size scales for the lower limit predictions (closest to the observations) from the Kruijssen \& Longmore (2014) model comes from the Poisson statistics of the number of times each evolutionary phase of star formation is sampled, which is determined primarily by the timescale of the star formation rate tracer, the lifetime of GMCs, and the separation between starforming regions. The similarity between our observations and both the Feldmann et al. (2011) simulation and Kruijssen \& Longmore (2014) model at large $(>100 \mathrm{pc})$ size scales, where both are comparable and individual star-forming regions are unresolved, supports the interpretation that the scatter in the $\Sigma_{\text {mol }}-\Sigma_{\text {SFR }}$ relationship can be largely attributed to star formation rate tracers that time-average the "true" or instantaneous star formation rate.

\subsubsection{Scatter in $\tau_{\mathrm{dep}}^{\mathrm{mol}}$ as a Function of Size Scale}

As a means to quantify the behavior of scatter with different size scales, Feldmann et al. (2011) fit a power law to the relationship between size scale and the scatter in $\log \tau_{\text {dep }}^{\text {mol }}$. Leroy et al. (2013b) used a subset of nearby HERACLES

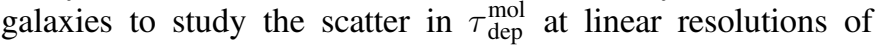
$0.6-2.4 \mathrm{kpc}$, which we can compare to our results in the Magellanic Clouds spanning linear resolutions of $0.02-1 \mathrm{kpc}$. Following Feldmann et al. (2011) and Leroy et al. (2013b), we quantify the scale dependence of the scatter in $\tau_{\text {dep }}^{\text {mol }}$ in the LMC and SMC by

$$
\sigma(l)=\sigma_{100}\left(\frac{l}{100 \mathrm{pc}}\right)^{-\gamma}
$$

where $l$ is the spatial resolution, $\sigma_{100}$ is the scatter in $\log \left(\tau_{\text {dep }}^{\mathrm{mol}}\right)$ at $100 \mathrm{pc}$ resolution, and the power-law index $\gamma$ measures the rate that the scatter changes with resolution $(\gamma=1$ for uncorrelated star formation in a disk). ${ }^{15} \mathrm{We}$ fit only resolutions greater than $100 \mathrm{pc}$, since below that resolution $\log \left(\tau_{\mathrm{dep}}^{\mathrm{mol}}\right)$ will be biased by negative and zero values. Figure 11 shows how the scatter in $\tau_{\text {dep }}^{\text {mol }}$ changes with resolution, including the best fit power-law functions with $\gamma=0.43$ for the LMC and $\gamma=0.24$ for in the SMC. Leroy et al. (2013b) find a best fit $\gamma$ for the scatter in $\log \left(\tau_{\text {dep }}^{\mathrm{mol}}\right)$ in the range of $0-0.8$ with an average of $\gamma=0.5$ (shown by the thick dashed line in Figure 11).

If a galaxy has a fixed $\tau_{\text {dep }}^{\text {mol }}$ and star formation proceeds randomly and independently in separate regions within the resolution element, then behaves like Poisson noise and $\sigma \propto \sqrt{N^{-1}}$, where $N$ is the number of star-forming regions. For a region of size $l, N \propto l^{2}$ so that $\sigma \propto l^{-1}$ or a power-law scaling of $\gamma=1$. Both Feldmann et al. (2011) and Kruijssen \& Longmore (2014) find that the scatter in $\log \left(\tau_{\text {dep }}^{\text {mol }}\right)$ scales with a rough power-law scaling with an index of $\gamma=0.5$ at larger ( 200 pc) scales. Feldmann et al. (2011) expect this shallow scaling as a result of star formation occurring in a $2 \mathrm{D}$ disk galaxy. However, Kruijssen \& Longmore (2014) find similar shallower slopes with uncorrelated, independent star-forming

\footnotetext{
${ }^{15}$ We use $\gamma$ instead of $\beta$ (used in Leroy et al. 2013b) as the variable representing the exponent to avoid confusion with the dust emissivity index $\beta$.
}

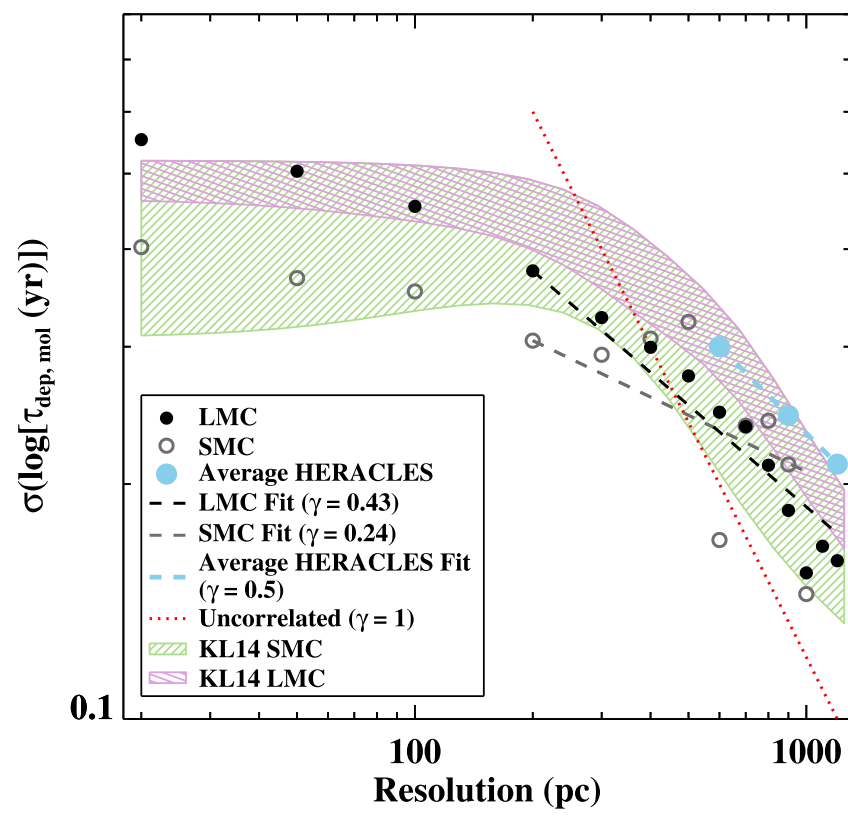

Figure 11. We fit power laws (dashed lines) to the change in scatter in the depletion time as a function of scale for the LMC (black filled circles) and SMC (gray filled circles), and find that the power-law exponent $(\gamma)$ is low, indicating correlation of star formation throughout the galaxies likely due to synchronization by a large-scale process. The thick light blue line shows the line for $\gamma=0.5$, the average fit to the nine HERACLES galaxies that had high enough resolution ( $r \sim 400 \mathrm{pc})$ and the expected scaling for a disk galaxies from simulations by Feldmann et al. (2011). The purple and green hashed lines show the estimates of the scatter due to independent star-forming regions from the Kruijssen \& Longmore (2014) model (KL14) with upper and lower limits for the LMC and SMC, respectively. For comparison, the red dashed line shows how the data would behave if there was no spatial correlation between the star formation and molecular gas at large spatial scales.

regions due to the contribution to the scatter from the time evolution of the star-forming regions and from the underlying distribution of GMC masses. The model from Kruijssen \& Longmore (2014) shows that the scatter due to Poisson noise dominates at large size scales that sample multiple star-forming regions $(>100 \mathrm{pc})$. At small scales, the Poisson noise disappears due to the fact only one star-forming region will be sampled and the scatter from the time evolution of the starforming regions and from the underlying distribution of GMC masses drives the variation in the ratio of star formation rate to molecular gas and $\tau_{\text {dep }}^{\text {mol }}$.

Figure 11 shows the data for the LMC and SMC, the HERACLES galaxies, and the corresponding Kruijssen \& Longmore (2014) model predictions. For the SMC model predictions, we set the upper limit to the upper limit values for the LMC and the lower limit the same as for the LMC but with $t_{\mathrm{gas}}=10 \mathrm{Myr}$ (approximate free-fall time of a GMC) and $\sigma_{\mathrm{MF}}=0.2 \mathrm{dex}(60 \%$ of the scatter in GMC masses in the LMC). We see general agreement between the trend in the observed relationship and the model predictions, however, the LMC data fall below the predicted lower limit (see Section 5.2) at large size scales $(>200 \mathrm{pc})$. The most uncertain parameter in Kruijssen \& Longmore (2014), due to lack of observational constraints, is the scatter due to the time evolution of the gas flux and star formation rate flux $\left(\sigma_{\text {evol,1g }}, \sigma_{\text {evol,1s }}\right)$. Decreasing $\sigma_{\text {evol }}$ further from $0.15 \mathrm{dex}$ to $0.1 \mathrm{dex}$ brings the model predictions much closer to the LMC observations at scales $>100$ pc. 
If we apply the interpretation of Feldmann et al. (2011), the shallower decline in the amount of scatter with increasing averaging scale seen in the LMC (and SMC) could be caused by increased spatial correlation between individual starforming regions. Correlation of star-forming regions, both spatially and temporally, would cause individual star-forming regions to be at similar evolutionary phases throughout large parts of the galaxy and could explain the need for a lower amount of scatter from the time evolution for the gas flux and star formation rate. Large-scale spatial correlation in star formation requires a physical mechanism to synchronize star formation, such as bursts of star formation throughout large parts of the galaxies driven by tidal interactions or ram pressure. The star formation histories of both the LMC and SMC indicated that there have been recent bursts of star formation throughout large parts of the galaxies, likely due to interaction between the galaxies and the Milky Way, and is possibly driving the shorter molecular gas depletion times. The lower amount of scatter in $\log \tau_{\text {dep }}^{\text {mol }}$ at larger size scales observed in the LMC (and more tenuously in the SMC) could also be due to large-scale synchronization of star formation.

\subsection{Comparison to Star Formation Model Predictions}

The Magellanic Clouds provide ideal laboratories to test models of star formation given their low metallicity. Although it is higher metallicity, the geometry of the LMC is better understood than the more irregular SMC. There are few nearby low-mass, low-metallicity systems and measuring their molecular gas content is challenging as they are often weakly emitting in $\mathrm{CO}$ and, when $\mathrm{CO}$ is observed, it is unclear what CO-to- $\mathrm{H}_{2}$ conversion factor should be applied. The dust-based molecular gas estimates for the LMC and SMC allow us to test metallicity-dependent models of star formation at high resolution. We examine the model predictions from Ostriker et al. (2010, hereafter OML10) and Krumholz (2013, hereafter $\mathrm{KMT}+$ ), a recent update of the Krumholz et al. (2009) model modified for atomic-dominated regions. Both models take the total gas surface density $\left(\sum_{\text {gas }}\right)$ and metallicity $\left(Z^{\prime}\right)$ as input parameters and predict the fraction of molecular gas, and from that the star formation rate.

The OML10 model determines the star formation rate based on a balance between vertical gravity in the disk and the pressure of the diffuse ISM, which is controlled by star formation feedback. OML10 relates thermal pressure to $\Sigma_{\mathrm{SFR}}$, whereas Ostriker \& Shetty (2011) relate turbulence to $\Sigma_{\text {SFR }}$. While the star-forming gas in the OML10 model is not strictly molecular gas, but rather bound clouds, we identify our estimate of $\Sigma_{\mathrm{H}_{2}}$ with the model parameter $\Sigma_{\text {gbc }}$, the surface density of gas in gravitationally bound clouds (note that in our methodology both $\mathrm{H}_{2}$ and any optically thick $\mathrm{H}$ i are effectively indistinguishable). This ignores the fact that in very dense regions a significant fraction of the molecular gas could be not self-gravitating, a concern that is probably important in starburst environments but unlikely to matter in the Magellanic Clouds. The reverse concern, that $\mathrm{H}_{\mathrm{I}}$ may make a significant contribution to the cloud bounding mass, is likely a more significant consideration in these sources, although its magnitude is difficult to evaluate. The KMT + model is based on the assumption that the fraction of molecular gas is mainly determined by the balance between the dissociating UV radiation field and the shielding of the gas. The KMT + model adds to Krumholz et al. (2009) the condition that in a region with a low star formation rate, hence a low UV field, the threshold density of the cold neutral medium is no longer set by two-phase equilibrium between the cold and warm neutral medium, but rather by hydrostatic equilibrium.

Both the KMT+ and OML10 models use the mid-plane pressure, which requires an estimate of the density of stars and dark matter in the disk to determine the gravitational pressure. We estimate the stellar surface density by applying the mass-tolight conversion from Leroy et al. (2008) to the $3.6 \mu \mathrm{m}$ Spitzer SAGE images of the LMC and SMC. The $\Sigma_{*}$ is then converted to volume density by assuming a stellar disk thickness of $600 \mathrm{pc}$ for the LMC (van der Marel et al. 2002) and $2 \mathrm{kpc}$ for the SMC (following Bolatto et al. 2011). For the LMC, we use the dark matter density profile from Alves \& Nelson (2000), for the SMC we use the profile from Bekki \& Stanimirović (2009) to estimate the dark matter density as a function of radius from the centers of the galaxies. We find that the combined stellar and dark matter densities have ranges of $0.6-0.1 M_{\odot} \mathrm{pc}^{-3}$, with the higher values concentrated in the stellar bar and an $\sim 10 \%$ dark matter contribution in the LMC, and 0.006-0.1 $M_{\odot} \mathrm{pc}^{-3}$ with an $\sim 20 \%$ dark matter contribution in the SMC.

We adopt most of the fiducial model parameter values as described in OML10 and KMT+. The exception is the depletion time in gravitationally bound clouds, $t_{\mathrm{SF}, \mathrm{gbc}}$, for which OML10 uses 2 Gyr based on the average observed value in nearby galaxies. This value was also applied in the SMC results in Bolatto et al. (2011) since the observed $\tau_{\text {dep }}^{\text {mol was not }}$ much lower than 2 Gyr. In this study we find a wider range of depletion timescales, and a measurable change in $\tau_{\text {dep }}^{\text {mol as a }}$ function of spatial scale (Figure 7). It is important to note that $t_{\mathrm{SF}, \mathrm{gbc}}$ is an input parameter of the OML10 model, obtained from observations rather than theory, and its main impact is to change the relation between $\Sigma_{\mathrm{gbc}}$ and $\Sigma_{\mathrm{SFR}}$ since $\Sigma_{\mathrm{gbc}}=t_{\mathrm{SF}, \mathrm{gbc}} \Sigma_{\mathrm{SFR}}$

The self-regulation in the model operates to make $\Sigma_{\mathrm{SFR}}$ insensitive to the choice of $t_{\mathrm{SF} \text {,gbc }}$ over a wide range of total gas surface densities. The results we show in Figure 12 are computed for $t_{\mathrm{SF}, \mathrm{gbc}}=0.5 \mathrm{Gyr}$, which corresponds approxi-

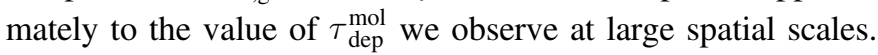
The main effect of changing $t_{\mathrm{SF}, \mathrm{gbc}}$ from 0.5 to $2 \mathrm{Gyr}$ is to slightly lower the predicted star formation rate, particularly at high surface densities $\left(\Sigma_{\text {gas }} \gtrsim 50 M_{\odot} \mathrm{pc}^{-2}\right)$. The robustness of $\Sigma_{\text {SFR }}$ to the choice of $t_{\mathrm{SF}, \text { gbc }}$ in turn means that $\Sigma_{\mathrm{gbc}}$ depends significantly on the value of the depletion timescale. Since we identify $\Sigma_{\mathrm{gbc}}$ with $\Sigma_{\mathrm{H}_{2}}$, the consequence is that the comparison of our measurements of $\Sigma_{\mathrm{H}_{2}}$ with the model predictions are extremely dependent on the assumed $\tau_{\text {dep }}^{\text {mol }}$ and on the

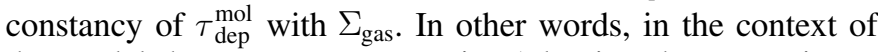
the model they are very uncertain. Adopting the approximate value observed at large spatial scales, $\tau_{\text {dep }}^{\text {mol }} \approx 0.5 \mathrm{Gyr}$, results in a predicted $\Sigma_{\text {gbc }}$ very similar to the observed $\Sigma_{\mathrm{H}_{2}}$.

Figure 12 shows the model predictions for the $\Sigma_{\mathrm{SFR}}$ for the LMC and SMC. Since both models require averaging over the different gas phases, we only compare the predictions to the data at $r \sim 200 \mathrm{pc}$ and $r \sim 1 \mathrm{kpc}$. While the models can predict the molecular-to-atomic ratio (or, diffuse to gravitationally bound for OML10), the models self-regulate in diffuse gas, which is the dominant regime in the LMC and SMC, and predict similar star formation rates. Both KMT+ and OML10 models predict the general trend observed in the relationship between $\Sigma_{\text {gas }}$ and $\Sigma_{\mathrm{SFR}}$. 
The models do not predict the amount of scatter seen in the higher resolution $200 \mathrm{pc}$ data. This is not surprising since both OML10 and KMT+ predict a time-averaged star formation rate and do not recover variations in the star formation rate based on the details on the star formation rate tracer combined with differences in the evolutionary stages of individual star-forming regions. Assuming that the physical interpretation from Feldmann et al. (2011) is correct, which is supported by the predictions of the amount of scatter in $\tau_{\text {dep }}^{\text {mol }}$ at $\sim 100 \mathrm{pc}$ and $1 \mathrm{kpc}$ scales matching our observations, then an important, possibly the dominant, source of scatter is the time-averaging of the star formation rate (over as little as $10 \mathrm{Myr}$, Kennicutt \& Evans 2012) inherent in using $\mathrm{H} \alpha$ and $24 \mu \mathrm{m}$ as star formation rate tracers. We think that it is likely that the under-prediction of the amount of scatter in the OML10/KMT+ model predictions at $200 \mathrm{pc}$ is due to the fact that the star formation models do not include the time-averaging effect of the star formation rate tracer $(\mathrm{H} \alpha)$. The scatter present in the OML10/ $\mathrm{KMT}+$ model predictions come only from the spatial variation in the stellar and dark matter density, which affects the pressure and therefore the predicted amount of star-forming gas. When averaging over larger $(\sim \mathrm{kpc})$ scales, the difference between the scatter in the data and the scatter in the predictions decreases and the two are comparable. The model predictions are most appropriate at large scales where many independent starforming regions are averaged over to account for the fact that star formation is treated as a time-averaged process.

The main differences between the star formation rate from OML10 versus KMT + appear at high $\Sigma_{\text {gas }}$ and low metallicity, where the predictions diverge. All of the KMT+ predictions, independent of metallicity, converge at high $\Sigma_{\text {gas }}$ because once the ISM transitions to $\mathrm{H}_{2}$-rich, which happens past a column density based on the amount of shielding, then the star formation will not behave any differently from high-metallicity galaxies. The OML10 model will tend to continue to predict a lower $\Sigma_{\mathrm{SFR}}$ at high $\Sigma_{\text {gas }}$ because the lower metallicity increases the thermal pressure and reduces the star formation at all surface densities. At the same metallicity and at high $\Sigma_{\text {gas }}$, OML10 will predict lower $\Sigma_{\mathrm{SFR}}$ than KMT+.

\section{SUMMARY AND CONCLUSIONS}

We create $\mathrm{H}_{2}$ maps for the LMC and SMC by using dust emission from HERITAGE Herschel images as a tracer of the total column density of gas and subtracting off the atomic component, which avoids the known biases of using $\mathrm{CO}$ as a tracer of the molecular gas at lower metallicity. Our dust-based methodology has the potential to include optically thick and/or very cold $\mathrm{H}_{\mathrm{I}}$, but we see no evidence of this and assume that all of the gas in our maps is molecular. We find total molecular gas masses of $M_{\mathrm{LMC}}^{\mathrm{mol}}=6.3_{-3.2}^{+6.3} \times 10^{7} \quad M_{\odot} \quad$ and $M_{\mathrm{SMC}}^{\mathrm{mol}} \sim 1.3_{-0.65}^{+1.3} \times 10^{7} M_{\odot}$ including the estimated systematic uncertainty. The structure of the molecular gas maps show good agreement with the structure of the MAGMA ${ }^{12} \mathrm{CO}$ map in the LMC, with the main difference that more extended $\mathrm{H}_{2}$ is seen using our dust-based method.

Using our $\mathrm{H}_{2}$ maps we study the relationship between gas and star formation without relying on a conversion factor to translate $\mathrm{CO}$ emission to the total amount of molecular gas. The high resolution data allow us to study the relationship over 20 to 1000 pc scales. Our main conclusions are as follows:
1. Combining the new molecular gas estimates with the star formation rate from $\mathrm{H} \alpha$ and $24 \mu \mathrm{m}$, we find molecular gas depletion times at $1 \mathrm{kpc}$ scales of $0.4 \mathrm{Gyr}$ for the LMC and 0.6 Gyr for the SMC (Figure 7). These molecular gas depletion times are shorter than the the average found for normal, nearby star-forming galaxies (Figure 5), but are within the scatter found in the STING and HERACLES samples (Rahman et al. 2012; Leroy et al. 2013b; Figure 6). We show that when we include our dust-based molecular gas depletion time measurements with those using CO from the HERACLES sample (Figure 9) we see no trend with metallicity, which suggests that the possible trends seen by (Rahman et al. 2012; Leroy et al. 2013b) could be due to the effect of metallicity on the $\mathrm{CO}-\mathrm{to}-\mathrm{H}_{2}$ conversion factor. The shorter molecular gas depletion times in the LMC and SMC are similar to that observed in M33 (Schruba et al. 2010) and may be associated with the recent bursts in their star formation history.

2. We measure the rank correlation coefficient of the relationships between $\Sigma_{\mathrm{H} \text { I }}$ and $\Sigma_{\mathrm{SFR}}$, and $\Sigma_{\mathrm{H}_{2}}$ and $\Sigma_{\mathrm{SFR}}$ from 20 to $1000 \mathrm{pc}$ size scales (8). The correlation between $\Sigma_{\mathrm{H} \text { I }}$ and $\Sigma_{\mathrm{SFR}}$ is scale independent while the correlation between $\Sigma_{\mathrm{H}_{2}}$ and $\Sigma_{\mathrm{SFR}}$ increases steadily until flattening out at scales of $\sim 200 \mathrm{pc}$ and larger, and on those scales is better correlated than $\Sigma_{\mathrm{H} \text { I }}$ and $\Sigma_{\mathrm{SFR}}$.

3. We measure the scatter in the molecular gas depletion time as a function of size scale (Figure 11). We have compared the observed scatter in the molecular gas depletion time to the predictions from the simulations by Feldmann et al. (2011) and the model by Kruijssen \& Longmore (2014). We find that both can produce the behavior of the scatter with size scale, which suggests that scatter in the $\Sigma_{\mathrm{SFR}}-\Sigma_{\mathrm{H}_{2}}$ relation may be driven largely by the time-averaging effect of the star formation rate tracer combined with instantaneous measurements of the molecular gas at large scales $(>100 \mathrm{pc})$. From comparison with Feldmann et al. (2011) and Kruijssen \& Longmore (2014), we see possible evidence of synchronization of star formation in how the amount of scatter changes with size scale in the LMC (and potentially the SMC), perhaps due to star formation on large scales caused by interactions.

4. We have compared the observed $\Sigma_{\mathrm{SFR}}$ to the predictions from OML10 and KMT+ star formation models (Figure 12) and find wide agreement, indicating that the inclusion of a diffuse neutral medium is important for predicting the star formation rate in atomic-dominated systems like the Magellanic Clouds. Neither model captures the full extent of the scatter seen in the data at $200 \mathrm{pc}$ scales, which we attribute to the time-averaging effect of the star formation rate tracer (as referred to in our previous conclusion).

We thank Mark Krumholz for useful discussions and for use of his Python code implementation of the KMT + model and Diederik Kruijssen for helpful comments and assistance using their code from Kruijssen \& Longmore (2014). We thank Eve Ostriker for very thoughtful comments. A.D.B. and K.E.J. wish to acknowledge partial support from grants NSF-AST0955836 (CAREER), NSF-AST141241, NASA- 

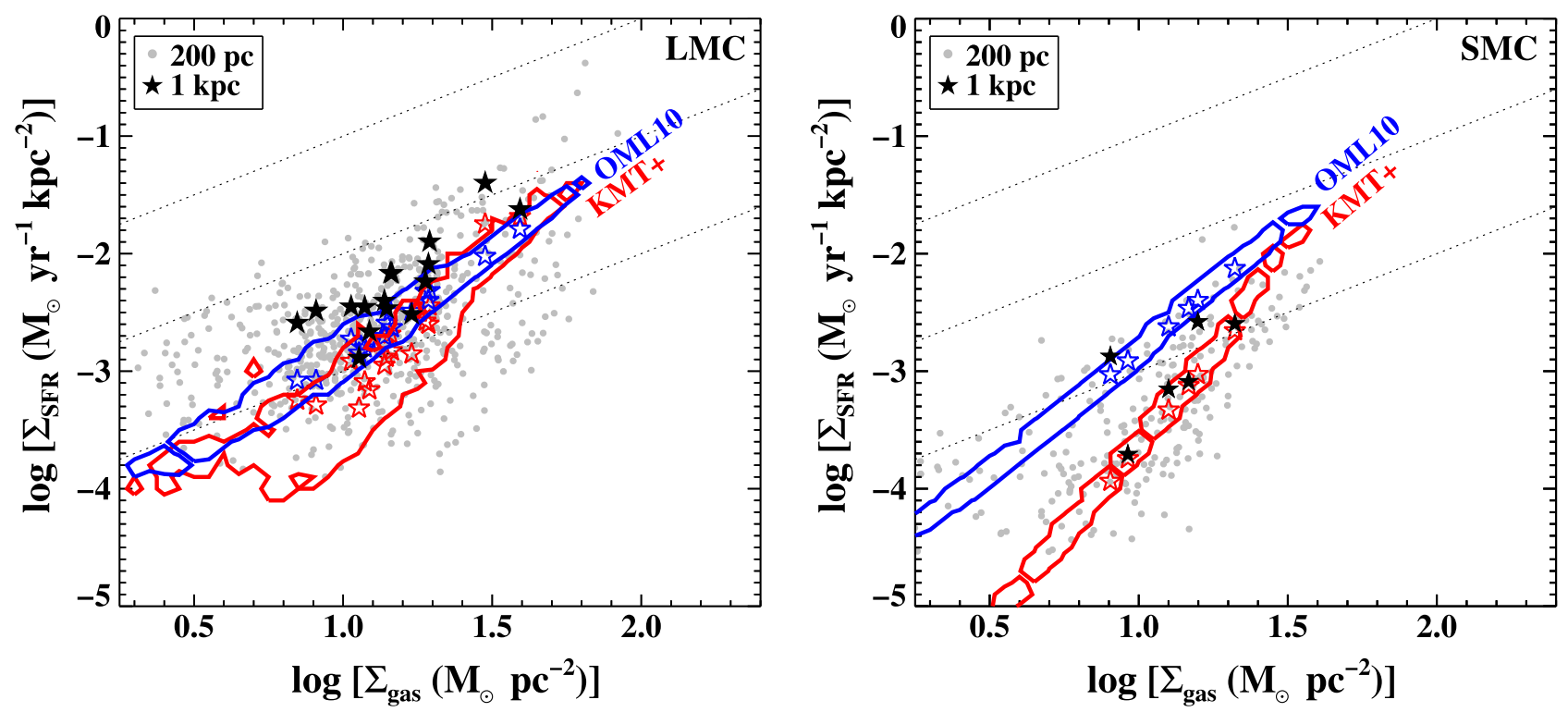

Figure 12. Star formation rate predictions from the OML10 and KMT + models. The gray filled circles show the data at $r=200 \mathrm{pc}$, and the filled stars show the $r=1 \mathrm{kpc}$ data. The diagonal dashed lines indicate constant total gas depletion times (from bottom to top: 10, 1, and $0.1 \mathrm{Gyr}$ ). The contours show the full extent of the distribution of points for the model predictions at $r=200 \mathrm{pc}$ resolution and the open stars show the $r=1 \mathrm{kpc}$ predictions. The KMT+ and OML10 predictions are shown in red and blue, respectively, for the appropriate metallicities for each galaxy and for $t_{\mathrm{SF}, \mathrm{GBC}}=0.5 \mathrm{Gyr}$ for the OML10 model. Both models predict the trend in the data, but do not capture the full extent of the scatter observed.

JPL 1372988, 1483968, and 1454733. We acknowledge financial support from the NASA Herschel Science Center, JPL contracts \#1381522, \#1381650, and \#1350371. We are grateful for the contributions and support from the European Space Agency (ESA), the PACS and SPIRE teams, the Herschel Science Center (especially L. Conversi) and the NASA Herschel Science Center (especially A. Barbar and R. Paladini) and the PACS and SPIRE instrument control centers (especially George Bendo), without whom none of this work would have been possible. M.M. is grateful for support from NSF grant 1312902.

Facilities: Herschel, Spitzer.

\section{APPENDIX A MODELING THERMAL DUST EMISSION}

The dust emission modeling done in this work used a modified blackbody with a fixed emissivity index, $\beta$, to fit the dust temperature $T_{d}$ pixel-by-pixel to the $100,160,250$, and $350 \mu \mathrm{m}$ HERITAGE images. Excluding the $500 \mu \mathrm{m}$ image avoids the issue of possible "excess" dust emission at $\lambda>400 \mu \mathrm{m}$ observed in the SMC and LMC (Planck Collaboration et al. 2011; Gordon et al. 2014). From the fitted $T_{d}$, we calculate $\tau_{160}$ for all $3 \sigma$ fits to $T_{d}$ using

$$
\tau_{160}=\frac{S_{160}\left[\mathrm{MJy} \mathrm{sr}^{-1}\right]}{B_{\nu}\left(T_{\mathrm{dust}}, 160 \mu \mathrm{m}\right)} .
$$

All images are convolved to the resolution of the $350 \mu \mathrm{m}$ data, the lowest resolution $\left(25^{\prime \prime}\right)$, using the kernels from Aniano et al. (2011). We fix $\beta$ in the model to avoid the degeneracy between $T_{d}$ and $\beta$, which can occur when the correlated errors between the Herschel bands are not taken into account. Fixing $\beta$ also follows the previous work in the SMC (Leroy et al. 2009; Bolatto et al. 2011). We adopt $\beta=1.8$ for our fiducial molecular gas map because that is the approximate average value of $\beta_{1}$ found in the BEMBB modeling by Gordon et al. (2014) and similar to $\beta=1.7$ found for M33 using Planck data (F. Israel 2016, private communication). We also create maps using $\beta=1.5$ and $\beta=2.0$ to see how that affects the $\mathrm{H}_{2}$ estimate, since $\beta \sim 1-2$ for carbonaceous grains (Jager et al. 1998) and $\beta \sim 2$ for silicate grains (Coupeaud et al. 2011).

The second map uses the BEMBB dust emission modeling results from Gordon et al. (2014), which uses the same Herschel data, but includes the $500 \mu \mathrm{m}$ image and accounts for correlated uncertainty between the different bands. All images are convolved to the resolution of the $500 \mu \mathrm{m}$ data $\left(35^{\prime \prime}\right)$, and thus lower resolution than our first method of dust modeling. The implementation of the correlated uncertainties in Gordon et al. (2014) eliminates the degeneracy between $T_{d}$ and $\beta$, allowing both to be fit by the models. Gordon et al. (2014) fit three different modified blackbody models to the data: a simple modified blackbody, one that allows two temperatures, and one with a broken emissivity index (fits two $\beta$ values and the break wavelength). We use the surface mass density of dust $\left(\Sigma_{\text {dust }}\right)$ from the broken emissivity model (with $0.8<\beta_{1}<2.5$ ) because it produces the smallest residuals and the gas-to-dust ratio falls within the range allowed by elemental abundances. To be comparable to the dust modeling done in this work, we convert the $\Sigma_{\text {dust }}$ map to $\tau_{160}$ :

$$
\tau_{160}=\kappa_{\text {eff, } 160} \Sigma_{\text {dust }}
$$

where $\kappa_{\text {eff, } 160}=11.6\left(\mathrm{~cm}^{2} \mathrm{~g}^{-1}\right)$, which Gordon et al. (2014) found by calibrating the broken emissivity model to reproduce the diffuse Milky Way spectral energy distribution (Compiègne et al. 2011) with a gas-to-dust ratio of 150 , based on the depletion measurements from Jenkins (2009). 

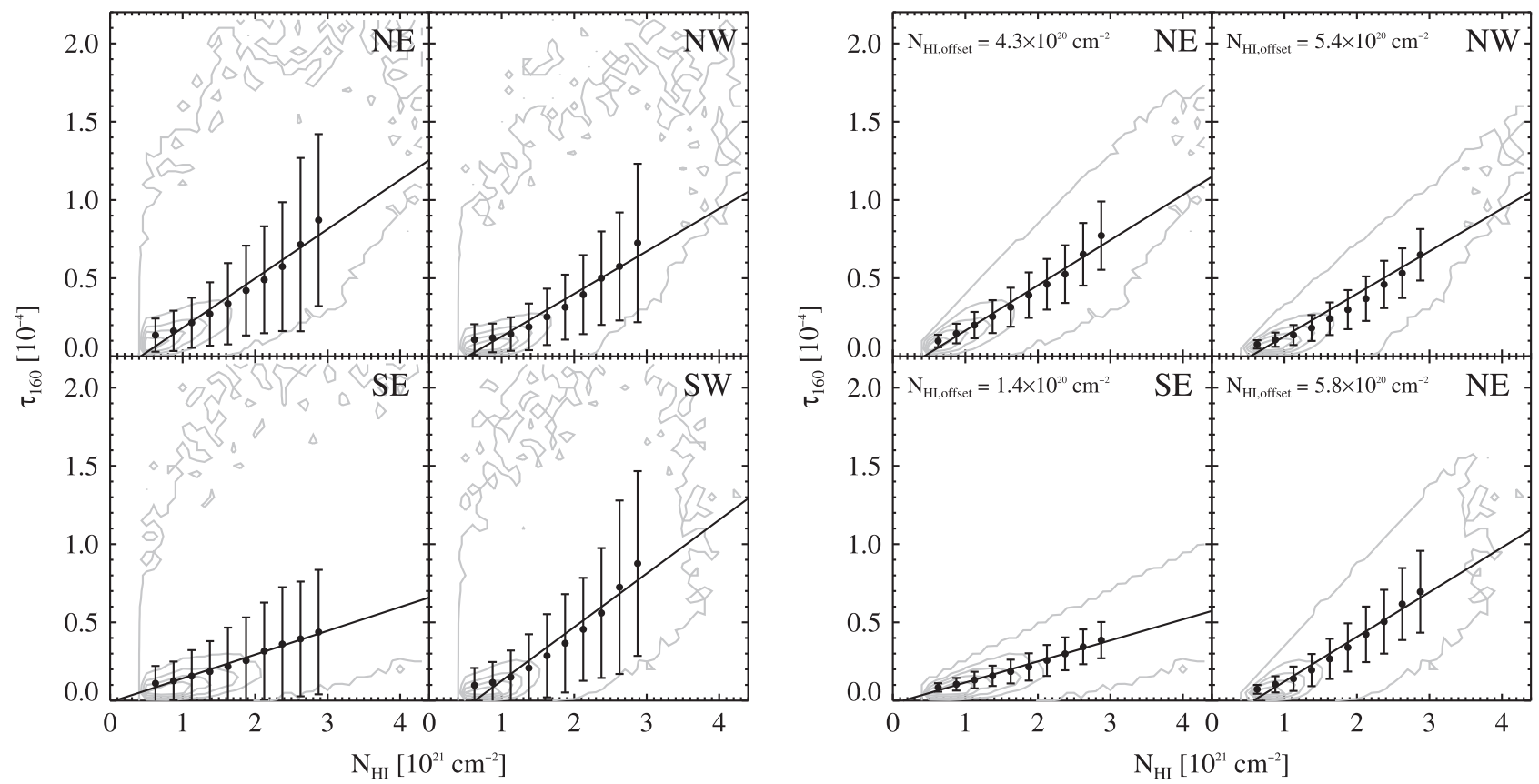

Figure 13. Relationships between $\tau_{160}$ and the column density of $\mathrm{H}_{\mathrm{I}}$ for the quadrants of the LMC using our fiducial $\beta=1.8$ dust modeling results. The contours show the density of points at $80 \%, 60 \%, 40 \%$, and $20 \%$ of the maximum with the outer contour showing the full extent of the distribution. The black points show the median $\tau_{160}$ values with $1 \sigma$ in $2.5 \times 10^{20} \mathrm{~cm}^{-2}$ bins from $1-3 \times 10^{21} \mathrm{~cm}^{-2}$ (chosen to avoid a low number of values and the threshold where CO-dark $\mathrm{H}_{2}$ can exist). The black lines show the fit to the medians, which are used to make rough estimates of the $\mathrm{H}_{2}$ and to determine the $\mathrm{H}$ I offset. The left-hand set of plots has only regions near bright $\mathrm{CO}$ masked. The right-hand set of plots has additional regions with significant estimated $\mathrm{H}_{2}$ masked (see Section 3.1) based on the quadrant fits to the binned median values of the distribution shown in the plots on the left.
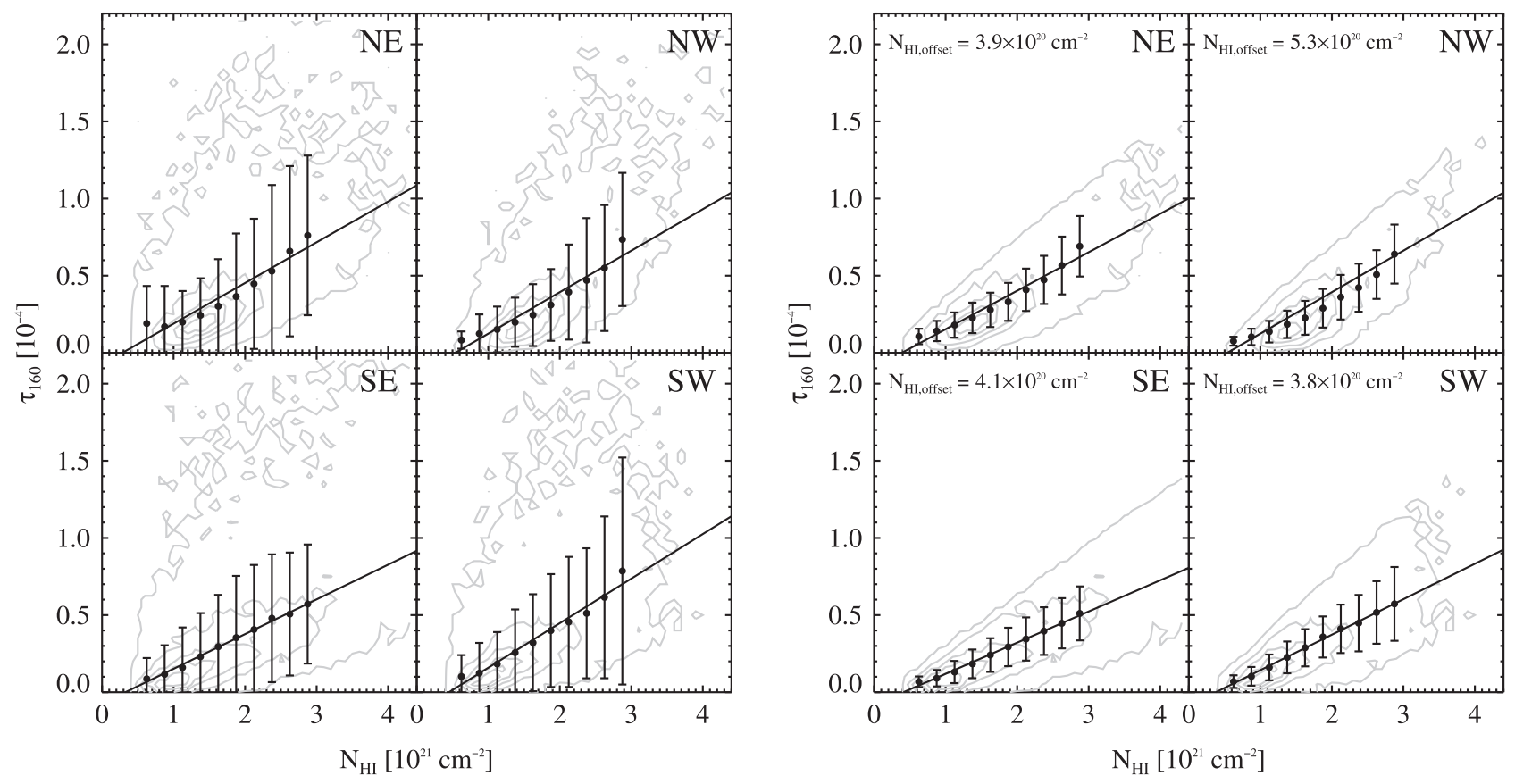

Figure 14. Same as Figure 13 but with $\tau_{160}$ from the Gordon et al. (2014) dust modeling results.

\section{APPENDIX B \\ OFFSETS IN H I VERSUS DUST}

The $\mathrm{H}_{2}$ mapping method assumes that the gas-to-dust ratio in the diffuse, atomic ISM is the same as in the dense, molecular regions. As part of the mapping, we investigated the global and regional relationship between $N_{\mathrm{H}}$ and $\tau_{160}$. The global relationship between $N_{\mathrm{HI}}$ to $\tau_{160}$ is primarily defined by one linear relationship (equivalent to a single dust-to-gas ratio) with a large amount of scatter (see Figure 1). We split the galaxies into quadrants to fit the offset in $\mathrm{H}_{\mathrm{I}}$ (see Figures 13 and 14). For the LMC, we split the south-east quadrant into 16 smaller regions due to the complexity of this part of the galaxy: the Molecular Ridge and an $\mathrm{H}_{\mathrm{I}}$ streamer that extends to become part of the Magellanic Bridge. Figure 15 shows the fitted 

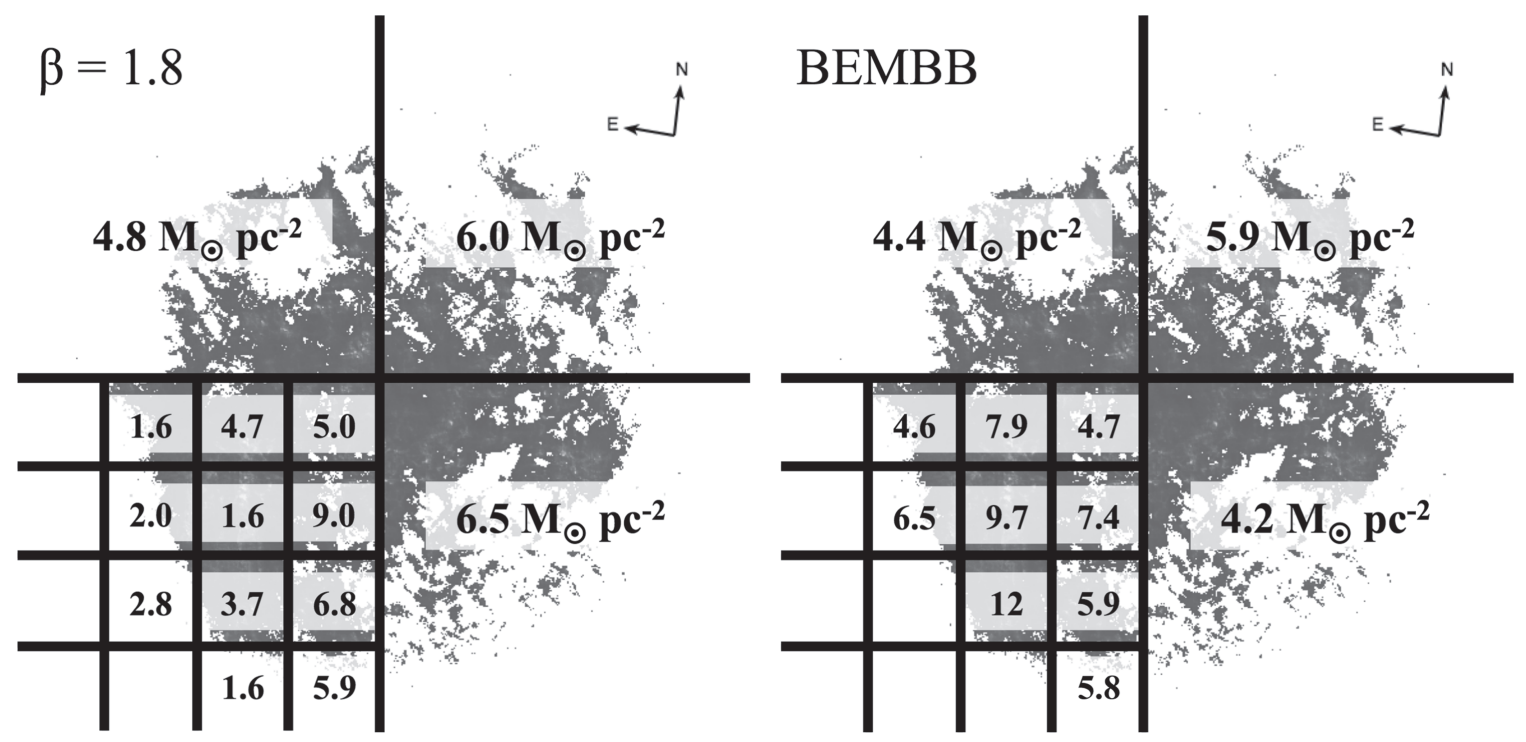

Figure 15. The $\mathrm{H}$ i offset fit for each region in the LMC for the fiducial $\beta=1.8$ dust modeling in this work (left) and Gordon et al. (2014) (right). The background gray-scale shows the $\tau_{160}$ map from the Gordon et al. (2014) modeling.

offsets in $\Sigma_{\mathrm{H} \text { I }}$ in the LMC, with the offsets typically being $\sim 5 M_{\odot} \mathrm{pc}^{-2}$.

By splitting up the LMC into four equal quadrants and looking at the regional relationships between $N_{\mathrm{H} \text { I }}$ and $\tau_{160}$, we found that the offset distribution is coming from the south-west quadrant. We further checked for smaller regional variation within the different molecular gas complexes and found the majority of offset points to be coming from the Molecular Ridge. Possible explanations for an offset at higher $N_{\mathrm{H} \text { I }}$ compared to the dust include issues with the background subtraction in the Herschel data or a constant layer of $\mathrm{H}$ I gas with little to no dust along the line-of sight. We note that variations in the gas-to-dust ratio would only change the slope of the distribution, and not just the offset. An issue with background subtraction seems unlikely since the excess offset appears to be correlated with a physical complex and there is no obvious gradient across the quadrant. A layer of low-dust or dust-free $\mathrm{H}_{\mathrm{I}}$ is possible since there is an $\mathrm{H} \mathrm{I}$ streamer extending out of the galaxy in this area that becomes part of the Magellanic Bridge. It is possible the stripped gas could have little to no dust.

Steps for finding $\mathrm{H}$ i offset:

1. Mask all points that likely have molecular gas given the $\mathrm{CO}$ map (all regions within $2^{\prime}$ of bright $\mathrm{CO}$ emission ( $\left.I_{\mathrm{CO}}>3 \sigma\right)$ detections).

2. First fit a linear equation to the binned medians of the diffuse gas $\left(N_{\mathrm{H}}<3 \times 10^{21} \mathrm{~cm}^{-2}\right)$; the slope represents the effective GDR $\left(\delta_{\mathrm{GDR}}\right)$ and the offset gives the $\mathrm{H}_{\mathrm{I}}$ offset.

3. Use the fitted $\delta_{\mathrm{GDR}}$ to estimate the total gas using the dust map $\left(\Sigma_{\text {gas }}=\delta_{\mathrm{GDR}} \Sigma_{\text {dust }}\right)$, subtract $\Sigma_{\mathrm{HI}}$ to obtain the first iteration estimate of $\mathrm{H}_{2}\left(\Sigma_{\mathrm{mol}}=\left(\delta_{\mathrm{GDR}} \Sigma_{\text {dust }}\right)-\Sigma_{\mathrm{H} \mathrm{I}}\right)$.

4. Mask all points near bright $\mathrm{CO}$ and any points that have estimated $\Sigma_{\text {mol }}>0.5 \Sigma_{\mathrm{H} \text { I }}$

5. Refit a linear equation to the binned medians of the diffuse gas with the new mask.

6. Remove the $\Sigma_{\mathrm{H} \text { I }}$ offset from the second iteration of fitting from the $\mathrm{H}_{\mathrm{I}}$ map and use this subtracted $\mathrm{H}_{\mathrm{I}}$ map in the rest of the analysis.

\section{APPENDIX C \\ DIFFUSE $\mathrm{H} \alpha$}

The $\mathrm{H} \alpha$ images of the Magellanic Clouds clearly show an extended, low-level of emission throughout the galaxies, which traces the ISM component referred to as DIG or the warm ionized medium (WIM). In the Milky Way, the filling factor of the WIM ranges from 0.1 to 0.4 , with evidence that it increases with distance from the mid-plane (Berkhuijsen et al. 2006), and contributes $\sim 10 \%-15 \%$ of the total H II emission (Reynolds 1993). More detailed studies of the WIM in the Milky Way have found that its physical conditions differ from conditions in classical H II regions very widely (Haffner et al. 2009). Early work by Kennicutt \& Hodge (1986) demonstrated that an extended $\mathrm{H} \alpha$ component accounts for $\sim 15 \%$ of the flux in the LMC. Later, Kennicutt et al. (1995) found that the diffuse component of the $\mathrm{H} \alpha$ emission, found by comparing the total flux to the integrated flux from $\mathrm{H}$ II regions, is $\sim 25 \%-35 \%$ in the LMC and $\sim 34 \%-40 \%$ in the SMC. These fractions of diffuse or extended emission are consistent with what is found for Magellanic Irregulars (Kennicutt 1989) and in spirals, including the $20 \%-40 \%$ fraction found in M31 (Walterbos \& Braun 1994). The diffuse $\mathrm{H} \alpha$ component is similar to other star-forming galaxies and a significant fraction of the total emission.

While widespread among galaxies, the origin of the DIG is unclear-is it all escaped $\mathrm{H} \alpha$ photons from star-forming regions or is the gas ionized within the diffuse ISM (Rahman et al. 2011 and references therein)? If the gas is primarily ionized within the diffuse ISM by mechanisms not directly related to star formation and not accounted for in the star formation rate calibration, then including the diffuse emission would overestimate the star formation rate. Pellegrini et al. (2012) studied the optical depth of $\mathrm{H}_{\text {II }}$ regions in the Magellanic Clouds and found that the luminosity of escaped ionizing radiation provides enough power to ionize the diffuse gas, suggesting that the diffuse $\mathrm{H} \alpha$ emission in the Magellanic Clouds could all be escaped radiation from young, massive stars in $\mathrm{H}$ II regions. Even if all of the diffuse $\mathrm{H} \alpha$ emission can 
be attributed to star formation, the appropriateness of including the emission in the star formation rate depends on the details of the star formation rate conversion calibration. The calibration by Calzetti et al. (2007) was performed on scales of 200-600 $\mathrm{pc}$, which likely includes some extended emission. We include the diffuse $\mathrm{H} \alpha$ emission in our analysis and convert the $\mathrm{H} \alpha$ maps to star formation rates, assuming that massive stars are responsible for all of the $\mathrm{H} \alpha$ flux.

\section{APPENDIX D \\ COMPARISON TO PREVIOUS DUST-BASED $\mathrm{H}_{2}$ ESTIMATES}

We compare our dust-based molecular gas estimates to similar estimates in the literature and find that all of the estimates are consistent within the uncertainties given differences in methodology and data. Table 3 provides all of our total molecular gas estimates alongside those from the literature. The early estimates from Israel (1997) for both the LMC and SMC were based on low resolution IRAS data, did not directly model the dust emission (using instead a scaled far-IR surface brightness, $\mu_{\mathrm{FIR}}$, based on the difference in dust temperature relative to a fiducial value), and used one effective gas-to-dust ratio for each galaxy based on a few reference positions. These early estimates are likely high due to the lack of long wavelength data, which causes a bias toward higher dust temperature, and therefore high effective gas-to-dust ratios; regardless, the estimates are still with a factor of $\sim 2$ for the $\mathrm{LMC}$ and $\sim 5$ for the SMC.

Bernard et al. (2008) produced a dust-based estimate of the molecular gas for the LMC using the Spitzer SAGE data and found a total molecular mass of $3.3 \times 10^{8} M_{\odot}$. They chose a single $\delta_{\mathrm{GDR}}$ equal to the value at the lowest $5 \%$ level of the $\delta_{\mathrm{GDR}} \quad$ distribution, $\tau_{160} / N_{\mathrm{H} \mathrm{I}}=8.8 \times 10^{-26} \mathrm{~cm}^{2} \quad$ (or $N_{\mathrm{H}} / \tau_{160}=1.1 \times 10^{25} \mathrm{~cm}^{2}$ ). This value of gas-to-gas ratio is consistent with the average values we find in our maps of the gas-to-dust ratio, but our maps have a wide range of values. Bernard et al. (2008) takes this value of the gas-to-dust ratio and applies it to the entire galaxy. The primary difference that drives the higher molecular gas mass estimate is that Bernard et al. (2008) fits lower dust temperatures (median $T_{\mathrm{d}} \sim 18 \mathrm{~K}$ ) to the Spitzer $160 \mu \mathrm{m}$ and IRIS $100 \mu \mathrm{m}$ data. The dust modeling used in this work includes more IR bands and longer wavelength data and we find a higher average dust temperature of $T_{\mathrm{d}}=23 \mathrm{~K}$. Changing $T_{\mathrm{d}}$ from $18 \mathrm{~K}$ to $23 \mathrm{~K}$ (and holding $I_{160}$ constant) results in a decrease in $\tau_{160}$ of a factor of $\sim 3$. When

Table 3

Comparison of Total Molecular Gas Mass Estimates for the LMC and SMC

\begin{tabular}{|c|c|c|c|c|c|}
\hline & Data & Dust Fitting $^{\mathrm{a}}$ & Method & $M_{\mathrm{mol}}\left[10^{7} M_{\odot}\right]^{\mathrm{b}}$ & $\overline{\text { References }}$ \\
\hline \multicolumn{6}{|c|}{ LMC, this work } \\
\hline 1 & Herschel $100-350 \mu \mathrm{m}$ & $\mathrm{MBB}, \beta=1.8$ & $\delta_{\mathrm{GDR}} \operatorname{map}^{\mathrm{c}}$ & 9.9 & (1) \\
\hline 2 & Herschel $100-500 \mu \mathrm{m}$ & BEMBB, $0.8<\beta<2.5$ & $\delta_{\mathrm{GDR}} \operatorname{map}^{\mathrm{c}}$ & 6.3 & (1) \\
\hline 3 & Herschel $100-350 \mu \mathrm{m}$ & $\mathrm{MBB}, \beta=1.5$ & $\delta_{\mathrm{GDR}} \operatorname{map}^{\mathrm{c}}$ & 6.8 & (1) \\
\hline 4 & Herschel $100-350 \mu \mathrm{m}$ & $\mathrm{MBB}, \beta=2.0$ & $\delta_{\mathrm{GDR}} \operatorname{map}^{\mathrm{c}}$ & 10.1 & (1) \\
\hline 5 & Herschel $100-500 \mu \mathrm{m}$ & BEMBB, $0.8<\beta<2.5$ & $\delta_{\mathrm{GDR}} \operatorname{map}^{\mathrm{c}}$, no $\mathrm{H}_{\mathrm{I}}$ offset & 13.4 & (1) \\
\hline 6 & Herschel $100-500 \mu \mathrm{m}$ & BEMBB, $0.8<\beta<2.5$ & $\mathrm{GDR}=380^{\mathrm{d}}$ & 1.1 & (1) \\
\hline 7 & Herschel $100-500 \mu \mathrm{m}$ & BEMBB, $0.8<\beta<2.5$ & $\mathrm{GDR}=540^{\mathrm{d}}$ & 3.9 & (1) \\
\hline 8 & Herschel $100-350 \mu \mathrm{m}$ & $\mathrm{MBB}, \beta=1.8$ & $\delta_{\mathrm{GDR}} \operatorname{map}^{\mathrm{c}}, \delta_{\mathrm{GDR}, \mathrm{dense}}=0.5 \delta_{\mathrm{GDR}, \mathrm{map}}$ & 4.5 & (1) \\
\hline 9 & Herschel $100-500 \mu \mathrm{m}$ & $\mathrm{BEMBB}, 0.8<\beta<2.5$ & $\delta_{\mathrm{GDR}} \operatorname{map}^{\mathrm{c}}, \delta_{\mathrm{GDR}, \mathrm{dense}}=0.5 \delta_{\mathrm{GDR}, \text { map }}$ & 4.0 & (1) \\
\hline 10 & Herschel $100-500 \mu \mathrm{m}$ & $\mathrm{BEMBB}, 0.8<\beta<2.5$ & $\mathrm{GDR}_{\text {diffuse }}=540, \mathrm{GDR}_{\text {dense }}=330^{\mathrm{d}}$ & 2.2 & (1) \\
\hline \multicolumn{6}{|c|}{ LMC, literature } \\
\hline 11 & $\begin{array}{l}\text { Herschel } 100-500 \mu \mathrm{m}, \\
\text { MAGMA }{ }^{12} \mathrm{CO}\end{array}$ & $\mathrm{BEMBB}, 0.8<\beta<2.5$ & $\mathrm{GDR}_{\text {diffuse }}=380, X_{\mathrm{CO}}=2 \times 10^{20}$ & 2.7 & (2) \\
\hline 12 & IRIS, Spitzer 3-160 $\mu \mathrm{m}$ & Desert et al. (1990) & lowest $5 \% \delta_{\mathrm{GDR}}$ & 33 & (3) \\
\hline 13 & IRAS $12-100 \mu \mathrm{m}$ & $\cdots$ & reference $N_{\mathrm{H}} / \mu_{\mathrm{FIR}}$ & 15.4 & (4) \\
\hline 14 & NANTEN ${ }^{12} \mathrm{CO}$ & $\ldots$ & Galactic $\alpha_{\mathrm{CO}}=4.3$ & 1.25 & (5) \\
\hline 15 & NANTEN ${ }^{12} \mathrm{CO}$ & $\cdots$ & $X_{\mathrm{CO}}=7 \times 10^{20}$ & 5 & (5) \\
\hline \multicolumn{6}{|c|}{ SMC, this work } \\
\hline 16 & Herschel $100-500 \mu \mathrm{m}$ & BEMBB, $0.8<\beta<2.5$ & $\delta_{\mathrm{GDR}} \operatorname{map}^{\mathrm{c}}$ & 2.0 & (1) \\
\hline \multicolumn{6}{|c|}{ SMC, literature } \\
\hline 17 & $\begin{array}{l}\text { Herschel } 100-500 \mu \mathrm{m} \text {, } \\
\text { NANTEN }{ }^{12} \mathrm{CO}\end{array}$ & BEMBB, $0.8<\beta<2.5$ & $\mathrm{GDR}_{\mathrm{diffuse}}=1200, X_{\mathrm{CO}}=1 \times 10^{21}$ & 0.2 & (2) \\
\hline 18 & Spitzer $70-160 \mu \mathrm{m}$ & $\mathrm{MBB}, \beta=1.5$ & $\delta_{\mathrm{GDR}} \operatorname{map}^{\mathrm{c}}$ & 2.2 & (6) \\
\hline 19 & IRIS 100, Spitzer $160 \mu \mathrm{m}$ & Dale \& Helou (2002) & regional GDR & 3.2 & (7) \\
\hline 20 & IRAS $12-100 \mu \mathrm{m}$ & $\cdots$ & reference $N_{\mathrm{H}} / \mu_{\mathrm{FIR}}$ & 10.5 & (4) \\
\hline 21 & NANTEN ${ }^{12} \mathrm{CO}$ & $\cdots$ & Galactic $\alpha_{\mathrm{CO}}=4.3$ & 0.07 & (8) \\
\hline 22 & NANTEN ${ }^{12} \mathrm{CO}$ & $\cdots$ & $X_{\mathrm{CO}}=2.5 \times 10^{21}$ & 0.42 & (8) \\
\hline
\end{tabular}

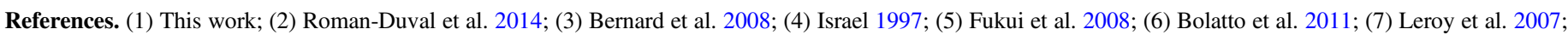
(8) Mizuno et al. 2001.

Notes.

${ }^{\mathrm{a}} \mathrm{MBB}=$ modified blackbody, BEMBB $=$ broken emissivity modified blackbody.

${ }^{\mathrm{b}}$ Assuming $d_{\mathrm{LMC}}=50 \mathrm{kpc}$ and $d_{\mathrm{SMC}}=62 \mathrm{kpc}$.

${ }^{\mathrm{c}}$ Map of spatially varying $\delta_{\mathrm{GDR}}$, see Section 3.1.

${ }^{\mathrm{d}}$ Does not include factor of 1.36 contribution from helium. 
the similar gas-to-dust ratio is applied to the map of higher values of $\tau_{160}$, the total gas, and therefore the molecular gas mass estimate is higher. Scaling our $\tau_{160}$ up by a factor of 3 yields a total molecular gas mass of $2.1 \times 10^{8} M_{\odot}$, which is comparable to the Bernard et al. (2008) estimate. The $M_{\mathrm{mol}}$ estimate from Bernard et al. (2008) can serve as an upper limit to the amount of gas associated with the excess far-IR emission.

The most recent estimates for the molecular gas masses come from Roman-Duval et al. (2014), which also used the dust modeling from Gordon et al. (2014). While this work focuses on creating maps of the molecular gas, Roman-Duval et al. (2014) studies the global relationship between the gas and dust in the different gas phases and does not explore any spatial variations. The molecular gas mass estimate we show in Table 1 combines the estimate of the molecular gas traced by bright $\mathrm{CO}$ emission (using the fiducial $X_{\mathrm{CO}}=2 \times 10^{20} \mathrm{~cm}^{-2}$ $\left(\mathrm{K} \mathrm{km} \mathrm{s}^{-2}\right)^{-1}$ in the LMC and $X_{\mathrm{CO}}=1 \times 10^{21} \mathrm{~cm}^{-2}(\mathrm{~K} \mathrm{~km}$ $\left.\mathrm{s}^{-2}\right)^{-1}$ in the SMC) with the estimate of the amount of molecular gas not traced by bright $\mathrm{CO}$ emission ("CO-dark" or "CO-faint"). They estimate the amount of molecular gas not traced by $\mathrm{CO}$ by applying the diffuse gas-to-dust ratio $\left(\mathrm{GDR}^{\text {dif }}=380\right.$ in the $\mathrm{LMC}$ and $\left.\mathrm{GDR}^{\mathrm{dif}}=1200\right)$ to the regions where molecular gas is expected, based on the dust surface density, but no $\mathrm{CO}$ is detected. The total molecular gas mass estimates for the LMC and SMC are significantly lower than the estimates from this work. Roman-Duval et al. (2014) uses a constant gas-to-dust ratio while we use a map of the gasto-dust ratio, the average gas-to-dust ratios from our maps are $\sim 50 \%$ larger than the average values by Roman-Duval et al. (2014), and they use CO to estimate part of the molecular gas. The main factor driving the lower molecular gas is the low gasto-dust ratio applied uniformly across the galaxies. The difference in gas-to-dust ratio is largely due to the difficulty in fitting a linear relation to a noisy distribution $\left(\Sigma_{\text {gas }}-\Sigma_{\text {dust }}\right)$; Roman-Duval et al. (2014) found a range in the fitted global GDR $^{\text {dif }}$ of 380-540 depending on the fitting method.

The new estimate for the SMC using the Herschel data is lower than the estimates based on Spitzer data: $\sim 40 \%$ lower than the estimate from Bolatto et al. (2011), and $~ 60 \%$ from Leroy et al. (2007). This is well within the factor of 2-3 estimated systematic uncertainty from Bolatto et al. (2011). Given the differences in methodology used in all of the previous estimates and their respective levels of uncertainty, we find all of the molecular gas mass estimates to be consistent.

\section{APPENDIX E IMPLEMENTATION OF KMT+}

Here we explain how the KMT + code was implemented with a brief description of the equations involved in the model. The KMT + model takes the total surface density of gas $\left(\Sigma_{\text {gas }}\right)$, the volume mass density of the stars and dark matter in the disk $\left(\rho_{\mathrm{sd}}\right)$, the metallicity normalized to solar $\left(Z^{\prime}\right)$, and a "clumping parameter" ( $f_{c}$ in KMT,$+ c$ in KMT09) as input parameters and solves for the volume number density of the cold neutral medium $\left(n_{\mathrm{CNM}}\right)$, the fraction of $\mathrm{H}_{2}\left(f_{\mathrm{H}_{2}}\right)$, the strength of the radiation field normalized to the solar value $\left(G_{0}^{\prime}\right)$, and the star formation rate $\left(\dot{\Sigma}_{*}\right)$. The model is based on the following equations (Equations (8), (9), (10), (14), and (15) from Krumholz (2013)). The fraction of $\mathrm{H}_{2}$,
$f_{\mathrm{H}_{2}} \equiv \Sigma_{\mathrm{H}_{2}} /\left(\Sigma_{\mathrm{H}_{\mathrm{I}}}+\Sigma_{\mathrm{H}_{2}}\right)$, is determined by

$$
\begin{gathered}
f_{\mathrm{H}_{2}}= \begin{cases}1-(3 / 4) s /(1+0.25 s), & s<2 \\
0, & s \geqslant 2\end{cases} \\
s \approx \frac{\ln \left(1+0.6 \chi+0.01 \chi^{2}\right)}{0.6 \tau_{c}}, \tau_{c}=0.066 f_{c} Z^{\prime} \Sigma_{0} \\
\chi=7.2 \frac{G_{0}^{\prime}}{n_{1}}, n_{1}=\frac{n_{\mathrm{CNM}}}{10 \mathrm{~cm}^{-3}} .
\end{gathered}
$$

With a value for $f_{\mathrm{H}_{2}}$, we can then calculate the star formation rate per unit area,

$$
\dot{\Sigma}_{*}=f_{\mathrm{H}_{2}} \epsilon_{\mathrm{ff}} \frac{\Sigma}{t_{\mathrm{ff}}} \text { with } t_{\mathrm{ff}} \approx \frac{\pi^{1 / 4}}{\sqrt{8}} \frac{\sigma_{g}}{G\left(\Sigma_{\mathrm{GMC}}^{3} \Sigma\right)^{1 / 4}},
$$

where $\epsilon_{\mathrm{ff}} \approx 0.01, t_{\mathrm{ff}}$ is the free-fall time of the molecular gas, $\sigma_{g}$ $\approx 8 \mathrm{~km} \mathrm{~s}^{-1}$ is the velocity dispersion of the galactic disc and $\Sigma_{\mathrm{GMC}} \approx 85 M_{\odot} \mathrm{pc}^{-2}$ is the characteristic surface density of self-gravitating molecular clouds. At the same time, the radiation field is proportional to the surface density of the star formation rate (following OML10),

$$
G_{0}^{\prime} \approx \frac{\dot{\Sigma}_{*}}{\dot{\Sigma}_{*, 0}}
$$

with the normalization set by the conditions in the solar neighborhood, $\dot{\Sigma}_{*, 0}=2.5 \times 10^{-3} M_{\odot} \mathrm{pc}^{-2} \mathrm{Myr}^{-1}$.

The main ansatz of the KMT + model is that volume density of the cold neutral medium required by hydrostatic equilibrium represents a floor to the possible density, hence the need to calculate both the density from two-phase equilibrium $\left(n_{\mathrm{CNM}, 2 \mathrm{p}}\right)$ and the density from hydrostatic equilibrium $\left(n_{\mathrm{CNM}, \mathrm{hydro}}\right)$ and take the maximum:

$$
n_{\mathrm{CNM}}=\max \left(n_{\mathrm{CNM}, 2 \mathrm{p}}, n_{\mathrm{CNM}, \mathrm{hydro}}\right) .
$$

The density of the CNM in two-phase equilibrium comes from the KMT09 model and depends on the radiation field and the metallicity,

$$
n_{\mathrm{CNM}, 2 \mathrm{p}} \approx 23 G_{0}^{\prime}\left(\frac{1+3.1 Z^{\prime 0.365}}{4.1}\right)^{-1} \mathrm{~cm}^{-2} .
$$

The minimum CNM density from hydrostatic equilibrium requires the thermal pressure $\left(P_{\mathrm{th}}\right)$ and the maximum temperature of the CNM $\left(T_{\mathrm{CNM} \text {,max }}\right)$,

$$
n_{\mathrm{CNM}, \text { hydro }}=\frac{P_{\mathrm{th}}}{1.1 k_{B} T_{\mathrm{CNM}, \max }} .
$$

The maximum temperature the CNM can have and still exist is taken to be $T_{\mathrm{CNM} \text {,max }} \approx 243 \mathrm{~K}$, from the simple analytic model by Wolfire et al. (2003). The thermal pressure equation follows OML10 and calculates the pressure contributions from the gravity contributions from the gas phases, and the stellar and dark matter density,

$$
P_{\mathrm{th}}=\frac{\pi G \Sigma_{\mathrm{HI}}^{2}}{4 \alpha}\left\{1+2 R_{\mathrm{H}_{2}}+\left[\left(1+2 R_{\mathrm{H}_{2}}\right)^{2}+\frac{32 \zeta_{d} \alpha \tilde{f}_{w} c_{w}^{2} \rho_{\mathrm{sd}}}{\pi G \Sigma_{\mathrm{HI}}^{2}}\right]^{1 / 2}\right\}
$$

where $R_{\mathrm{H}_{2}} \equiv \Sigma_{\mathrm{H}_{2}} / \Sigma_{\mathrm{H}_{\mathrm{I}}}=f_{\mathrm{H}_{2}} /\left(1-f_{\mathrm{H}_{2}}\right), \quad \zeta_{d} \approx 0.33$ is a numerical factor whose exact value depends on the shape of 
the gas surface density profile, $\rho_{\mathrm{sd}}$ is the volume density of stars and and dark matter, $c_{w} \approx 8 \mathrm{~km} \mathrm{~s}^{-1}$ is the sound speed in the warm neutral medium, $\tilde{f}_{w}=0.5$ is the adopted fiducial value for the ratio of the mass-weighted mean square thermal velocity dispersion to the square of the warm gas sound speed, and $\alpha \approx$ 5 is the ratio of total pressure in the mid-plane to the thermal pressure $\left(P_{\mathrm{th}}\right)$ due to the additional support provided by turbulence, magnetic fields, and cosmic ray pressure.

To solve these sets of equations, we first guess a value of $G_{0}^{\prime}$ and then a value of $f_{\mathrm{H}_{2}}$ and iterate through both until, first, a self-consistent value of $f_{\mathrm{H}}$ is found (inputting the guess into (14) and checking against the value from (5)). We then evaluate $\dot{\Sigma}_{*}$ using (8) and check our original guess for $G_{0}^{\prime}$ against (9) and iterate until we have self-consistent values for $G_{0}^{\prime}$ and $f_{\mathrm{H}_{2}}$ (within some threshold). For the following predictions using the KMT+ model, we input a map of $\rho_{\text {sd }}$ based on the dark matter profile from rotation curves and stellar density from the Spitzer $3.6 \mu \mathrm{m}$ maps using the conversion from Leroy et al. (2008).

\section{REFERENCES}

Alves, D. R., \& Nelson, C. A. 2000, ApJ, 542, 789

Aniano, G., Draine, B. T., Gordon, K. D., \& Sandstrom, K. 2011, PASP, 123,1218

Bekki, K., \& Stanimirović, S. 2009, MNRAS, 395, 342

Berkhuijsen, E. M., Mitra, D., \& Mueller, P. 2006, AN, 327, 82

Bernard, J.-P., Reach, W. T., Paradis, D., et al. 2008, AJ, 136, 919

Besla, G., Kallivayalil, N., Hernquist, L., et al. 2012, MNRAS, 421, 2109

Bigiel, F., Leroy, A., Walter, F., et al. 2008, AJ, 136, 2846

Bigiel, F., Leroy, A., Walter, F., et al. 2010, AJ, 140, 1194

Bigiel, F., Leroy, A. K., Walter, F., et al. 2011, ApJL, 730, L13

Blanc, G. A., Heiderman, A., Gebhardt, K., Evans, N. J., II, \& Adams, J. 2009, ApJ, 704, 842

Bloemen, J. B. G. M., Deul, E. R., \& Thaddeus, P. 1990, A\&A, 233, 437

Bolatto, A. D., Leroy, A. K., Jameson, K., et al. 2011, ApJ, 741, 12

Bolatto, A. D., Leroy, A. K., Rosolowsky, E., Walter, F., \& Blitz, L. 2008, ApJ, 686, 948

Bolatto, A. D., Simon, J. D., Stanimirović, S., et al. 2007, ApJ, 655, 212

Bolatto, A. D., Wolfire, M., \& Leroy, A. K. 2013, ARA\&A, 51, 207

Bot, C., Boulanger, F., Lagache, G., Cambrésy, L., \& Egret, D. 2004, A\&A, 423, 567

Boulanger, F., Abergel, A., Bernard, J.-P., et al. 1996, A\&A, 312, 256

Braun, R. 2012, ApJ, 749, 87

Bruens, C. 2005, A\&A, 432, 45B

Caldwell, J. A. R., \& Coulson, I. M. 1986, MNRAS, 218, 223

Calzetti, D., Kennicutt, R. C., Engelbracht, C. W., et al. 2007, ApJ, 666, 870

Calzetti, D., Liu, G., \& Koda, J. 2012, ApJ, 752, 98

Calzetti, D., Wu, S.-Y., Hong, S., et al. 2010, ApJ, 714, 1256

Cohen, R. S., Dame, T. M., Garay, G., et al. 1988, ApJL, 331, L95

Compiègne, M., Verstraete, L., Jones, A., et al. 2011, A\&A, 525, AA103

Cormier, D., Madden, S. C., Lebouteiller, V., et al. 2014, A\&A, 564, A121

Coupeaud, A., Demyk, K., Meny, C., et al. 2011, A\&A, 535, A124

Cox, P., Kruegel, E., \& Mezger, P. G. 1986, A\&A, 155, 380

Dale, D. A., \& Helou, G. 2002, ApJ, 576, 159

Dame, T. M., Hartmann, D., \& Thaddeus, P. 2001, ApJ, 547, 792

Davidge, T. J., \& Puzia, T. H. 2011, ApJ, 738, 144

Desert, F.-X., Boulanger, F., \& Puget, J. L. 1990, A\&A, 237, 215

Dickey, J. M., Mebold, U., Marx, M., et al. 1994, A\&A, 289, 357

Dickey, J. M., Mebold, U., Stanimirovic, S., \& Staveley-Smith, L. 2000, ApJ, 536, 756

Draine, B. T., \& Li, A. 2007, ApJ, 657, 810

Dufour, R. J. 1984, in IAU Symp. 108, Structure and Evolution of the Magellanic Clouds, ed. S. van den Bergh, \& K. S. D. de Boer (Dordrecht: Reidel), 353

Dupac, X., Bernard, J.-P., Boudet, N., et al. 2003, A\&A, 404, L11 Feldmann, R., Gnedin, N. Y., \& Kravtsov, A. V. 2011, ApJ, 732, 115 Fukui, Y., \& Kawamura, A. 2010, ARA\&A, 48, 547

Fukui, Y., Kawamura, A., Minamidani, T., et al. 2008, ApJS, 178, 56

Fukui, Y., Kawamura, A., Wong, T., et al. 2009, ApJ, 705, 144

Fukui, Y., Torii, K., Onishi, T., et al. 2015, ApJ, 798, 6
Galametz, M., Hony, S., Galliano, F., et al. 2013, MNRAS, 431, 1596 Galliano, F., Hony, S., Bernard, J.-P., et al. 2011, A\&A, 536, A88

Gao, Y., \& Solomon, P. M. 2004, ApJ, 606, 271

Gaustad, J. E., McCullough, P. R., Rosing, W., \& Van Buren, D. 2001, PASP, 113,1326

Genzel, R., Tacconi, L. J., Combes, F., et al. 2012, ApJ, 746, 69

Glover, S. C. O., \& Mac Low, M.-M. 2011, MNRAS, 412, 337

Gordon, K. D., Meixner, M., Meade, M. R., et al. 2011, AJ, 142, 102

Gordon, K. D., Roman-Duval, J., Bot, C., et al. 2014, ApJ, 797, 85

Grenier, I. A., Casandjian, J.-M., \& Terrier, R. 2005, Sci, 307, 1292

Groenewegen, M. A. T. 2000, A\&A, 363, 901

Haffner, L. M., Dettmar, R.-J., Beckman, J. E., et al. 2009, RvMP, 81, 969

Harris, J., \& Zaritsky, D. 2004, AJ, 127, 1531

Harris, J., \& Zaritsky, D. 2009, AJ, 138, 1243

Haschke, R., Grebel, E. K., \& Duffau, S. 2012, AJ, 144, 107

Heyer, M., Krawczyk, C., Duval, J., \& Jackson, J. M. 2009, ApJ, 699 1092

Hony, S., Gouliermis, D. A., Galliano, F., et al. 2015, MNRAS, 448, 1847

Hopkins, P. F., Kereš, D., Oñorbe, J., et al. 2014, MNRAS, 445, 581

Hughes, A., Meidt, S. E., Colombo, D., et al. 2013, ApJ, 779, 46

Hughes, A., Wong, T., Ott, J., et al. 2010, MNRAS, 406, 2065

Israel, F. P. 1997, A\&A, 328, 471

Israel, F. P., Johansson, L. E. B., Lequeux, J., et al. 1993, A\&A, 276, 25

Israel, F. P., Wall, W. F., Raban, D., et al. 2010, A\&A, 519, A67

Jager, C., Mutschke, H., \& Henning, T. 1998, A\&A, 332, 291

Jenkins, E. B. 2009, ApJ, 700, 1299

Kauffmann, G., Heckman, T. M., White, S. D. M., et al. 2003, MNRAS, 341,54

Kaufman, M. J., Wolfire, M. G., Hollenbach, D. J., \& Luhman, M. L. 1999, ApJ, 527, 795

Kawamura, A., Mizuno, Y., Minamidani, T., et al. 2009, ApJS, 184, 1

Keller, S. C., \& Wood, P. R. 2006, ApJ, 642, 834

Kennicutt, R. C., \& Evans, N. J. 2012, ARA\&A, 50, 531

Kennicutt, R. C., Jr. 1989, ApJ, 344, 685

Kennicutt, R. C., Jr. 1998, ApJ, 498, 541

Kennicutt, R. C., Jr., Bresolin, F., Bomans, D. J., Bothun, G. D., \& Thompson, I. B. 1995, AJ, 109, 594

Kennicutt, R. C., Jr., Calzetti, D., Walter, F., et al. 2007, ApJ, 671, 333

Kennicutt, R. C., Jr., Hao, C.-N., Calzetti, D., et al. 2009, ApJ, 703, 1672

Kennicutt, R. C., Jr., \& Hodge, P. W. 1986, ApJ, 306, 130

Kim, S., Staveley-Smith, L., Dopita, M. A., et al. 1998, ApJ, 503, 674

Kim, S., Staveley-Smith, L., Dopita, M. A., et al. 2003, ApJS, 148, 473

Kruijssen, J. M. D., \& Longmore, S. N. 2014, MNRAS, 439, 3239

Krumholz, M. R. 2012, ApJ, 759, 9

Krumholz, M. R. 2013, MNRAS, 436, 2747

Krumholz, M. R., Dekel, A., \& McKee, C. F. 2012, ApJ, 745, 69

Krumholz, M. R., Leroy, A. K., \& McKee, C. F. 2011, ApJ, 731, 25

Krumholz, M. R., McKee, C. F., \& Tumlinson, J. 2009, ApJ, 699, 850

Kurt, C. M., Dufour, R. J., Garnett, D. R., et al. 1999, ApJ, 518, 246

Lada, C. J., Forbrich, J., Lombardi, M., \& Alves, J. F. 2012, ApJ, 745, 190

Lada, C. J., Lombardi, M., \& Alves, J. F. 2009, ApJ, 703, 52

Lada, C. J., Lombardi, M., \& Alves, J. F. 2010, ApJ, 724, 687

Lada, C. J., Lombardi, M., Roman-Zuniga, C., Forbrich, J., \& Alves, J. F. 2013, ApJ, 778, 133

Lee, C., Leroy, A. K., Schnee, S., et al. 2015a, MNRAS, 450, 2708

Lee, M.-Y., Stanimirović, S., Murray, C. E., Heiles, C., \& Miller, J. 2015b, ApJ, 809, 56

Leroy, A., Bolatto, A., Stanimirović, S., et al. 2007, ApJ, 658, 1027

Leroy, A. K., Bolatto, A., Bot, C., et al. 2009, ApJ, 702, 352

Leroy, A. K., Bolatto, A., Gordon, K., et al. 2011, ApJ, 737, 12

Leroy, A. K., Lee, C., Schruba, A., et al. 2013a, ApJL, 769, L12

Leroy, A. K., Walter, F., Brinks, E., et al. 2008, AJ, 136, 2782

Leroy, A. K., Walter, F., Sandstrom, K., et al. 2013b, AJ, 146, 19

Marx-Zimmer, M., Herbstmeier, U., Dickey, J. M., et al. 2000, A\&A, 354, 787

McKee, C. F., Parravano, A., \& Hollenbach, D. J. 2015, ApJ, 814, 13

Meixner, M., Gordon, K. D., Indebetouw, R., et al. 2006, AJ, 132, 2268

Meixner, M., Panuzzo, P., Roman-Duval, J., et al. 2013, AJ, 146, 62

Mizuno, N., Yamaguchi, R., Mizuno, A., et al. 2001, PASJ, 53, 971

Muller, E., Ott, J., Hughes, A., et al. 2010, ApJ, 712, 1248

Onodera, S., Kuno, N., Tosaki, T., et al. 2010, ApJL, 722, L127

Ostriker, E. C., McKee, C. F., \& Leroy, A. K. 2010, ApJ, 721, 975

Ostriker, E. C., \& Shetty, R. 2011, ApJ, 731, 41

Padoan, P., Kim, S., Goodman, A., \& Staveley-Smith, L. 2001, ApJL, 555, L33

Pagel, B. E. J. 2003, in ASP Conf. Ser. 304, CNO in the Universe, ed. C. Charbonnel, D. Shaerer, \& G. Meynet (San Francisco, CA: ASP), 187 
Pak, S., Jaffe, D. T., van Dishoeck, E. F., Johansson, L. E. B., \& Booth, R. S. 1998, ApJ, 498, 735

Pellegrini, E. W., Oey, M. S., Winkler, P. F., et al. 2012, ApJ, 755, 40

Planck Collaboration, Abergel, A., Ade, P. A. R., et al. 2011, A\&A, 536, A24

Planck Collaboration, Abergel, A., Ade, P. A. R., et al. 2014, A\&A, 566, A55

Planck Collaboration, Ade, P. A. R., Aghanim, N., et al. 2011, A\&A, 536, A17

Rahman, N., Bolatto, A. D., Wong, T., et al. 2011, ApJ, 730, 72

Rahman, N., Bolatto, A. D., Xue, R., et al. 2012, ApJ, 745, 183

Reynolds, R. J. 1993, in ASP Conf. Ser. 35, Massive Stars: Their Lives in the Interstellar Medium, ed. J. P. Cassinelli \& E. B. Churchwell (San Francisco, CA: ASP), 338

Roman-Duval, J., Gordon, K. D., Meixner, M., et al. 2014, ApJ, 797, 86

Roman-Duval, J., Israel, F. P., Bolatto, A., et al. 2010, A\&A, 518, L74

Roychowdhury, S., Huang, M.-L., Kauffmann, G., Wang, J., \& Chengalur, J. N. 2015, MNRAS, 449, 3700

Rubio, M., Garay, G., Montani, J., \& Thaddeus, P. 1991, ApJ, 368, 173

Rubio, M., Lequeux, J., \& Boulanger, F. 1993, A\&A, 271, 9

Russell, S. C., \& Dopita, M. A. 1992, ApJ, 384, 508

Saintonge, A., Kauffmann, G., Wang, J., et al. 2011, MNRAS, 415, 61

Schlafly, E. F., \& Finkbeiner, D. P. 2011, ApJ, 737, 103

Schmidt, M. 1959, ApJ, 129, 243

Schruba, A., Leroy, A. K., Walter, F., Sandstrom, K., \& Rosolowsky, E. 2010, ApJ, 722, 1699

Schruba, A., Leroy, A. K., Walter, F., et al. 2011, AJ, 142, 37

Scowcroft, V., Freedman, W. L., Madore, B. F., et al. 2016, ApJ, 816, 49

Shetty, R., Glover, S. C., Dullemond, C. P., et al. 2011, MNRAS, 415, 3253

Shetty, R., Kauffmann, J., Schnee, S., \& Goodman, A. A. 2009, ApJ, 696, 676
Skibba, R. A., Engelbracht, C. W., Aniano, G., et al. 2012, ApJ, 761, 42 Smith, R. C. \& MCELS Team 1999, in Proc. IAU Symp. 190, New Views of the Magellanic Clouds, ed. Y.-H. Chu et al. (San Francisco, CA: ASP), 28

Stanimirović, S., Staveley-Smith, L., Dickey, J. M., Sault, R. J., \& Snowden, S. L. 1999, MNRAS, 302, 417

Stanimirović, S., Staveley-Smith, L., \& Jones, P. A. 2004, ApJ, 604, 176

Staveley-Smith, L., Kim, S., Calabretta, M. R., Haynes, R. F., \& Kesteven, M. J. 2003, MNRAS, 339, 87

Tacconi, L. J., Genzel, R., Neri, R., et al. 2010, Nature, 463, 781

Tielens, A. G. G. M., \& Hollenbach, D. 1985, ApJ, 291, 722

van der Marel, R. P., Alves, D. R., Hardy, E., \& Suntzeff, N. B. 2002, AJ, 124, 2639

van der Marel, R. P., \& Cioni, M.-R. L. 2001, AJ, 122, 1807

van der Marel, R. P., \& Kallivayalil, N. 2014, ApJ, 781, 121

Verley, S., Corbelli, E., Giovanardi, C., \& Hunt, L. K. 2010, A\&A, 510, A64

Walter, F., Brinks, E., de Blok, W. J. G., et al. 2008, AJ, 136, 2563

Walterbos, R. A. M., \& Braun, R. 1994, ApJ, 431, 156

Wang, J., Heckman, T. M., \& Lehnert, M. D. 1998, ApJ, 509, 93

Wolfire, M. G., Hollenbach, D., \& McKee, C. F. 2010, ApJ, 716, 1191

Wolfire, M. G., Hollenbach, D., \& Tielens, A. G. G. M. 1989, ApJ, 344,770

Wolfire, M. G., McKee, C. F., Hollenbach, D., \& Tielens, A. G. G. M. 2003, ApJ, 587, 278

Wong, T., Hughes, A., Fukui, Y., et al. 2009, ApJ, 696, 370

Wong, T., Hughes, A., Ott, J., et al. 2011, ApJS, 197, 16

Zibetti, S., Charlot, S., \& Rix, H.-W. 2009, MNRAS, 400, 1181 